\title{
Remote Detection of Sodium and Potassium Atomic Emission Signatures as an Indicator of Liquid Carry- Over into Flare Systems
}

\author{
by \\ Zachary R. Milani \\ A thesis submitted to The Faculty of Graduate Studies and Research \\ in partial fulfilment of the degree requirements of \\ Master of Applied Science in Mechanical Engineering \\ Ottawa-Carleton Institute for \\ Mechanical and Aerospace Engineering \\ Department of Mechanical and Aerospace Engineering \\ Carleton University \\ Ottawa, Ontario, Canada \\ June 2021
}

Copyright (C) 2021 - Zachary R. Milani 
The undersigned recommend to

the Faculty of Graduate Studies and Research

acceptance of the thesis

\title{
Remote Detection of Sodium and Potassium Atomic Emission Signatures as an Indicator of Liquid Carry-Over into Flare Systems
}

\author{
Submitted by Zachary R. Milani \\ in partial fulfilment of the requirements for the degree of \\ Master of Applied Science in Mechanical Engineering
}

Dr. Matthew Johnson, Thesis Supervisor

Dr. Ron Miller, Chair, Department of Mechanical and Aerospace Engineering

Carleton University

2021 


\begin{abstract}
Carry-over of salt-laden aerosols into flares at upstream oil and gas production sites is a potentially important driver of adverse flare emissions. However, the frequency of this occurrence at upstream production sites is unknown. Sodium $(\mathrm{Na})$ and potassium $(\mathrm{K})$ are abundant in produced water samples and have persistent atomic emission signatures at $588 / 589$ and 766/769 nm. A pair of spectrometer systems was developed to detect these signatures in flare flames as proof of liquid carry-over. Field measurements of 95 flares at upstream oil and gas production sites in North Dakota, Saskatchewan, and Ecuador found $72 \%$ had detectable $\mathrm{Na}$ or $\mathrm{K}$ signatures, suggesting liquid carry-over is surprisingly common. Analysis of the 76 North Dakota flares found that liquid carry-over was statistically correlated with younger wells and increased volumes of produced oil, water, gas and reported flared/vented volumes. These findings have importance implications for the likely severity of global flare emissions.
\end{abstract}




\section{Acknowledgments}

To Dr. Johnson, I am not sure these two simple words express how truly grateful I am for the countless opportunities you've provided me; the lessons you've taught me; and the travel experiences we've shared these last few years: Thank you. Through extreme patience and unconditional support, you have helped me navigate all of the challenges I faced as a student. I strive to communicate complex ideas using practical, real-world examples as well as you do. It is a pleasure to work with you and to learn from you.

To Dr. Bradley Conrad: Thank you for always welcoming my questions regardless of what you had on your plate. You were always able to explain the nuances of abstract ideas, and to walk me through problems I couldn't quite figure out. Perhaps most important is that you've taught me to identify and pursue the aspects of difficult problems that matter most. Without your time and effort, I would still be trying to figure out that convolution.

To all of the EERL family, past and present: Brian, Dave, Darcy, Carol, Simon, Melina, Josh, Brigid, Jay, Scott, Nick B., Nick H., Parvin, Damon, Cameron, Alex, Ellen, Fraser, Milad, and the FlareNet family: Thank you for all of the good times in and out of the lab. I will forever remember the brief morning talks in Canal, the barbeque we certainly never used, and that time the power went out at $11 \mathrm{am}$.

To Mom, Dad, Alexis, and my grandparents: Thank you. I am lucky to call you my family. Your unconditional love and support made me feel as though I was never more than five minutes away. Coming home to escape it all was always the best medicine, and when I had to leave, your care packages were plenty. "Thanks, Team".

To Cierra, Thank you for everything. I simply couldn't have done this without you. You have shown me what it means to absolutely care and support someone. That said, I am not sure that I ever packed my own suitcase or snacks. And I always had the best snacks. You never let me give up on myself, and were always there when I just needed a break or to laugh. I am truly thankful that you are in my life and by my side. 


\section{Table of Contents}

List of Tables .............................................................................................................................. viii

List of Figures............................................................................................................................... $\mathrm{x}$

Nomenclature …......................................................................................................................... xiv

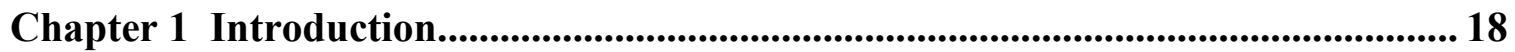

1.1 Gas Flaring and Entrainment of Aerosols into Flare Systems .......................... 18

1.1.1 Flaring in the Upstream Oil and Gas Sector ........................................... 18

1.1.2 Separation of Produced Liquids from Flare Gas at Upstream Oil and Gas

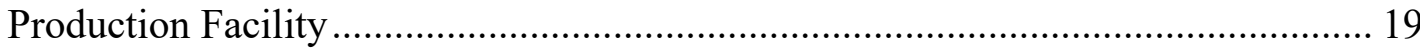

1.1.3 The Formation Processes of Liquid Droplets in the Production Stream at Upstream Oil and Gas Facilities ......................................................................... 20

1.2 Potential Effects of Produced Water Aerosols in Flare Flames ........................... 21

1.3 Proposed Method of Confirming the Presence of Non-Hydrocarbon Liquid in Flare Flames via Atomic Emission Spectroscopy ………………………………..... 23

1.4 Thesis Objectives and Outline.................................................................... 23

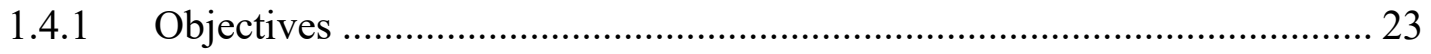

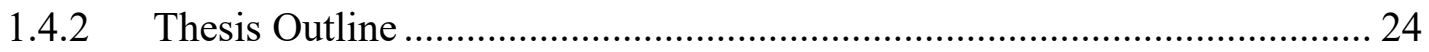

\section{Chapter 2 Review of Atomic Emission Spectroscopy as Applicable to a Remote}

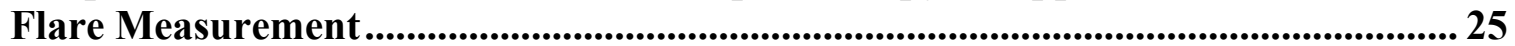

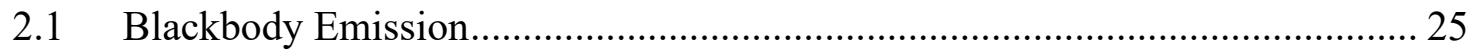

2.1.1 Calculating Instantaneous Gas Kinetic Temperature from Soot Spectra using Radiative Transfer Theory .................................................................... 26

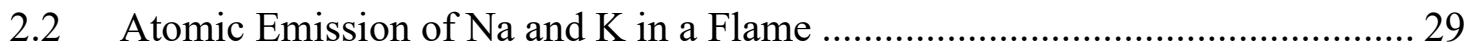

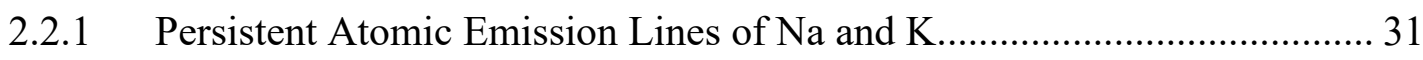

2.3 Local Thermodynamic Equilibrium and Electron Temperature ........................ 32

2.4 Modelling Atomic Emission Considering Self-Absorption and Induced

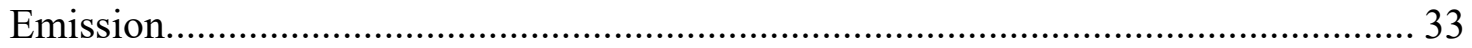

2.5 Molecular Absorption Considering Induced Emission ...................................... 38

Chapter 3 Experimental Setup and Calibrations ............................................................. 41

3.1 The Spectrometer Systems and their Components............................................ 41 
3.1.1 Operation of the Slit Spectrometer ............................................................ 42

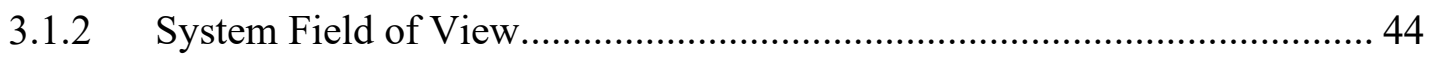

3.1.3 Fine Focusing of the Spectrometer's Entrance Slit to the Focal Point of

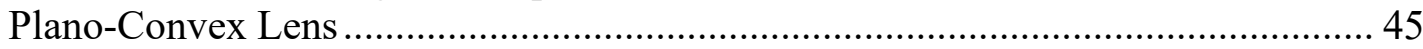

3.2 Relative Spectral Response Calibrations...................................................... 46

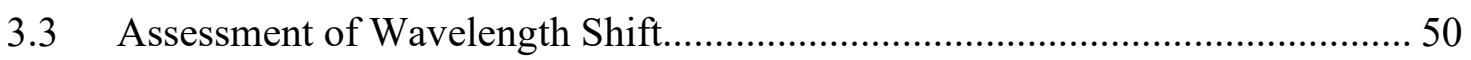

3.3.1 Calculating Wavelength Shift Intervals ..................................................... 52

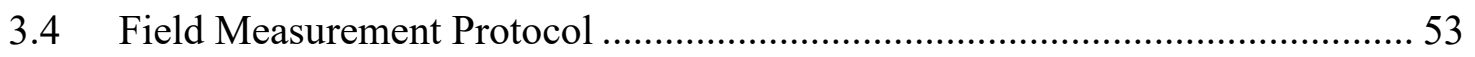

3.5 Experimental Estimation of the Detectability of the Na and K First Resonance

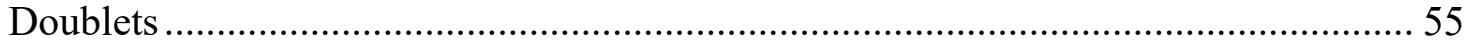

3.5.1 Sensitivity Measurements ......................................................................... 57

Chapter 4 Data Processing ..................................................................................................... 61

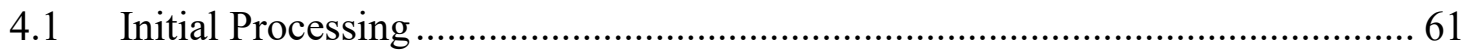

4.1.1 Subtracting the Noise from, and Cropping the Raw Flare Spectra.............. 61

4.1.2 Applying the Relative Spectral Response Calibration Curves ................... 62

4.1.3 Transformation from the Wavelength to Wavenumber Domain ................ 64

4.2 Determining the Effective Temperature of a Flare Flame ................................. 66

4.2.1 Fitting the Soot Temperature Models to Soot Spectra and Calculating the

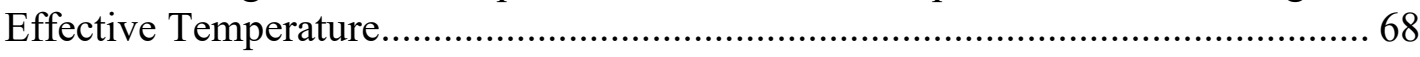

4.3 Modeling Potential Emission Signatures of the Na First Resonance Doublet... 71

4.3.1 Isolating Potential Na Emission Signatures and Fitting the Atomic Emission

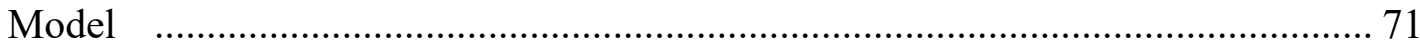

4.3.2 Isolating a Potential Emission Signature of K and Fitting the Atomic

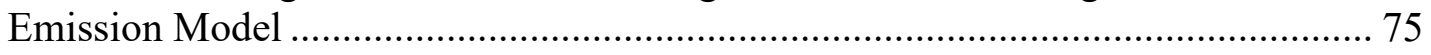

4.4 Peak Identification Metrics ..................................................................... 79

4.4.1 The Signal to Noise Ratio Peak Identification Metric ................................ 79

4.4.2 The Integrated Intensity Ratio Peak Identification Metric ......................... 80

4.4.3 The F test Peak Identification Metric........................................................ 83

4.4.4 Identifying Successful Vs. Null Detections via the Peak Identification

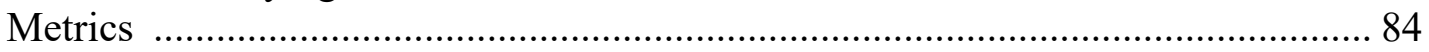

Chapter 5 Results and Discussion .................................................................................... 87

5.1 The Prevalence of Liquid Carry-Over Amongst the Flares Measured in North

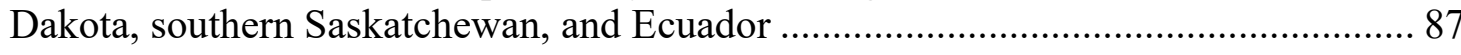

5.2 Flame Temperature Comparison for Flares with/without Detections ................. 92 
5.3 Analysing Correlations Between Production Parameters and Flares with Apparent Liquid Carry-Over in North Dakota .............................................................. 93

5.3.1 Correlations of Species Detections with Well Age..................................... 95

5.3.2 Correlations of Species Detections with Produced Water or Oil Volumes 97

5.3.3 Correlations of Species Detections with Produced Gas or Flared / Vented Volumes

5.4 Relative Importance of Production Parameters in Predicting Liquid Carry-Over in North Dakota Flares 100

5.4.1 Logistic Regression Model for Predicting Species Detection ................... 101

5.4.2 Assessing the Correlations Among Predictor Variables ............................ 101

5.4.3 Comparing the Precision and Accuracy of Logistic Regression Models when Classifying the Species Detection Status of North Dakota Flares ................ 103

5.4.4 Summarizing the Results from the Correlation and Classifier Analyses.. 107

Chapter 6 Conclusion ........................................................................................................ 109

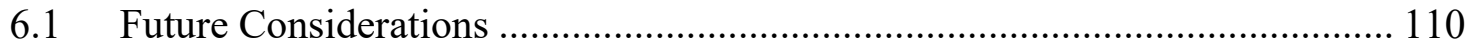

References ...................................................................................................................... 112

Appendix A : Supplemental Derivations and Equations for the Atomic Emission, Molecular Absorption, and Soot Temperature Models................................................. 119

A.1 The Radiative Transfer Equation Derivation for Soot Emission from a Flare Flame Measured by the Spectrometer System......................................................... 119

A.2 The Gaussian Function with Standard Deviation Replaced by Full-Width Half-

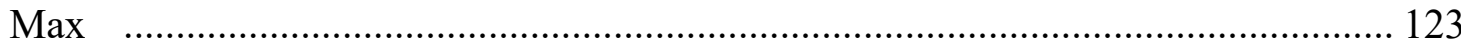

A.3 The Spectral Absorption Coefficient Function used to Model Absorption and

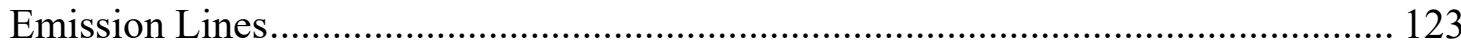

Appendix B : Age Distribution of Active Oil and Gas Wells in North Dakota ........ 125 


\section{List of Tables}

Table 1.1: Important mechanisms for aerosl formation and their estimated droplet diameters (Wines and Brown 1994). 21

Table 2.1: The values of the general variables found in Eq. (2.2) ................................ 27

Table 2.2: Boiling point temperatures for $\mathrm{Na}, \mathrm{NaCl}, \mathrm{K}, \mathrm{KCl}$ (Lewis 2004)................... 29

Table 2.3: Location of the first resonance doublet of $\mathrm{Na}$ and $\mathrm{K}$ (Kramida et al. 2020)... 32

Table 2.4: A comparison of the full-width half-maximum due to spectral broadening caused by the instrument, Doppler and collisional broadening that occurs during

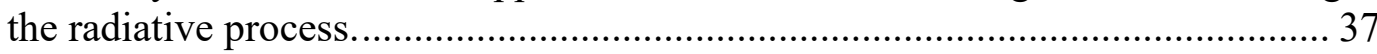

Table 3.1: The wavelength used to normalize relative spectral response curves. 49

Table 3.2: Summary of the lines that were used to assess wavelength shift for the narrowband and broad-band systems (Kaye 1997)................................................... 52

Table 3.3: A summary of the measured maximum extents of wavelength and wavenumber shifts for the narrow-band and broad-band systems using the $0.05 \mathrm{~mm}$ and $0.01 \mathrm{~mm}$ wide slits, respectively.

Table 3.4: Hydrocarbon fuel mixture typical of the Alberta UOG industry used during test to estimate the detectability of the $\mathrm{Na}$ and $\mathrm{K}$ first resonance doublet............ 56

Table 3.5: Flame and fuel / aerosol stream properties for the experiments used to estimate the detectability of $\mathrm{Na}$ and $\mathrm{K}$ as a function of salt loading rate.

Table 3.6: Summary of experiments to estimate the detection sensitivities of salt emission at a distance of $2 \mathrm{~m}$. The sensitivity limit of the broad-band system was estimated using the $0.01 \mathrm{~mm}$ wide slit. 60

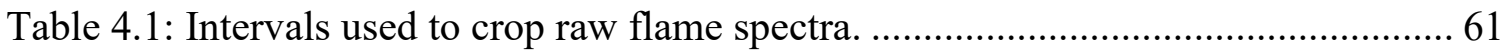

Table 4.2: Normalization wavelengths for calibrated spectra. 63

Table 4.3: Number of data points contained within the calibrated spectra and normalization wavenumbers for the resampled data in the wavenumber domain. 64

Table 4.4: Summary of the source and wavenumber range of spectra features that were removed from the wavenumber spectra to isolate soot emission.

Table 4.5: The wavenumber ranges that are used to calculate the standard deviation of measured intensity near the $\mathrm{Na}$ and $\mathrm{K}$ signatures.

Table 4.6: Window widths used to calculate the integrated area ratio for $\mathrm{Na}$ and $\mathrm{K}$ signatures. 
Table 4.7: Signal to noise ratio, integrate intensity ratio, and $\mathrm{P}$ value (from the $\mathrm{F}$ test) thresholds defining successful detections of $\mathrm{Na}$ and $\mathrm{K}$.

Table 5.1: The amount of flares found to have evidence of liquid carry-over in North Dakota, southern Saskatchewan, and Ecuador.

Table 5.2: Pearson correlation coefficient matrix for predictor variables: produced oil, water, and gas;, flared/vented gas; and mean well age, and minimum well age. 102

Table 5.3: The variance inflation factor (VIF) used to model the strength of collinearity among the six production parameters in a multivariable model that contains all six predictor variables (these include produced oil, water, and gas; flared/vented gas; and mean well age and minimum well age).....

Table 5.4: A summary of the correlations between species detections and the production parameters for well age, production volumes, and flared gas volumes. 107 


\section{List of Figures}

Figure 1.1: Separation vessels and storage tanks at a UOG facility in Ecuador. 19

Figure 1.2: Plot of concentrations of the most common components in found in produced water samples from the Williston basin as compiled in the database of (Blondes et al. 2018). The box represents the 25 and $75 \%$ percentiles of the data and the whiskers represent the 5 and $95 \%$ percentiles. 22

Figure 3.1: Image of the spectrometer-lens system. 42

Figure 3.2: The 3 main components of the Flame-S spectrometer: the interchangeable entrance slit, the optical bench, and the linear detector.

Figure 3.3: A diagram of the spectrometer measuring the monochromatic components of polychromatic light: (a) polychromatic light incident to diffraction grating, (b) monochromatic light reflected from DG onto linear detector.

Figure 3.4: Depiction of the FOV calculation for the narrow-band system.* Diagram not to scale.

Figure 3.5: Example of the type of type of streetlight that was used to fine focus the spectrometer system.

Figure 3.6: (a) A labelled diagram of the system used to calibrate the relative wavelength response of the spectrometers. (b) An example of the alignment between the spectrometer system and the integration sphere when performing a relative spectral response calibration. ${ }^{*}$ Not shown is the thick plastic sheet that was used to bridge the gap between the spectrometer system and the sphere to prevent stray light from entering the lens during a calibration.

Figure 3.7: Mean spectrometer readings of the calibrated integration sphere at approximately 25,50 , and $75 \%$ of the saturation limit of the spectrometer's linear detector for the (a) narrow-band spectrometer and (b) broad-band spectrometer with the $0.01 \mathrm{~mm}$ wide entrance slit (solid lines) and the $0.025 \mathrm{~mm}$ wide slit (dotted lines)

Figure 3.8: Relative spectral response curves (corresponding to approximately 25, 50, and $75 \%$ of the saturation limit of the spectrometer's linear detector). (a) Curves for the narrow-band spectrometer normalized by the intensity at $606 \mathrm{~nm}$. (b) Curves for the broad-band spectrometer with a $0.01 \mathrm{~mm}$ wide entrance slit normalized by the intensity at $900 \mathrm{~nm}$. Similar curves were obtained for the $0.025 \mathrm{~mm}$ slit but are not shown here.

Figure 3.9: One of the UVP PEN-RAY lamps that were used to assess the shift and skewness of measured spectral peaks, caused by the off-axis illumination of the spectrometer's optical bench 
Figure 3.10: The spectrometer system and Toughbook laptop setup at a measurement location where site access was granted.

Figure 3.11: The spectrometer system aimed at the lab-scale flare flame generated using the custom 2 in burner at the CUFF. The reflective igniter seen in the background was removed prior to performing the assessment. 58

Figure 3.12: Average sensitivity measurement spectrum that produced the sensitivity limit for the (a) narrow-band system and (b) broad-band systems when measuring $\mathrm{Na}$ and $\mathrm{K}$ emission, respectively. The sensitivity limit of the broad-band system was for the $0.01 \mathrm{~mm}$ wide slit. 60

Figure 4.1: Example measured flare spectra, average noise spectra, and noise-subtracted spectra for the (a) narrow-band system and (b) broad-band system (with a $0.01 \mathrm{~mm}$ slit).

Figure 4.2: The noise subtracted spectra from Figure 4.1, their unique calibration curves and the final calibrated spectra for the (a) narrow-band system and (b) broad-band system with a $0.01 \mathrm{~mm}$ slit. 63

Figure 4.3: The calibrated spectra from Figure $4.2(\mathrm{a} \& \mathrm{~b})$ and their re-sampled wavenumber spectrum for the (c) narrow-band system and (d) broad-band system with a $0.05 \mathrm{~mm}$ and $0.01 \mathrm{~mm}$ wide slit, respectively.

Figure 4.4: The wavenumber spectra from Figure 4.3 with excluded wavenumber intervals (grey) from Table 4.4 for (a) the narrow-band system with the $0.05 \mathrm{~mm}$ wide slit and (b) the broad-band system with the $0.01 \mathrm{~mm}$ wide slit.

Figure 4.5: Normalized and log-transformed spectra from Figure 4.4 and fitted OTN and OTK models for the (a) narrow-band system and (b) broad-band systems with a $0.05 \mathrm{~mm}$ and $0.01 \mathrm{~mm}$ wide slit, respectively.

Figure 4.6: The distributions of $T g$ using the narrow-band system (blue) and broad-band system (orange) for all flares measured in North Dakota / southern Saskatchewan (2019) and Ecuador (2020). Three flares measured with the narrow-band system were not measured with the broad-band system, and one narrow-band $T g$ value of $>2800 \mathrm{~K}$ was considered an outlier and was not included in the narrow-band histogram 70

Figure 4.7: Calculating the intensity of a resampled data point using LOESS in the removed data region of the $\mathrm{Na}$ first resonance doublet and the $\mathrm{K} / \mathrm{O}_{2}$ region for the (a) narrow-band spectrum and (b) broad-band spectrum from Figure 4.4, respectively. 72

Figure 4.8 (a) The measured narrow-band spectrum from Figure 4.7 with spectral features removed (orange), the interpolated background spectrum generated via the LOESS fit (blue), and a potential Na signature within the Na first resonance doublet region (black). (b) The isolated potential $\mathrm{Na}$ signature obtained by 
subtracting the blue curve from the black in curve (a) and theoretical locations of the spectral lines of the $\mathrm{Na}$ first resonance doublet (yellow).............................. 73

Figure 4.9: Fitted $\mathrm{Na}$ atomic emission model overlaid on the measured and isolated

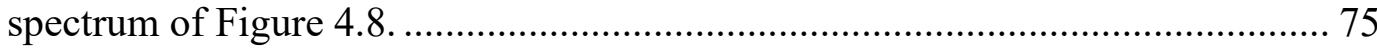

Figure 4.10: The measured broad-band spectrum, which includes $\mathrm{K}$ emission region (black) $\mathrm{O} 2$ absorption region (magenta), and background radiative emission of the flame (orange), and the LOESS interpolated background through the $\mathrm{K} / \mathrm{O}_{2}$ region (blue) 76

Figure 4.11: The molecular absorption model fitted to the $\mathrm{O}_{2} \mathrm{~A}$ band region in Figure 4.10 .

Figure 4.12: The atomic emission model fitted to the isolated $\mathrm{K}$ spectrum from Figure 4.11. Yellow lines indicated the theoretical locations of the $\mathrm{K}$ emission peaks. 78

Figure 4.13: The spectral regions from which standard deviation was calculated for a potential emission signature of (a) $\mathrm{Na}$ and (b) K.

Figure 4.14: (a) and (b) The region of measured spectrum that is included in the integrated intensity ratio analysis (the yellow curve) which had its background subtracted (the blue curve) for $\mathrm{Na}$ and $\mathrm{K}$, respectively. (c) and (d) The isolated spectrum of $\mathrm{Na}$ and $\mathrm{K}$ (wide), respectively. The fitted $\mathrm{O}_{2}$ absorption model (the magenta curve) is overlaid onto (d).

Figure 4.15: Example of the integrated intensity ratio calculation. The portions of the spectra from Figure 4.14 (c) and d that are contained within the peak window and a non-peak window are defined by the coloured lines. The shaded area under coloured lines represents the integrated intensity for those windows................. 83

Figure 4.16: Example spectra showing borderline successful (left panel) and null (right panel) detections of $\mathrm{Na}$ signatures in field data acquired during the 2019 field measurement campaign in North Dakota / Southern Saskatchewan and the 2020 field measurement campaign in Ecuador.

Figure 4.17: Example spectra showing borderline successful (left panel) and null (right panel) detections of $\mathrm{K}$ signatures in field data acquired during the 2019 field measurement campaign in North Dakota / Southern Saskatchewan and the 2020 field measurement campaign in Ecuador

Figure 5.1: The breakdown of detection status (by species) for flares measured in North Dakota / southern Saskatchewan in November 2019, Ecuador in February 2020, and their combined total.

Figure 5.2: The location and detection status of the flares measured in North Dakota and southern Saskatchewan in November 2019.

Figure 5.3: The location and detection status of the flares measured in Ecuador in February 2020. 
Figure 5.4: The effective flame temperature distributions for the flares with and without successful detections including a two tailed t-test comparing the mean flame temperature of these distributions. ${ }^{*}$ Excludes three flares that were not measured using the broad-band system.................................................................... 93

Figure 5.5: The (a) mean and (b) minimum well age of flares in North Dakota with successful and null species detections. In each case, the Mann-Whitney test is interpreted to compare population medians.

Figure 5.6: The total reported volume of (a) oil and (b) water produced by wells associated with flares with and without species detections, during the month of November, 2019. In each case, the Mann-Whitney test is interpreted to compare population medians. 98

Figure 5.7: The total reported volume of gas that was (a) produced and (b) flared/vented by wells associated with flares with and without species detections, during the month of November 2019. The Mann-Whitney test compares population medians for produced gas volumes, and the tendency for one population to produce larger values than the other for flared/vented gas volumes.

Figure 5.8: The true positive detection rate at $\alpha=0.05$ for six unique single-variable logistic regression models. A model was generated for each of the following production parameters: produced oil, water, and gas; flared/vented gas; and mean well age and minimum well age.

Figure 5.9: The true positive detection rate of models that were generated from every possible combination of the six production parameters. 106

Figure B.1: The age distributions of all active oil and gas well in North Dakota on November $8^{\text {th }}, 2019$ and the subset of wells that were linked to measured flares. 


\section{Nomenclature}

\begin{tabular}{|c|c|c|}
\hline Acronym & Description & $\begin{array}{l}\text { First } \\
\text { Use: } \\
\text { Page }\end{array}$ \\
\hline $\mathrm{AE}$ & Atomic emission & 67 \\
\hline ANSI & American National Standard Institute & 20 \\
\hline API & American Petroleum Institute & 20 \\
\hline CAC & Criteria air contaminants & 18 \\
\hline $\mathrm{Cl}$ & Chemiluminescence & 21 \\
\hline CUFF & Carleton University Flare Facility & 56 \\
\hline DSC & Days since completion & 95 \\
\hline EIA & Energy Information Administration (United States) & 18 \\
\hline EPA & Environmental Protection Agency (United States) & 23 \\
\hline FOV & Field of view & 44 \\
\hline GHG & Greenhouse gas & 18 \\
\hline GPS & Global Positioning System & 93 \\
\hline HITRAN & High-Resolution Transmission Molecular Absorption Database & 40 \\
\hline LOESS & Locally weighted regression & 71 \\
\hline MA & Molecular absorption & 67 \\
\hline NIR & Near-infrared spectrum & 67 \\
\hline NIST & National Institute of Standard and Technology (United States) & 31 \\
\hline OAQPS & Office of Air Quality Planning and Standards (United States) & 23 \\
\hline OTK & Optically thick & 26 \\
\hline OTN & Optically thin & 26 \\
\hline PDE & Partial differential equation & 28 \\
\hline PM & Particulate matter & 18 \\
\hline ROC & Receiver-operating characteristic & 104 \\
\hline RTE & Radiative transfer equation & 26 \\
\hline SSE & Residual sum of squared errors & 28 \\
\hline UOG & Upstream oil and gas & 18 \\
\hline UVP & Ultraviolet Products Company & 50 \\
\hline VIF & Variance inflation factor & 102 \\
\hline VIS & Visible spectrum & 102 \\
\hline VOC & Volatile organic compound & 18 \\
\hline $\begin{array}{l}\text { Chemical } \\
\text { Symbols }\end{array}$ & Description & $\begin{array}{l}\text { First } \\
\text { Use: } \\
\text { Page }\end{array}$ \\
\hline $\mathrm{Al}$ & Aluminum & 29 \\
\hline $\mathrm{BC}$ & Black carbon & 18 \\
\hline $\mathrm{C}_{2}$ & Carbon (gas) & 67 \\
\hline $\mathrm{C}_{2} \mathrm{H}_{6}$ & Propane & 56 \\
\hline $\mathrm{C}_{3} \mathrm{H}_{8}$ & Ethane & 56 \\
\hline
\end{tabular}




\begin{tabular}{|c|c|c|c|}
\hline $\begin{array}{l}\text { Chemica } \\
\text { Symbols }\end{array}$ & Description & & $\begin{array}{l}\text { First } \\
\text { Use: } \\
\text { Page }\end{array}$ \\
\hline $\mathrm{C}_{4} \mathrm{H}_{10}$ & Butane & & 56 \\
\hline $\mathrm{C}_{5} \mathrm{H}_{12}$ & Pentane & & 56 \\
\hline $\mathrm{C}_{6} \mathrm{H}_{14}$ & Hexane & & 56 \\
\hline $\mathrm{C}_{7} \mathrm{H}_{16}$ & Heptane & & 56 \\
\hline $\mathrm{Ca}$ & Calcium & & 29 \\
\hline $\mathrm{CH}_{4}$ & Methane & & 18 \\
\hline $\mathrm{Cl}$ & Chlorine & & 21 \\
\hline $\mathrm{CO}$ & Carbon monoxide & & 18 \\
\hline $\mathrm{CO}_{2}$ & Carbon dioxide & & 18 \\
\hline $\mathrm{H}_{2} \mathrm{O}$ & Water & & 29 \\
\hline K & Potassium & & 23 \\
\hline $\mathrm{KCl}$ & Potassium chloride & & 23 \\
\hline $\mathrm{Kr}$ & Krypton & & 50 \\
\hline $\mathrm{Mg}$ & Magnesium & & 29 \\
\hline $\mathrm{N}_{2}$ & Nitrogen (gas) & & 56 \\
\hline $\mathrm{Na}$ & Sodium & & 23 \\
\hline $\mathrm{NaCl}$ & Sodium chloride & & 22 \\
\hline $\mathrm{Ne}$ & Neon & & 50 \\
\hline $\mathrm{NO}_{x}$ & Nitrogen oxides (i.e., $\mathrm{NO} / \mathrm{NO}_{2}$ ) & & 18 \\
\hline $\mathrm{O}_{2}$ & Oxygen (gas) & & 24 \\
\hline $\mathrm{SO}_{\mathrm{x}}$ & Sulfur oxides (i.e., $\mathrm{SO} / \mathrm{SO}_{2}$ ) & & 18 \\
\hline $\begin{array}{c}\text { Latin } \\
\text { Symbol }\end{array}$ & Description & Units & $\begin{array}{l}\text { First } \\
\text { Use: } \\
\text { Page }\end{array}$ \\
\hline$A$ & $\begin{array}{l}\text { Normalized height of Gaussian probability density } \\
\text { function }\end{array}$ & {$[-]$} & 123 \\
\hline$A A E$ & Absorption Ångström Exponent & {$[-]$} & 27 \\
\hline$B$ & $\begin{array}{l}\text { Characterizes the spectral variability in the fraction } \\
\text { of soot extinction due to absorption, also denotes } \\
\text { the broad-band spectrometer in Table } 4.4\end{array}$ & {$[\mathrm{~cm}]$} & 27 \\
\hline$c$ & Speed of light in a vacuum, $c=2.998 \times 10^{8} \mathrm{~m} \mathrm{~s}^{-1}$ & {$\left[\mathrm{~m} \mathrm{~s}^{-1}\right]$} & 26 \\
\hline$C$ & $\begin{array}{l}\text { Optical conductance of spectrometer system, also } \\
\text { used to scale atomic emission model }\end{array}$ & {$\left[\mathrm{m}^{-2} \mathrm{sr}^{-1}\right]$} & 34 \\
\hline$d$ & Stand-off distance & {$[\mathrm{m}]$} & 44 \\
\hline$d \tilde{v}$ & Wavenumber shift term & {$\left[\mathrm{cm}^{-1}\right]$} & 73 \\
\hline$D$ & Term used to scale molecular absorption model & {$[-]$} & 39 \\
\hline$D F$ & Degrees of freedom for $F$ test & {$[-]$} & 84 \\
\hline$e$ & Ionized electron & {$[-]$} & 30 \\
\hline$E^{\prime}, E^{\prime \prime}$ & $\begin{array}{l}\text { Energy of lower and upper states involved with } \\
\text { quantum state transition }\end{array}$ & {$[\mathrm{J}]$} & 31 \\
\hline$f$ & Focal length of plano-convex lens & {$[\mathrm{mm}]$} & 44 \\
\hline$h$ & Planck's constant, $h=6.626 \times 10^{-34} \mathrm{~J} \mathrm{~s}$ & {$[\mathrm{~J} \mathrm{~s}]$} & 26 \\
\hline
\end{tabular}




\begin{tabular}{|c|c|c|c|}
\hline $\begin{array}{l}\text { Latin } \\
\text { Symbol }\end{array}$ & Description & Units & $\begin{array}{l}\text { First } \\
\text { Use: } \\
\text { Page }\end{array}$ \\
\hline$H$ & Height of spectrometer slit and system FOV & {$[\mathrm{mm}, \mathrm{m}]$} & 44 \\
\hline$H_{o}, H_{a}$ & $\begin{array}{l}\text { Null and alternative hypotheses for statistical } \\
\text { hypothesis test }\end{array}$ & {$[-]$} & 84 \\
\hline$I$ & Spectral radiance at spectrometer location & {$\left[\mathrm{W} \mathrm{m} \mathrm{m}^{-2} \mathrm{sr}^{-1}\left(\mathrm{~cm}^{-1}\right)^{-1}\right]$} & 27 \\
\hline$I_{B B-P}$ & Planck's blackbody equation & {$\left[\mathrm{W} \mathrm{m} \mathrm{m}^{-2} \mathrm{sr}^{-1}\left(\mathrm{~cm}^{-1}\right)^{-1}\right]$} & 33 \\
\hline$I_{B B-W}$ & Wien's approximation & {$\left[\mathrm{W} \mathrm{m} \mathrm{m}^{-2} \mathrm{sr}^{-1}\left(\mathrm{~cm}^{-1}\right)^{-1}\right]$} & 26 \\
\hline$I_{\text {interp }}$ & Interpolated background & [a.u.] & 39 \\
\hline$I_{o}$ & Incident intensity for molecular absorption model & {$\left[\mathrm{W} \mathrm{m} \mathrm{m}^{-2} \mathrm{sr}^{-1}\left(\mathrm{~cm}^{-1}\right)^{-1}\right]$} & 38 \\
\hline$I_{t}$ & $\begin{array}{l}\text { Transmitted intensity for molecular absorption } \\
\text { model }\end{array}$ & {$\left[\mathrm{W} \mathrm{m}{ }^{-2} \mathrm{sr}^{-1}\left(\mathrm{~cm}^{-1}\right)^{-1}\right]$} & 38 \\
\hline$I_{\text {Raw }}$ & Raw spectrometer readings & [counts pixel $^{-1}$ ] & 34 \\
\hline$I_{\text {Rel }}$ & Shape-corrected spectrometer readings & [a.u.] & 35 \\
\hline$k$ & Spectral absorption coefficient & [(atom or mol.) $\left.\mathrm{cm}^{-1}\right]$ & 35 \\
\hline$k_{B}$ & Boltzmann's constant $k_{B}=1.3806 \times 10^{-23} \mathrm{~J} \mathrm{~K}^{-1}$ & {$\left[\mathrm{~J} \mathrm{~K}^{-1}\right]$} & 26 \\
\hline$L$ & Path-length through flame (along line-of-site) & {$[\mathrm{m}]$} & 26 \\
\hline$M$ & Vertical shift term for least squares regression & [a.u.] & 73 \\
\hline$n$ & Number density of gas & [(atom or mol. $\left.) \mathrm{cm}^{-3}\right]$ & 74 \\
\hline $\bar{n}$ & Path-averaged concentration of soot & {$[-]$} & 27 \\
\hline$N$ & $\begin{array}{l}\text { Number of parameters for } \mathrm{F} \text { test, also denotes the } \\
\text { narrow-band spectrometer in Table } 4.4\end{array}$ & {$[-]$} & 67 \\
\hline 0 & Least squares objective function & {$[-]$} & 28 \\
\hline$P$ & Number of fitted parameters for $F$ test & {$[-]$} & 84 \\
\hline$s$ & Properties related to spectrometer's entrance slit & {$[\mathrm{m}]$} & 44 \\
\hline$S$ & Linestrength function & $\left.\left[\mathrm{cm}^{3} \text { (atom or mol. }\right)^{-1}\right]$ & 74 \\
\hline$S S E$ & Residual sum of squared error for regression & {$[-]$} & 28 \\
\hline$T$ & Soot blackbody temperature & {$[\mathrm{K}]$} & 26 \\
\hline$T_{a m b}$ & Ambient temperature of measurement & {$[\mathrm{K}]$} & 77 \\
\hline$T_{g}$ & $\begin{array}{l}\text { Instantaneous local gas kinetic temperature of } \\
\text { flame }\end{array}$ & {$[\mathrm{K}]$} & 26 \\
\hline$T_{\text {elec }}$ & Electronic temperature & {$[\mathrm{K}]$} & 33 \\
\hline$T_{e q l}$ & Equilibrium temperature & {$[\mathrm{K}]$} & 32 \\
\hline $\begin{array}{l}T_{\text {OTN }} \\
T_{\text {OTK }}\end{array}$ & Soot temperature for optically thin and thick flame & {$[\mathrm{K}]$} & 28 \\
\hline$\tilde{v}$ & Wavenumber & {$\left[\mathrm{cm}^{-1}\right]$} & 26 \\
\hline$\tilde{v}_{O}$ & Reference wavenumber & {$\left[\mathrm{cm}^{-1}\right]$} & 27 \\
\hline$x$ & Volume fraction of thermal radiator & {$[-]$} & 36 \\
\hline$X$ & Normalized soot spectrum wavenumber & {$[-]$} & 28 \\
\hline$Y$ & Normalized soot spectrum intensity & {$[-]$} & 28 \\
\hline$W$ & Width of spectrometer slit and system FOV & {$[\mathrm{mm}, \mathrm{m}]$} & 44 \\
\hline
\end{tabular}




\begin{tabular}{|c|c|c|c|}
\hline $\begin{array}{l}\text { Greek } \\
\text { Symbol }\end{array}$ & Description & Units & $\begin{array}{l}\text { First } \\
\text { Use: } \\
\text { Page }\end{array}$ \\
\hline$\alpha$ & $\begin{array}{l}\text { Type } 1 \text { error rate for statistical hypothesis tests, also } \\
\text { absorptivity in Eq. (2.17) }\end{array}$ & {$[-]$} & 35 \\
\hline$\beta$ & Fitted parameter for logistic regression model & {$[-]$} & 101 \\
\hline$\gamma_{I P}$ & Full-width half-max for instrument profile kernel & {$[-]$} & 34 \\
\hline$\gamma_{C}$ & Full-width half-max for collisional broadening & {$\left[\mathrm{cm}^{-1}\right]$} & 37 \\
\hline$\gamma_{D}$ & Full-width half-max for Doppler broadening & {$\left[\mathrm{cm}^{-1}\right]$} & 37 \\
\hline$\varepsilon$ & Emissivity & {$[-]$} & 33 \\
\hline$\zeta$ & $\begin{array}{l}\text { Placeholder for exponential term in soot temperature } \\
\text { equation (Eq. (2.2)) }\end{array}$ & {$[-]$} & 27 \\
\hline$\eta$ & $\begin{array}{l}\text { Placeholder for multiplicative term in soot temperature } \\
\text { equation (Eq. (2.2)) }\end{array}$ & {$[-]$} & 27 \\
\hline$\kappa_{a}$ & Spectral absorption coefficient & {$\left[\mathrm{m}^{-1}\right]$} & 119 \\
\hline$\kappa_{e}$ & Spectral extinction coefficient & {$\left[\mathrm{m}^{-1}\right]$} & 119 \\
\hline$\sigma_{a}$ & Spectral absorption cross section of soot & {$\left[\mathrm{m}^{2}\right]$} & 119 \\
\hline$\sigma_{e}$ & Spectral extinction cross section of soot & {$\left[\mathrm{m}^{2}\right]$} & 119 \\
\hline$\tau$ & Transmissivity & {$[-]$} & 35 \\
\hline$\Phi_{I P}$ & $\begin{array}{l}\text { Gaussian kernel for spectral broadening due to instrument } \\
\text { profile }\end{array}$ & {$[-]$} & 34 \\
\hline$\phi$ & Lineshape function & {$[\mathrm{cm}]$} & 37 \\
\hline$\Psi$ & Relative spectral response curve & {$[-]$} & 34 \\
\hline$\widetilde{\omega}$ & $\begin{array}{l}\text { Ratio of spectral absorption-to-extinction cross sections of } \\
\text { soot }\end{array}$ & {$[-]$} & 120 \\
\hline
\end{tabular}




\section{Chapter 1 Introduction}

\subsection{Gas Flaring and Entrainment of Aerosols into Flare Systems}

\subsubsection{Flaring in the Upstream Oil and Gas Sector}

In the upstream oil and gas (UOG) industry, associated gas that is co-produced during oil production may be captured for further processing or flared. Flaring is used to dispose of associated gas in oil producing regions where gas capture or re-injection are not feasible due to technical, economic, and/or regulatory challenges (World Bank 2020a). A flare system relies on open atmosphere combustion to destroy methane $\left(\mathrm{CH}_{4}\right.$, a potent greenhouse gas (GHG)) and other volatile organic compounds (VOCs) in the associated gas stream, but leads to the emission of other combustion by-products. These by-products of hydrocarbon combustion may include carbon dioxide $\left(\mathrm{CO}_{2}\right)$ and various criteria air contaminants $(\mathrm{CAC})$ such as nitrogen oxides $\left(\mathrm{NO}_{\mathrm{x}}\right)$, carbon monoxide $(\mathrm{CO})$, sulfur oxides $\left(\mathrm{SO}_{\mathrm{x}}\right)$, and particulate matter (PM, i.e., black carbon (BC) or soot) (Conrad and Johnson 2017; Knighton et al. 2012; Torres et al. 2012; Johnson et al. 2001; Johnson and Kostiuk 2000; Strosher 2000). The emission of $\mathrm{CH}_{4}$, various VOCs, and some CACs may be amplified by the incomplete combustion of associated gas, which is sensitive to a variable fuel stream velocity and/or turbulent crosswinds (Burtt et al. 2020; Johnson and Kostiuk 2002).

It is important to limit the emissions of UOG flares because $\mathrm{CH}_{4}$ and $\mathrm{CO}_{2}$ are influential greenhouse gases, the various CACs pose a risk to human health and the environment, and $\mathrm{BC}$ has both strong climate forcing effects and adverse heath impacts (Stohl et al. 2013; U.S. EPA 2021; Li et al. 2016). The World Bank estimated that in 2019 approximately 150 billion cubic meters of associated gas was flared globally, of which the U.S.A. was responsible for $\sim 12 \%$ - making it the third largest contributing country that year (World Bank 2020b). The U.S. Energy Information administration (EIA) reported that in 2019 the state of North Dakota was responsible for $\sim 38 \%$ all vented and flared natural gas in the USA, second to only Texas (U.S. EIA 2019). Furthermore, Cushing et al. (2021) have found that the North Dakota region of the Willison basin has the highest density of flares 
in the U.S.A. with a maximum flare density of 716 [flares $\left.\left(\mathrm{km}^{2}\right)^{-1}\right]$. Cushing et al. (2021) go on to estimate that approximately half a million people in the U.S.A live within $5 \mathrm{~km}$ of UOG industry flaring leaving them vulnerable to numerous flare emissions that have adverse health effects.

\subsubsection{Separation of Produced Liquids from Flare Gas at Upstream Oil and Gas Production Facility}

A typical well at a UOG facility will co-produce a mixture of fluids which may include crude oil, natural gas, flowback fluids (if the well has been hydraulically fractured), and formation water. These components make up the production stream which is typically separated upon reaching the surface. The separation process may be implemented in different ways, but it is common to use separation vessels to segregate the produced fluids into oil, water, and gas streams. The oil and water may then be directed into pipelines or on-site storage tanks prior to further processing or transport. The gas stream may similarly be captured for on site use or into pipelines for transport, but is often directed to an on site flare for disposal. Figure 1.1 shows a photograph of separation vessels at a multi-well UOG facility in Ecuador.

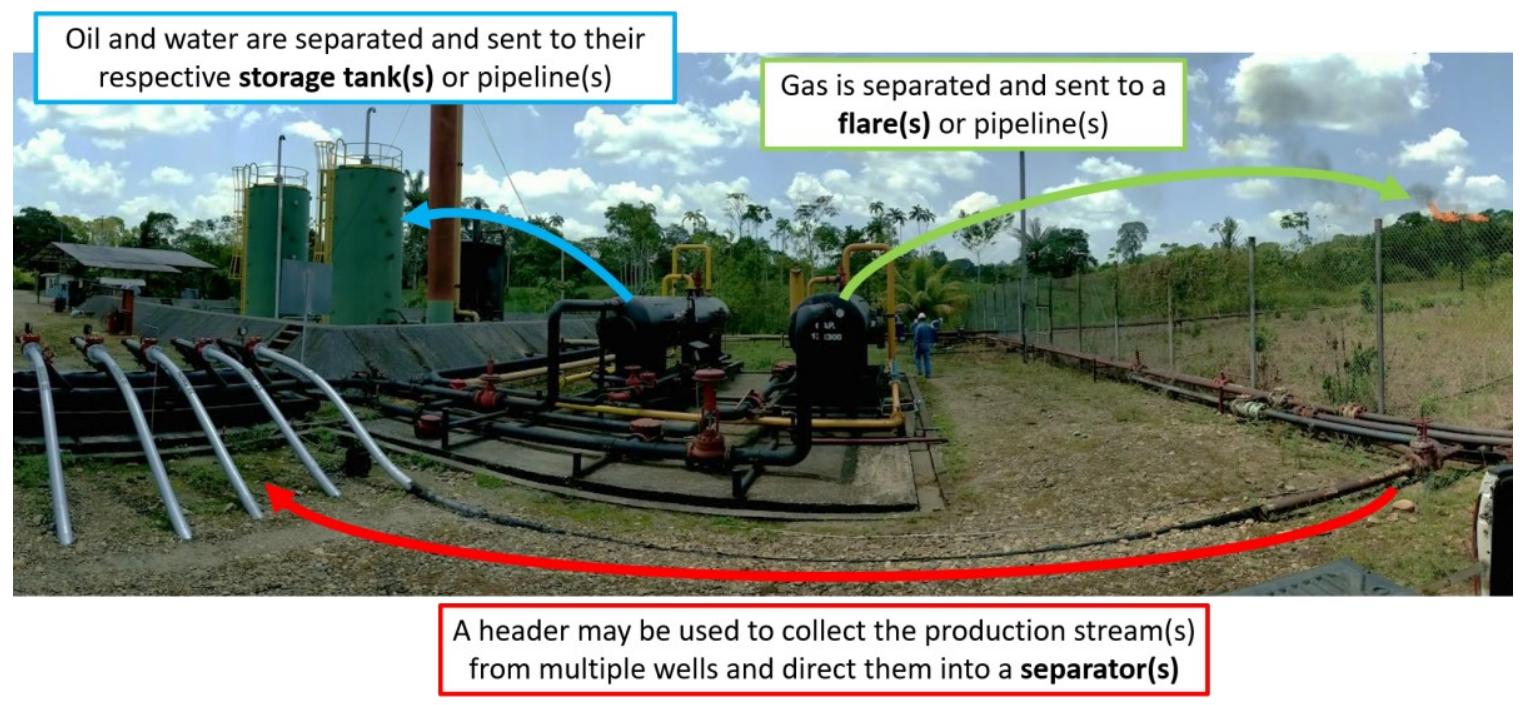

Figure 1.1: Separation vessels and storage tanks at a UOG facility in Ecuador.

Unfortunately, the separation process is not perfect and anecdotal information suggests it may be common for liquids to pass through the separation vessel and be entrained as aerosols in the gas stream. Shaban (1995) suggested that the separation process at UOG 
facilities may become strained when foam is present in the separator. Specifically, the formation of foam and the operational adjustments required to control it have been observed to reduce separator efficiency, which may lead to the "carry-over" of crude oil into the gas stream (Shaban 1995).

Anecdotally, there are few literature resources that address the problem of liquid carryover at UOG facilities and, to the author's knowledge, none that have studied the degree and/or frequency in which it occurs in practice. The problem of liquid carry-over at UOG facilities is partially addressed by ANSI/API Standard 521, which provides guidance for designing on-site separation equipment and flare systems. ANSI/API Standard 521 emphasises the importance of achieving maximum liquid-gas disengagement because flares are typically not designed to efficiently combust droplets of liquid hydrocarbon (API 2014). This standard recommends that on-site separation tanks be sized to remove droplets 300 to $600 \mu \mathrm{m}$ in diameter from the gas stream, warning of such consequences as excessive smoking due to incomplete combustion, possible "burning rain", and flame out (API 2014). The ANSI/API Standard 521 influences jurisdictional bodies that regulate oil and gas operations in U.S.A., Canada, Columbia, Mexico, Indonesia, Singapore, Nigeria, and Malaysia (API 2020).

\subsubsection{The Formation Processes of Liquid Droplets in the Production Stream at Upstream Oil and Gas Facilities}

There are at least 3 important mechanisms by which liquid aerosols may become suspended in the associated gas prior to and during the separation process: condensation, atomization, and reentrainment (e.g., a liquid slug shearing from the gas-liquid interface of a co-flowing stream and being transported within the gas phase) (Wines and Brown 1994). The approximate droplet diameters produced by each mechanism are summarized in Table 1.1, which suggests that all mechanisms are capable of producing aerosols small enough to bypass a separation tank designed to remove droplets greater than $600 \mu \mathrm{m}$ in diameter.

To pass through the separator, the aerosols suspended in the associated gas will have to escape one or more of the separation methods that are commonly used by separation tanks: momentum separation, gravity settling, or coalescing (Shaban 1995). Ignoring the 
presence of foam in the separator, the effectiveness of these separation methods relies on the separator's temperature and pressure, the gas and aerosol composition, and the gas velocity and turbulence (Shaban 1995). If the conditions that promote aerosol suspension are present (i.e., a high gas density, a high gas velocity, and a turbulent gas stream), it is then reasonable to assume that some amount of produced oil and produced water aerosols could be carried through the separator and into the flare system and ultimately its flame. However, the prevalence of this occurrence at upstream flares in the field is currently unknown, and indeed is the motivation for the current work.

Table 1.1: Important mechanisms for aerosl formation and their estimated droplet diameters (Wines and Brown 1994).

\begin{tabular}{|l|c|}
\hline Aerosol Formation Mechanism & Approximate Droplet Diameter $[\boldsymbol{\mu m}]$ \\
\hline Condensation & $0.2-5$ \\
\hline Atomization & $10-200$ \\
\hline Reentrainment & $500-5000$ \\
\hline
\end{tabular}

\subsection{Potential Effects of Produced Water Aerosols in Flare Flames}

Produced water at UOG facilities is typically "brackish" meaning it has a high concentration of dissolved solids (i.e., salts) (Stanton et al. 2017). Liquid aerosols entrained into flares systems are expected to match the composition of produced water. The concentrations of the strongest components of produced water produced by UOG facilities in the Williston basin of North Dakota are shown in Figure 1.2. Noting the logarithmic scale on the vertical axis, the sodium $\left(\mathrm{Na}^{+}\right)$and chlorine $\left(\mathrm{Cl}^{-}\right)$ions are the dominant dissolved components, with concentrations $\sim 10 \times$ greater than the next most abundant component, the calcium ion $\left(\mathrm{Ca}^{+2}\right)$. The relative strength of $\mathrm{Na}^{+}$and $\mathrm{Cl}^{-}$in produced water suggests that if produced water aerosols were carried into a flare system, these species are the most likely to affect flare emissions. 


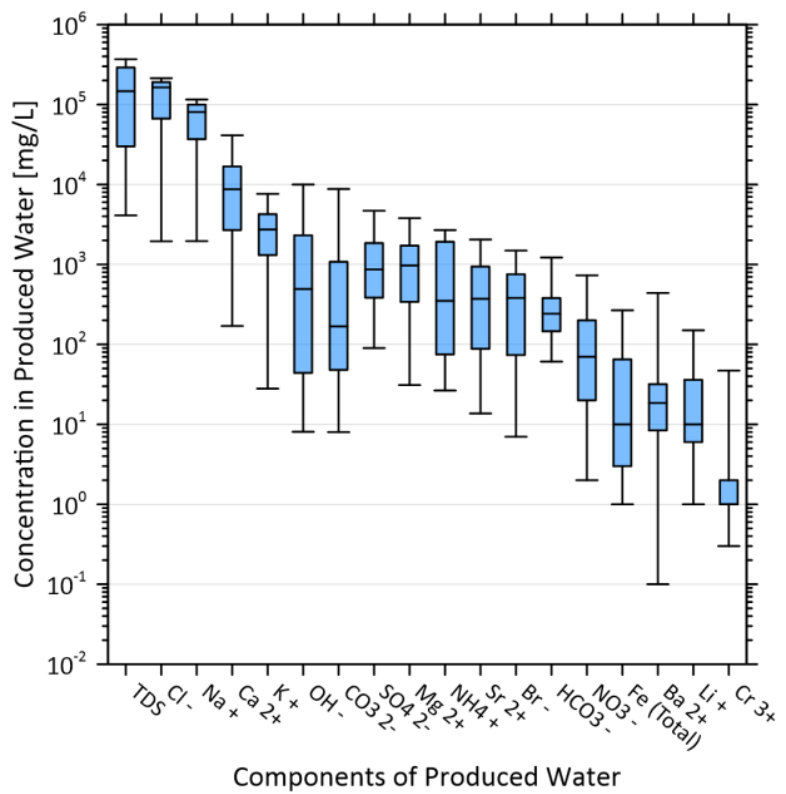

Figure 1.2: Plot of concentrations of the most common components in found in produced water samples from the Williston basin as compiled in the database of (Blondes et al. 2018). The box represents the 25 and $75 \%$ percentiles of the data and the whiskers represent the 5 and $95 \%$ percentiles.

Several recent and ongoing studies have considered the potential effects of entrained salt species on flare emissions by introducing sodium chloride $(\mathrm{NaCl})$ into hydrocarbon-fueled lab-scale flames. In general, these studies have found that $\mathrm{NaCl}$ has the potential to significantly augment CO, benzene, and particulate emissions (Jefferson 2017; Kazemimanesh et al. 2014 \& 2020; Roth et al. 2020; Trivanovic et al. 2019). However, these lab-scale experiments are only suggestive since the frequency and severity with which $\mathrm{NaCl}$ laden produced water aerosols are carried into flare systems at UOG facilities are unknown. Nevertheless, given that produced water is typically a majority component in UOG production streams in regions like North Dakota, separators that are not running optimally may have the potential to sustain liquid carry-over (Veil 2020).

There are also potential adverse impacts of entraining liquid hydrocarbon aerosols into flare systems. Hydrocarbon liquid carry-over was indirectly studied by Strosher (2000) who focused on the effect that co-flowing natural gas and industrial condensate streams had on the combustion efficiency of flares at a UOG facility in Canada. The study found that various concentrations of liquid fuel in a co-flowing fuel stream could lead to a reduction in flare flame combustion efficiency to, at one site, 64 to $71 \%$. It is therefore possible that a co-flowing fuel stream could reduce the combustion efficiency of a flare 
below 96.5\%, which according to U.S. EPA, constitutes good flare performance of a properly operated flare (U.S. EPA and OAQPS 2012).

\subsection{Proposed Method of Confirming the Presence of Non-Hydrocarbon Liquid in Flare Flames via Atomic Emission Spectroscopy}

To the author's knowledge, there are no established techniques for identifying nonhydrocarbon liquids in flare flames at upstream production facilities. This means there are no available data on whether non-hydrocarbon aerosols can be carried into flare systems, how often this may occur (if it does occur), or the degree with which it occurs. Given the potential adverse pollution impacts of entraining liquid aerosols, especially salt-laden aerosols, into flare systems as observed in the lab-based studies cited above, there is an urgent need to understand first whether aerosol entrainment is common under field conditions. This is especially true considering that droplet size in these studies was significantly smaller than the minimum 300 to $600 \mu \mathrm{m}$ diameter separation criteria in API 521.

Flame emission spectroscopy theory suggests that if aerosols of $\mathrm{NaCl}$ and potassium chloride $(\mathrm{KCl})$ solutions are present in an UOG flare flame, they may be identifiable via the atomic emission of Na and K (Alkemade et al. 1970; Gibson et al. 1963; Gaydon 1974). The atomic emission of $\mathrm{Na}$ and $\mathrm{K}$ are known to produce persistent spectral doublets at 588.99 / 589.59 and $766.48 / 769.89 \mathrm{~nm}$, respectively (Kramida et al. 2020). It is possible then that the radiative emission from a UOG flare flame could be measured using a spectrometer and the resulting spectra analysed for suspected emission signatures at the wavelengths of persistent $\mathrm{Na}$ and $\mathrm{K}$ emission. A suspected emission signature with a profile and location that matches the spectral doublet of $\mathrm{Na}$ or $\mathrm{K}$ may then prove the presence of a target species in the flame and provide strong evidence of liquid carry-over in real-world operating flare systems.

\subsection{Thesis Objectives and Outline}

\subsubsection{Objectives}

The objectives of this thesis were to develop and deploy a spectrometer system capable of detecting the atomic emission of gaseous $\mathrm{Na}$ and $\mathrm{K}$ flare flames from the fence-line of 
UOG facilities. The atomic emission signatures of $\mathrm{Na}$ and $\mathrm{K}$ were chosen as potential indicators of liquid carry-over because they are typically present in produced water in relatively strong concentrations, their salt compounds $(\mathrm{NaCl}$ and $\mathrm{KCl})$ have boiling temperatures (See Table 2.2) below the flame temperature of hydrocarbon-fueled flame $(<2300 \mathrm{~K})$, and each species emits a very persistent spectral doublet in the visible spectrum.

The second main objective of this thesis was to perform field experiments to determine the frequency in which liquid carry-over occurs at operational UOG facilities. Using these

data, a third main objective was to attempt to assess whether the likelihood of liquid carryover was correlated with, or could be classified by well age or production characteristics.

\subsubsection{Thesis Outline}

Chapter 2 introduces the theory behind the radiative emission of flare flames which includes the continuous emission of incandescing soot, the atomic emission of $\mathrm{Na}$ and $\mathrm{K}$, and the molecular absorption of oxygen $\left(\mathrm{O}_{2}\right)$. Chapter 3 details the developed spectrometer system and summarizes the relative spectral response calibration, the wavelength shift assessment, tests to preliminarily set a bound on the minimum detection limit, and flare field measurement protocols. Chapter 4 details the procedure used to process flare spectra while providing a sample spectrum for each step. Chapter 5 presents the results from the investigation into the frequency of liquid carry-over in UOG flare flames in North Dakota, southern Saskatchewan, and Ecuador. The results from the exploratory assessment of potential indicators of liquid carry-over in North Dakota are also included. Finally, Chapter 6 concludes the thesis and provided recommendations for future work. 


\section{Chapter 2 Review of Atomic Emission Spectroscopy as Applicable to a Remote Flare Measurement}

The combustion zone within upstream oil and gas (UOG) flares is a chemically complex environment. A series of exothermic chemical reactions bring the flare gas through pyrolysis and oxidation, increasing the translational kinetic energy of gas species, which is the reason for high combustion temperatures. Under these conditions, the atoms and molecules that make up the reactants, intermediate species, and products in these reactions may be briefly raised from their ground energy state to a higher energy state. Species transition between upper and low energy states by three main processes: chemical reactions, collisions, and the absorption or emission of a photon (electromagnetic packet of energy) (Alkemade et al. 1982). This chapter reviews the fundamental theory of atomic and molecular spectroscopy and solid particle incandescence as relevant to the detection of non-hydrocarbon species in a hydrocarbon-fueled flame.

\subsection{Blackbody Emission}

Black carbon $(\mathrm{BC})$ or soot is a form of particulate matter $(\mathrm{PM})$ that results from incomplete combustion of hydrocarbon fuels (e.g., Lou et al. 2010). The atoms that compose solid soot particles may absorb energy via collisions with flame gas species. If the resulting increase in kinetic energy of the soot atoms shifts to their electrons, that atom may occupy an excited electronic state. Unlike combustion-relevant gaseous species, the energy level of the excited state is not quantized, due to the physicochemical structure of soot particulate. Therefore, when soot atoms/electrons are de-excited through radiative emission, the emitted radiation is spectrally continuous (MacIsaac et al. 1999).

The spectral radiance $\left[\mathrm{W} \mathrm{m}{ }^{-2} \mathrm{sr}^{-1}\left(\mathrm{~cm}^{-1}\right)^{-1}\right]$ of soot's continuous emission spectrum is considered to follow Planck's law (Mekhrengin et al. 2019; Snelling et al. 2005). For flame temperatures typical of UOG flares (i.e., $<2300 \mathrm{~K}$ ) and for wavenumbers that are relevant to this study 11111 to $25000 \mathrm{~cm}^{-1}$ (400 to $900 \mathrm{~nm}$ ), Planck's blackbody equation may be simplified via Wien's approximation to within 1\% accuracy (Alkemade et al. 1982). Using 
Wien's approximation, the spectral radiance of blackbody emission $\left(I_{B B-W}\right)$ is (Alkemade et al. 1982):

$$
I_{B B-W}(\tilde{v}, T)=\left(2 \times 10^{8}\right) h c^{2} \tilde{v}^{3} e^{-\frac{h c \tilde{v}}{k_{B} T} \times 100}
$$

where $h$ is Planck's constant $\left[6.626 \times 10^{-34} \mathrm{~J} \mathrm{~s}\right], c$ is the speed of light in a vacuum $\left[2.998 \times 10^{8} \mathrm{~m} \mathrm{~s}^{-1}\right], k_{B}$ is Boltzmann's constant $\left[1.3806 \times 10^{-23} \mathrm{~J} \mathrm{~K}^{-1}\right], \tilde{v}$ is the wavenumber $\left[\mathrm{cm}^{-1}\right]$, and $T$ is the blackbody temperature $[\mathrm{K}]$ (i.e., the temperature of the emitting soot particles) (Alkemade et al. 1982). Mekhrengin et al. (2019) showed that soot temperatures deduced from optical techniques utilizing Wien' approximation closely match the temperature measured by a thermocouple (within $1.2 \%$ ). Since thermometric instruments are often used to measure gas kinetic temperature, it is assumed that $T$ is an adequate measure of the instantaneous local gas kinetic temperature of the flame, $T_{g}$ (Chandra Sekharan 2018). The soot temperature is assumed to adhere to the Maxwellian distribution, which describes the distribution of translational energy among the population of soot particles (Macheret et al. 2002). The soot temperature may ultimately be used to model atomic emission by assuming a state of local thermodynamic equilibrium in the flare flame, which is discussed in section 2.3.

\subsubsection{Calculating Instantaneous Gas Kinetic Temperature from Soot Spectra using Radiative Transfer Theory}

This section describes how $T_{g}$ was estimated from flare spectra using a modified version of the radiative transfer equation (RTE) for soot emission, which was derived by Dr. Bradley Conrad, a post doctoral researcher in the Energy \& Emissions Research Lab at Carleton University (see Appendix A, Section A.1 for the details of this derivation). Under field conditions, the position of the flame is highly variable and the thickness and soot volume fraction along the optical axis is unknown. Two bounding cases were considered that modelled the flame as either optically thin (OTN) or optically thick (OTK). The optical thickness of a flare flame refers to the path length through flame $(L[\mathrm{~m}])$ and fundamentally describes its ability to transmit light without absorption (Alkemade et al. 1982). The OTN case assumes $L \rightarrow 0$ whereas the OTK case assumes $L \rightarrow \infty$. The general form of soot 
radiative emission from an optically thick and optically thin flame is equal to Eq. (A.17) in Appendix A, Section A.1:

$$
\frac{I(\tilde{v})}{I\left(\tilde{v}_{o}\right)}=\left(\frac{\tilde{v}}{\tilde{v}_{o}}\right)^{\zeta} e^{\left(\eta\left(1-\frac{\widetilde{v}}{\tilde{v}_{o}}\right)\right)}
$$

where $I$ is the incident spectral intensity of soot emission at the spectrometer location $\left[\mathrm{W} \mathrm{m}{ }^{-2} \mathrm{sr}^{-1}\left(\mathrm{~cm}^{-1}\right)^{-1}\right], \tilde{v}_{o}$ is some reference wavenumber $\left[\mathrm{cm}^{-1}\right]$, and the general variables $\zeta, \eta$, and $T$ are summarized in Table 2.1 for an OTN and OTK flame.

Table 2.1: The values of the general variables found in Eq. (2.2).

\begin{tabular}{|l|c|c|}
\hline Model & $\zeta$ & $\boldsymbol{\eta}$ \\
\hline OTN & $3+A A E$ & $\frac{h c \tilde{v}_{O}}{k_{B} T_{\text {OTN }}}$ \\
\hline OTK & 3 & $\frac{h c \tilde{v}_{O}}{k_{B} T_{\text {OTK }}}-B \tilde{v}_{O}$ \\
\hline
\end{tabular}

In Table 2.1, AAE [-] is the "Absorption Ångström Exponent" which characterizes the spectral variability of absorption via a power-law; and $B[\mathrm{~cm}]$ is a constant characterizing the spectral variability in the fraction of soot extinction due to absorption (Conrad (2021) by way of Johnson et al. (2013) and Schnaiter et al. 2003). Reasonable values for $A A E$ and $B$ are presented in the form of normal distributions in Appendix A, Section A.1 (Conrad 2021 by way of van de Hulst (1957) and Johnson et al. (2013)).

The OTN and OTK models were derived from an RTE model that did not consider ambient light meaning that, in this case, optical thickness affects only the spectral intensity of soot emission from the flame. Furthermore, because the OTN and OTK models are normalized by some reference wavenumber, the effect of optical thickness is measured via the relative spectral shape of soot emission. The advantage of these OTN and OTK models is that, at these limits, unknown variables from the RTE (i.e., $L$ and the path-averaged concentration of soot, $\bar{n}[-])$ drop out so that soot temperature could be optimized via least squares regression.

The temperature of a soot spectrum was found via least squares regression of the OTN and OTK models of Eq. (2.2) using the variable values shown in Table 2.1. The least squares 
regression was performed by first taking the log-transform of the OTN and OTK models and the soot spectrum to convert their inherent exponential trend into a linear trend. The log-transform of Eq. (2.2) is:

$$
\ln Y=\zeta \ln X+\eta(1-X)
$$

where:

$$
\begin{gathered}
Y=\frac{I(\tilde{v})}{I\left(\tilde{v}_{o}\right)} \\
X=\frac{\tilde{v}}{\tilde{v}_{o}}
\end{gathered}
$$

The linear form of the OTN and OTK models (i.e., Eq. (2.3)) was then be substituted into the least squares objective function describing the sum of squared errors between observed and predicted values (Dekking et al. 2005):

$$
O=\sum(\zeta \ln X+\eta(1-X)-\ln Y)^{2}
$$

The value of $\eta$ that minimizes Eq. (2.6) was found by first deriving a partial differential equation (PDE) with respect to $\eta$ (Dekking et al. 2005):

$$
\eta=\frac{\sum \ln Y \ln (X)-\zeta \sum \ln (X)^{2}}{\sum \ln (X)(1-X)}
$$

Using the OTN or OTK expressions in Table 2.1, a value of $\zeta$ was substituted into Eq. (2.7) to solve for $\eta$. Then, values of $T_{\text {OTN }}$ or $T_{\text {OTK }}$ were determined by setting $\eta$ equal to the OTN or OTK expression for $\eta$ in Table 2.1 and solving.

To determine the representative $T_{g}$, a weighted average of the range of soot spectrum temperatures ( $T_{\text {OTN }}$ and $T_{\text {OTK }}$ ) was used as shown in Eq. (2.8). This weighted averaged considered the sum of squared errors (SSE) of the fitted OTN and OTK models: 


$$
T_{g}=\frac{\left(T_{\text {OTN }}\left(\frac{1}{S S E_{\text {OTN }}}\right)\right)+\left(T_{\text {OTK }}\left(\frac{1}{S S E_{\text {OTK }}}\right)\right)}{\left(\frac{1}{S S E_{\text {OTN }}}\right)+\left(\frac{1}{S S E_{\text {OTK }}}\right)}
$$

As discussed in Chapter 4, the coefficient of determination $\left(\mathrm{r}^{2}\right.$ value $)$ of the $T_{\text {OTN }}$ and $T_{\text {OTK }}$ fits was also used to filter measurements with excessive noise or where the flame may have moved out of the field of view.

\subsection{Atomic Emission of $\mathrm{Na}$ and $\mathrm{K}$ in a Flame}

This section introduces the processes by which sodium $(\mathrm{Na})$ and potassium $(\mathrm{K})$ in a flame may be perturbed to emit electromagnetic radiation. These species may be carried to the flame within salt-laden aerosols containing species such as sodium chloride $(\mathrm{NaCl})$ and potassium chloride $(\mathrm{KCl})$. As a salt-laden aerosol enters the combustion zone of a flame, its water component will begin to evaporate (Lajunen 2004; Alkemade et al. 1970). This leads to the nucleation of likely compounds (e.g., $\mathrm{NaCl}$ and $\mathrm{KCl}$ in the case of $\mathrm{NaCl}$ and $\mathrm{KCl}$ laden aerosols), which are expected to form solid or molten particles post dehydration (Lajunen 2004; Alkemade et al. 1970). It is possible for these nucleated salts to undergo intermediate reactions that form refractory oxides, fluorides, acids, or other intermediate compounds (Alkemade et al. 1970). In particular, newly formed $\mathrm{Mg}, \mathrm{Ca}$, and $\mathrm{Al}$ salts are known to react with $\mathrm{H}_{2} \mathrm{O}$ to form refractory oxides, which have boiling points higher than typical hydrocarbon flames (i.e., $>2300 \mathrm{~K}$ ) (Alkemade et al. 1970). These intermediate reactions generally depend on flare gas and aerosol composition and flame temperature (Alkemade et al. 1970).

If nucleated compounds have boiling point temperatures less than the flame temperature (i.e., $<2300 \mathrm{~K}$ ), they may sublime (or melt and evaporate) and transition into the gas phase (Lajunen 2004; Alkemade et al. 1970). The boiling point temperature of $\mathrm{Na}, \mathrm{NaCl}, \mathrm{K}$, and $\mathrm{KCl}$ are all less than typical hydrocarbon flame temperatures as shown in Table 2.2.

Table 2.2: Boiling point temperatures for $\mathrm{Na}, \mathrm{NaCl}, \mathrm{K}, \mathrm{KCl}$ (Lewis 2004).

\begin{tabular}{|l|l|l|l|l|}
\cline { 2 - 5 } \multicolumn{1}{c|}{} & $\mathbf{N a}$ & $\mathbf{N a C l}$ & $\mathbf{K}$ & $\mathbf{K C l}$ \\
\hline Boiling point temperature $[\mathrm{K}]$ & 1154 & 1710 & 1047 & $1773^{\mathrm{a}}$ \\
\hline
\end{tabular}

${ }^{\mathrm{a}}$ sublimation temperature 
After transitioning into the gas phase, $\mathrm{NaCl}_{\mathrm{g}}$ and $\mathrm{KCl}_{\mathrm{g}}$ may dissociate into free, neutrally charged atoms (e.g., $\mathrm{Na}_{\mathrm{g}}$ and $\mathrm{K}_{\mathrm{g}}$ ) (Lajunen 2004; Alkemade et al. 1970). The dissociation reaction for an $\mathrm{NaCl}_{\mathrm{g}}$ molecule is (Alkemade et al. 1970):

$$
N a C l_{g} \rightleftharpoons N a_{g}+C l_{g}
$$

Alkemade et al. (1970) states that when in chemical equilibrium, the dissociation and reverse association reactions are balanced and governed by the mass action law. The mass action law shows that dissociation is dependent on the concentration of products already present in the flame (Lajunen 2004; Alkemade et al. 1970). This means the dissociation reaction could be suppressed by the molar concentration of $\mathrm{Cl}_{\mathrm{g}}$ in the flame (e.g., $\mathrm{Cl}_{\mathrm{g}}$ may be sourced from $\mathrm{KCl}_{\mathrm{g}}$ if present in the flame).

Newly formed $\mathrm{Na}_{\mathrm{g}}$ atoms may experience re-association, additional chemical reactions, or ionization (Alkemade et al. 1970). The ionization of alkali metals is prevalent in flames due to their relatively low ionization energies; the degree of ionization of a flame species is proportional to flame temperature (Lajunen 2004; Alkemade et al. 1970). The ionization reaction for $\mathrm{Na}_{\mathrm{g}}$ is:

$$
N a_{g} \rightleftharpoons N a_{g}^{+}+e
$$

where $e$ is an ionized electron (Alkemade et al. 1970). Similar to dissociation, when in chemical equilibrium the ionization and recombination processes are balanced and governed by Saha's law (Lajunen 2004; Alkemade et al. 1970). Saha's law shows that a more readily ionized compound (e.g., $\mathrm{K}_{\mathrm{g}}$ ) may increase the concentration of $e$ in the flame, suppressing the ionization of $\mathrm{Na}_{\mathrm{g}}$ (Lajunen 2004; Alkemade et al. 1970).

A gaseous atomic species in a flame may gain or lose energy by inelastic collisions, chemical reactions, or radiative processes (Alkemade et al. 1982). When an atom gains or loses internal energy via radiative processes it transitions between two electronic states, each corresponding to a particular energy level and electron configuration (Alkemade et al. 1982). Ignoring multiple simultaneous electron movements, this means that a state transition involves the moving of a single electron from one orbital to another. An atom that loses energy via a radiative process will release electromagnetic radiation (i.e., a discrete packet of energy, or, a photon) (Alkemade et al. 1982). The wavenumber of 
radiation that is emitted due to the transition from a high-energy to low-energy quantum state (or electronic energy state) is defined by in Planck's equation:

$$
E_{i_{j}}^{\prime \prime}-E_{i_{j}}^{\prime}=h c \tilde{v}_{o, i_{j}} \cdot 10^{2}
$$

where $E$ is the energy $[\mathrm{J}]$ of the lower $\left({ }^{\prime}\right)$ and upper $\left({ }^{\prime \prime}\right)$ states involved with $j^{\text {th }}$ state transition of the $i^{\text {th }}$ species.

In this study, the combination of $(i)$ and $(j)$ subscripts defines an emission "line" and will be referred to as such; $\tilde{v}_{o}$ is the primary wavenumber $\left[\mathrm{cm}^{-1}\right]$ of the electromagnetic radiation emitted by the given line (Alkemade et al. 1982). In atomic emission spectroscopy literature, it is commonly understood that each atomic element has a unique set of electronic energy levels and therefore - according to Eq. (2.11) - has a unique set of emission lines (i.e., a unique spectrum). This means that observing an atomic emission signature of $\mathrm{Na}$ or $\mathrm{K}$ in a flare flame proves that species was present in the flare at the time of measurement.

\subsubsection{Persistent Atomic Emission Lines of $\mathrm{Na}$ and $\mathrm{K}$}

The National Institute of Standards and Technology (NIST) defines a persistent emission line as one that may be "observed over a broad range of experimental conditions" and which "usually include one or more resonance lines" (Kramida et al. 2020). A resonance line is produced when an energy state transition involves the weakest energy level (i.e., the ground level), and a "first" resonance line is produced when an energy state transition involves the two weakest energy levels (i.e., the ground level and the lowest excitable energy level, a.k.a. the first resonance levels) (Alkemade et al. 1982). The relatively low energy that is required for an atomic species to exist in its first resonance levels means that its first resonance lines are its most persistent emission lines (Alkemade et al. 1982).

The lowest excitable level of $\mathrm{Na}$ and $\mathrm{K}$ is actually a multiplet of two levels, which means there are two energy levels with faintly different energies (Alkemade et al. 1982). Electronic state transitions between first resonance levels of $\mathrm{Na}$ and $\mathrm{K}$ therefore produce a spectral doublet (i.e., a first resonance doublet) (Alkemade et al. 1982). The wavenumbers of the lines associated with the first resonance doublet of $\mathrm{Na}$ and $\mathrm{K}$ are shown in Table 2.3. 
Table 2.3: Location of the first resonance doublet of $\mathrm{Na}$ and $\mathrm{K}$ (Kramida et al. 2020).

\begin{tabular}{|c|c|c|c|c|}
\cline { 2 - 4 } \multicolumn{1}{c|}{} & \multicolumn{2}{c|}{ Na } & \multicolumn{2}{c|}{ K } \\
\hline \multirow{2}{*}{ Spectral location } & $16960 \mathrm{~cm}^{-1}$ & $16978 \mathrm{~cm}^{-1}$ & $12989 \mathrm{~cm}^{-1}$ & $13046 \mathrm{~cm}^{-1}$ \\
& $589.59 \mathrm{~nm}$ & $588.99 \mathrm{~nm}$ & $769.90 \mathrm{~nm}$ & $766.49 \mathrm{~nm}$ \\
\hline
\end{tabular}

\subsection{Local Thermodynamic Equilibrium and Electron Temperature}

A flame is said to be in physical equilibrium when the translational and internal energy distributions of all its constituent species may be described by a single equilibrium temperature $\left(T_{e q l}\right)$ (Alkemade et al. 1982). Chemical equilibrium exists when the degree of dissociation and ionization of all flame species adheres to the mass-action and Saha's laws (Alkemade et al. 1982). Physical and chemical equilibrium (i.e., thermal equilibrium) exists when $T_{\text {eql }}$ may also be used to describe the degree of dissociation and ionization of all flame species (Alkemade et al. 1982). Thermodynamic equilibrium exists when $T_{e q l}$ may be used to describe the spectral intensity of outgoing radiation defined by Plank's radiation law (Alkemade et al. 1982).

A flame can never be in true thermodynamic equilibrium because Planck's law will never be satisfied. In fact, Alkemade et al. (1982) states that a "[flame's] spectral radiance in most parts of the spectrum lies far below the Planck value at flame temperature". This is mainly caused by the non-adiabatic nature of a flame as it exchanges heat with its surroundings, secondary combustion reactions which may add heat, and strong concentration and temperature gradients (Lou et al. 2010). This is especially true in turbulent flames that entrain large volumes of fresh air (Alkemade et al. 1982). While these issues affect all components of flame equilibrium, they most strongly affect the density of its outgoing radiation field (i.e., Planck's blackbody equation) (Alkemade et al. 1982).

To model the atomic emission of species (i.e., $\mathrm{Na}$ and $\mathrm{K}$ ) within a flare flame, a state of local thermodynamic equilibrium is nevertheless assumed (Hanson et al. 2016; Wimberly 2015; Witte 2015; Potts 1992; Alkemade et al. 1982). This means that the volume of flame observed by the spectrometer systems is considered an isothermal "pocket" of the flame (Alkemade et al. 1982). This assumption is supported by Carinhana et al. (2008) who showed that the electronic temperature of sodium $\left(T_{\text {elec,Na }}\right)$ measured from an (assumed) 
adiabatic inner cone region of a steady state hydrocarbon-fueled flame adequately predicted its adiabatic flame temperature. Furthermore, Carinhana et al. (2008) stated that "under most burner conditions, sodium electronic temperature can be assigned as [the gas kinetic temperature] system temperature". This statement is extrapolated to include the electronic temperature of $\mathrm{K}$. This means that the mean $T_{g}$ (i.e., $\overline{T_{g}}$ ) calculated from a flare measurement is equal to the electronic temperature of $\mathrm{Nag}_{\mathrm{g}}$ and $\mathrm{K}_{\mathrm{g}}$ in the flame:

$$
T_{\text {eql }} \approx \bar{T}_{g} \approx T_{\text {elec }, N a} \approx T_{\text {elec }, K}
$$

\subsection{Modelling Atomic Emission Considering Self-Absorption and Induced \\ Emission}

A theoretical model for the spectral intensity of atomic emission that is measured by the spectrometer system is derived in this section considering self-absorption and induced emission. In brief, self-absorption occurs when a gaseous species absorbs electromagnetic radiation that itself emitted (Alkemade et al. 1982). It may be assumed that all selfabsorbed radiation within a flame is converted to translational kinetic energy (i.e., it is quenched) (Alkemade et al. 1982). Induced emission occurs when a species is perturbed to emission by an incoming radiation field (Alkemade et al. 1982). Induced emission is of the same direction, phase, and wavenumber of the incident radiation field (Alkemade et al. 1982).

To model these processes, consider a gaseous atomic species that is uniformly distributed throughout a flame such that the flame and its arbitrary species exist in local thermodynamic equilibrium. The flame is considered to be a gas slab and any far side incident radiation is ignored. The spectral radiance of an atomic emission source (i.e., atomic emission from a flare flame) at the location of the spectrometer system considering self-absorption is (Goldenstein et al. 2017):

$$
I(\tilde{v})=I_{B B-P}\left(\tilde{v}, T_{\text {elec }, i}\right) \varepsilon(\tilde{v})
$$

where $I_{B B-P}$ Planck's blackbody equation $\left[\mathrm{W} \mathrm{m} \mathrm{m}^{-2} \mathrm{sr}^{-1}\left(\mathrm{~cm}^{-1}\right)^{-1}\right], \varepsilon$ is emissivity [-]. 
An important characteristic of a spectroscopic measurement is the "smearing" (i.e., broadening) of narrow spectral features due to the mapping of monochromatic light onto its linear photodetector, which is spread over some non-zero region. The spread of monochromatic light is modeled via an instrument profile kernel, $\Phi_{I P}[-]$, whose functional form is a normalized Gaussian function (see Appendix A, Section A.2 for the functional form of the kernel used in this study). Fundamentally, the kernel models the spread of the source intensity at some center wavelength over its neighbouring wavelengths, ensuring total intensity is preserved. The full-width half-max $\left(\gamma_{I P}[-]\right)$ of the kernel is assumed to be the optical resolution of the spectrometer system $\left[\mathrm{cm}^{-1}\right]$, or, the minimum distance two narrow spectral features can be from each other and still be resolved.

In addition to instrumental broadening, source intensity is attenuated as its interacts with optical components in the spectrometer system such as lenses and mirrors. The attenuation is sensitivity to wavenumber and is effects are modeled by a transmission function. The broadened and attenuated light is then dispersed onto the spectrometer's linear photodetector, which converts the energy incident to each of its pixels into an arbitrary intensity unit, counts (i.e., counts pixel ${ }^{-1}$ ). A sensitivity function is required to convert the arbitrary intensity units into physical units (e.g., Watts $\mathrm{cm}^{-1}$ ) in order to produce a meaningful spectrum from photodetector data. The transmission and sensitivity functions of the spectrometer system can be simultaneously quantified by dividing the spectrometer readings of a known spectral intensity light source into its reference spectrum. The result is a relative spectral response curve, $\Psi$ [e.g., Watts pixel cm ${ }^{-1}$ counts $\left.^{-1}\right]$, which can then be multiplied with any raw spectrometer readings to produce a spectrum that has the correct "shape" (i.e., a spectrum with accurate relative intensities).

It is important to understand that $\Psi$ does not account for the cross sectional area of light that passes through the spectrometer system $\left[\mathrm{m}^{2}\right]$ nor the solid angle of light that the system measures. These parameters are spectrally invariant effectively scaling the intensity of a measured source and can be condensed into a single term, $C\left[\mathrm{~m}^{-2} \mathrm{sr}^{-1}\right]$, which is the optical conductance of the spectrometer. In functional form, the terms $\Phi_{I P}, \Psi$, and $C$ can be used to relate the raw spectrometer readings $\left(I_{\text {Raw }}\left[\right.\right.$ counts pixel $\left.\left.{ }^{-1}\right]\right)$ obtained during a remote 
flare measurement to the spectral radiance of the atomic source (i.e., the flare flame) at the location of the spectrometer system:

$$
I_{R a w}(\tilde{v}) \Psi(\tilde{v}) C=I(\tilde{v}) \Phi_{I P}\left(\tilde{v}_{o, i_{j}}-\tilde{v}, \gamma_{I P}\right)
$$

It is convenient to multiply $I_{\text {Raw }}$ with $\Psi$ as to replace these terms in Eq. (2.14) with a single intensity term, $I_{R e l}$ [a.u.], which defines accurate relative spectral intensities. The solid angle of flare flame that subtends the spectrometer system is assumed to vary continuously with the chaotic structure and orientation of turbulent flare flames. This means the value of $C$ is not known and that it can be brought over to the right hand side of Eq. (2.14). In addition to not knowing the solid angle, the observed intensity of the atomic source presumably depends on the stand-off distance (the distance between the spectrometer system and flare flame during a remote measurement). The effect of distance is unknown, but is assumed to be spectrally invariant and can thus be amalgamated into $C$. These modifications to Eq. (2.14) yield:

$$
I_{R e l}(\tilde{v})=C I(\tilde{v}) \Phi_{I P}\left(\tilde{v}_{o, i_{j}}-\tilde{v}, \gamma_{I P}\right)
$$

and substituting $I$ in Eq. (2.15) with Eq. (2.13) yields:

$$
I_{R e l}(\tilde{v})=C I_{B B-P}\left(\tilde{v}, T_{e l e c, i}\right) \varepsilon(\tilde{v}) \Phi_{I P}\left(\tilde{v}_{o, i_{j}}-\tilde{v}, \gamma_{I P}\right)
$$

Assuming the thermal radiator responsible for the atomic emission is in local thermodynamic equilibrium, then $\varepsilon$, absorptivity $(\alpha[-])$, and transmissivity $(\tau[-])$ are related via Kirchhoff's law (Alkemade et al. 1982).

$$
\varepsilon(\tilde{v})=\alpha(\tilde{v})=1-\tau(\tilde{v})=1-e^{-k_{i_{j}}(\tilde{v}) L}
$$

where $\tau$ may be rewritten using Beer's law, integrated along the path length through the flame ( $L[\mathrm{~cm}]$ ) (Alkemade et al. 1982). In Eq. (2.17), $k_{i_{j}}$ is the spectral absorption coefficient [(atom or molecule) $\mathrm{cm}^{-1}$ ] of the emission line whose functional form, as defined by Goldenstein et al. (2017), accounts for induced emission (See sections 2.1.1 and 
2.2.2. of Goldenstein et al. (2017) for the complete functional form of $k_{i_{j}}$-the partial form is shown in Appendix A Section A.2, and the requirements for induced emission are described by Alkemade et al. (1982)).

It is assumed that:

$$
k_{i_{j}}(\tilde{v}) L<<1
$$

which relies upon the volume fraction of the thermal radiator $\left(x_{i}[-]\right.$,contained within $\left.k_{i_{j}}\right)$ being $\lesssim 1 \times 10^{-6}$ when evaluating $k_{i_{j}}$ and $L$ using representative values (e.g., $L=0.4 \mathrm{~m}$, $T_{\text {elec, } i}=1900 \mathrm{~K}$, etc.). Although it is possible for the thermal radiator's volume fraction to be $\gtrsim 1 \times 10^{-6}$, it is irrelevant to this derivation because flame spectra were corrected by a relative calibration rather than an absolute calibration. Without an absolute calibration, the atomic emission model may only be used to assess the shape and location of suspected emissions signatures. Consequently, when fitting the atomic emission model to suspected emission signatures of $\mathrm{Na}$ and $\mathrm{K}, x_{i}$ was constrained to $1 \times 10^{-9}$ and the model was scaled via $C$. Details regarding the optimization of $C$ during least squared regression are presented in Chapter 4.

The assumption in Eq. (2.18) leads to an important simplification:

$$
1-e^{-k_{i_{j}}(\tilde{v}) L} \approx \kappa_{i_{j}}(\tilde{v}) L
$$

which, when ultimately applied to Eq. (2.16) yields:

$$
I_{R e l}(\tilde{v})=C I_{B B-P}\left(\tilde{v}, T_{\text {elec }, i}\right) k_{i_{j}}(\tilde{v}) L \Phi_{I P}\left(\tilde{v}_{o, i_{j}}-\tilde{v}, \gamma_{I P}\right)
$$

Rewriting $k_{i_{j}}$ and $\Phi_{I P}$ in Eq. (2.20) in terms of the first resonance doublet of the species $i$, where the subscript $(j)$ is replaced by the subscripts (1) and (2), representing the two lines of a first resonance doublet, yields :

$$
\begin{gathered}
I_{R e l}(\tilde{v})=C I_{B B-P}\left(\tilde{v}, T_{\text {elec }, i}\right) L\left[k_{i_{1}}(\tilde{v}) \Phi_{I P}\left(\tilde{v}_{o, i_{1}}-\tilde{v}, \gamma_{I P}\right)\right. \\
\left.+k_{i_{2}}(\tilde{v}) \Phi_{I P}\left(\tilde{v}_{o, i_{2}}-\tilde{v}, \gamma_{I P}\right)\right]
\end{gathered}
$$


It is now appropriate to assume that $I_{B B-P}$ is spectrally invariant within the narrow wavelength interval of a first resonance doublet, such that it can be approximated by a single blackbody intensity evaluated at the mean wavenumber of the first resonance doublet, $\left(\overline{I_{B B-P}}\right)$. Amalgamating $\overline{I_{B B-P}}$, and $L$ into $C$ to further simplify Eq. (2.21) yields:

$$
I_{R e l}(\tilde{v})=C\left[k_{i_{1}}(\tilde{v}) \Phi_{I P}\left(\tilde{v}_{o, i_{1}}-\tilde{v}, \gamma_{I P}\right)+k_{i_{2}}(\tilde{v}) \Phi_{I P}\left(\tilde{v}_{o, i_{2}}-\tilde{v}, \gamma_{I P}\right)\right]
$$

In Eq. (2.22), the Gaussian kernel of $\Phi_{I P}$ is convolved directly with the Voigt profiles of the line shape functions, $\phi_{i_{1}}$ and $\phi_{i_{2}}$ [cm], contained within $k_{i_{1}}$ and $k_{i_{2}}$, respectively (see Section 2.4 of Goldenstein et al. (2017) for details regarding the lineshape function). The spectral broadening effect due to $\Phi_{I P}$ and $\phi$ was compared (via their full-width half-max) to determine their relative importance when modelling the atomic emission of $\mathrm{Na}$ and $\mathrm{K}$. Table 2.4 shows the manufacturer predicted optical resolution (i.e., the $\gamma_{I B}$ of the spectrometer) near the first resonance doublet of $\mathrm{Na}$ and $\mathrm{K}$; and the estimated full-width half-maximum of the Doppler and collisional broadening $\left(\gamma_{D}\right.$ and $\gamma_{C}$, respectively, which make up $\phi$ ) for the first resonance doublet of $\mathrm{Na}$ and $\mathrm{K}$. The values for $\gamma_{D}$ and $\gamma_{C}$ were calculated according to Section 2.4 of Goldenstein et al. (2017), using a flame temperature of $1900 \mathrm{~K}$. Collisional broadening coefficients for $\mathrm{Na}$ and $\mathrm{K}$ with $\mathrm{N}_{2}$ were found in Table 8.1 in (Hanson et al. 2016)

Table 2.4: A comparison of the full-width half-maximum due to spectral broadening caused by the instrument, Doppler and collisional broadening that occurs during the radiative process.

\begin{tabular}{|l|l|l|l|}
\cline { 2 - 4 } \multicolumn{1}{c|}{} & Manufacturer Predicted $\gamma_{I B}\left[\mathrm{~cm}^{-1}\right]$ & $\gamma_{D}\left[\mathrm{~cm}^{-1}\right]$ & $\gamma_{C}{ }^{\mathrm{a}}\left[\mathrm{cm}^{-1}\right]$ \\
\hline Na first resonance doublet & 17.28 & 0.12 & 0.12 \\
\hline K first resonance doublet & 16.89 & 0.07 & 0.2 \\
\hline
\end{tabular}

${ }^{a}$ Assuming collisional broadening with $\mathrm{N}_{2}$ only.

Table 2.4 shows that $\gamma_{D}$ and $\gamma_{C}$ are approximately two orders of magnitude less than $\gamma_{I B}$ for both $\mathrm{Na}$ and $\mathrm{K}$. Therefore, the broadening due to $\Phi_{I P}$ is assumed to be much stronger than $\phi$, that is:

$$
\gamma_{I B} \gg \gamma_{D}, \gamma_{C}, \text { for } \mathrm{Na} \text { and } \mathrm{K}
$$

permitting the removal of $\phi_{i_{1}}$ and $\phi_{i_{2}}$ from $k_{i_{1}}$ and $k_{i_{2}}$ in Eq. (2.22), respectively. 
The functional form of $\Phi_{I P}$ (found in Appendix A, Section A.2) can now be substituted into Eq. (2.22) to produce:

$$
I_{R e l}(\tilde{v})=C\left[k_{i_{1}}(\tilde{v}) e^{-4 \ln 2 \frac{\left(\tilde{v}-\widetilde{v}_{o, i_{1}}\right)^{2}}{\gamma_{I P}{ }^{2}}}+k_{i_{2}}(\tilde{v}) e^{-4 \ln 2 \frac{\left(\tilde{v}-\widetilde{v}_{o, i_{2}}\right)^{2}}{\gamma_{I P}{ }^{2}}}\right]
$$

where the Gaussian profile heights (i.e., $\frac{2 \sqrt{2 \ln 2}}{\gamma_{I P} \sqrt{2 \pi}}$ ) were factored out of the square bracket and into $C$ (see Appendix A, Section A.2 for context regarding Gaussian peak height). The spectral intensity of atomic emission produced by the first resonance doublet of the $i^{\text {th }}$ species - as measured by the spectrometer system - was modeled via Eq. (2.24).

\subsection{Molecular Absorption Considering Induced Emission}

Modeling molecular absorption is important to this study because the $\mathrm{K}$ first resonance doublet $\left(12989 / 13047 \mathrm{~cm}^{-1}\right)$ is located within the wavenumber interval of the A band of molecular oxygen $\left(\mathrm{O}_{2}, \sim 12903\right.$ to $\left.13245 \mathrm{~cm}^{-1}\right)$ (Gordon et al. 2017; Ding et al. 2016). The ambient concentration of $\mathrm{O}_{2}$ in Earth's lower atmosphere is $\sim 21 \%$ by volume meaning that electromagnetic radiation emitted from flare flames will interact with (i.e., be absorbed by) $\mathrm{O}_{2}$ prior to being measured by the spectrometer system. The spectral molecular absorption profile of the A band is due to a cluster of lines that belong to a band system, produced by "electronic transitions [that] are accompanied by rotational-vibrational transitions" (Goody and Yung 1995).

Consider that gaseous $\mathrm{O}_{2}$ is uniformly distributed among the atmosphere in-between the flare and spectrometer system during a flare measurement. The volume of $\mathrm{O}_{2}$ observed by the spectrometer is considered a gas slab that exists in local thermodynamic equilibrium, with flare flame radiation incident to its far side. The spectral radiance of flare flame radiation after transecting the gas slab is defined as the transmitted intensity ( $I_{t}$ [W m$\left.\left.{ }^{-2} \mathrm{sr}^{-1}\left(\mathrm{~cm}^{-1}\right)^{-1}\right]\right)$ whereas the spectral radiance of flare flame radiation prior to entering the gas slab is defined as the incident intensity $\left(I_{o}\left[\mathrm{~W} \mathrm{~m}{ }^{-2} \mathrm{sr}^{-1}\left(\mathrm{~cm}^{-1}\right)^{-1}\right]\right) . I_{t}$ and $I_{o}$ are related through Beer's law, integrated along gas slab thickens, $(L[\mathrm{~cm}])$, which is equal to the stand-off distance (Alkemade et al. 1982): 


$$
I_{t}(\tilde{v})=I_{o}(\tilde{v}) \sum_{j=1}^{\infty} e^{-k_{i_{j}}(\tilde{v}) L}
$$

where $k_{i_{j}}$ is the spectral absorption coefficient of a single molecular absorption line $\left[\mathrm{cm}^{-1}\right]$. The $k_{i_{j}}$ for molecular lines differs from atomic lines by its linestrength term only, whose functional form is presented in section 2.2.1 in Goldenstein et al. (2017).

The transmitted intensity in Eq. (2.25) is the spectral radiance of flare flame emission at the spectrometer system's location. Similar to Eq. (2.15) in the atomic emission model derivation, this transmitted intensity is related to raw spectrometer readings as such:

$$
\begin{gathered}
I_{R a w}(\tilde{v}) \Psi(\tilde{v}) C=I_{t}(\tilde{v}) \Phi_{I P}\left(\tilde{v}_{o, i_{j}}-\tilde{v}, \gamma_{I P}\right) \\
I_{R e l}(\tilde{v})=C I_{t}(\tilde{v}) \Phi_{I P}\left(\tilde{v}_{o, i_{j}}-\tilde{v}, \gamma_{I P}\right)
\end{gathered}
$$

and introducing the function form of $I_{t}$ into Eq. (2.26) yields:

$$
I_{R e l}(\tilde{v})=C I_{o}(\tilde{v}) \sum_{j=1}^{\infty} e^{-k_{i_{j}}(\tilde{v}) L} \Phi_{I P}\left(\tilde{v}_{o, i_{j}}-\tilde{v}, \gamma_{I P}\right)
$$

The effects of instrument broadening on $I_{o}$ are ignored since the spectral shape of $I_{o}$ changes gradually, like a blackbody curve. Therefore, the result of multiplying $C$ with $I_{O}$ can be approximated by interpolating the background intensities of the molecular absorption features in $I_{R e l}$. Replacing $\left(C \times I_{o}\right)$ in Eq. (2.27) with the interpolated background term ( $I_{\text {interp }}$ [a.u.]), and introducing the functional form of $\Phi_{I P}$ yields:

$$
I_{R e l}(\tilde{v})=D I_{\text {interp }}(\tilde{v}) \sum_{j=1}^{\infty} e^{-k_{i_{j}}(\tilde{v}) L} e^{-4 \ln 2 \frac{\left(\tilde{v}-\tilde{v}_{o, i}\right)^{2}}{\gamma_{I P}}}
$$

where the Gaussian profile height is factored out of the summation and into the out-front term, $D$.

The spectral broadening effect of $\Phi_{I P}$ and $\phi$ on the molecular absorption model (Eq. (2.28)) was compared (similar to the atomic emission model). The $\gamma_{D}$ and $\gamma_{C}$ were 
calculated for each line in the $\mathrm{O}_{2} \mathrm{~A}$ band using values from the high-resolution transmission molecular absorption database (HITRAN), assuming a temperature of $1900 \mathrm{~K}$ (Gordon et al. 2017). For each line in the $\mathrm{O}_{2} \mathrm{~A}$ band, the values of $\gamma_{D}$ and $\gamma_{C}$ are three orders of magnitude less than the $\gamma_{I P}$ predicted by the manufacturer. This means that, similar to atomic emission, broadening due to $\Phi_{I P}$ is much stronger than $\phi$, or:

$$
\gamma_{I B} \gg \gamma_{D}, \gamma_{C} \text {, for all lines in the } \mathrm{A} \text { band of } \mathrm{O}_{2}
$$

permitting the removal of $\phi_{i_{j}}$ from $k_{i_{j}}$ in Eq.(2.28). In any case, the spectral profile of molecular absorption of the $\mathrm{O}_{2} \mathrm{~A}$ band - as measured by the spectrometer system - is modeled via Eq.(2.28). 


\section{Chapter 3 Experimental Setup and Calibrations}

Two customized spectrometer systems, a "narrow-band" and a "broad-band" version, were used to remotely measure the radiative emission of upstream oil and gas (UOG) flare flames. These systems were identical except for the internal configuration of their spectrometer. The diffraction grating within the narrow-band spectrometer was configured to measure the atomic emission of sodium $(\mathrm{Na})$ at 588.99 / $589.59 \mathrm{~nm}$ and had a spectral range of 485 to $605 \mathrm{~nm}$ with an average spectral resolution of $0.089 \mathrm{~nm}$. The diffraction grating within the broad-band spectrometer was configured to measure continuous emission of soot incandescence and had a spectral range of 500 to $900 \mathrm{~nm}$ with an average spectral resolution of $0.33 \mathrm{~nm}$. This range also permitted detection of the atomic emission of potassium $(\mathrm{K})$ at $766.48 / 769.89 \mathrm{~nm}$. A technical explanation of how the spectrometer systems function, the process behind measuring the radiative emission of an UOG flare, and details regarding intensity and wavelength calibrations, and estimation of minimum detection limits are presented in this chapter.

\subsection{The Spectrometer Systems and their Components}

The narrow-band and broad-band spectrometer setups shared the same components as illustrated in Figure 3.1. Each system included a lens assembly that focused light into the slit of a portable spectrometer (Ocean Flame-S spectrometer, models FLMS14908 (narrow-band) and FLMS14909 (broad-band)). To capture the radiative emission of a flare flame from a distance, each apparatus had a single $100 \mathrm{~mm}$ in diameter plano-convex lens with a $400 \mathrm{~mm}$ focal length (Edmund Optics stock \# 27503) that was used to focus light onto the slit of the spectrometer. The plano-convex lens was mounted inside an assembly of adjustable lens tubes (custom/Thorlabs) which stepped down in diameter from $100 \mathrm{~mm}$ at the lens to $12.5 \mathrm{~mm}$ at the spectrometer. The spectrometers were attached to the narrow end of the lens tube assemblies via a compact lockable $\mathrm{X}-\mathrm{Y}$ translation stage (Newport model M-DS25-XY). Each spectrometer was mounted such that its entrance slit was coplanar with the focal plane of the lens and the translation stage was used to align the entrance slit so that it was coincident with the optical axis. Finally, each apparatus was attached to a Manfrotto quick release plate which allowed it to be quickly and easily 
snapped into an articulating 3-axis geared tripod head (Manfrotto model 410) and screwed onto a lightweight tripod (Manfrotto model MTO55CXPRO3) for positioning and aiming in the field.

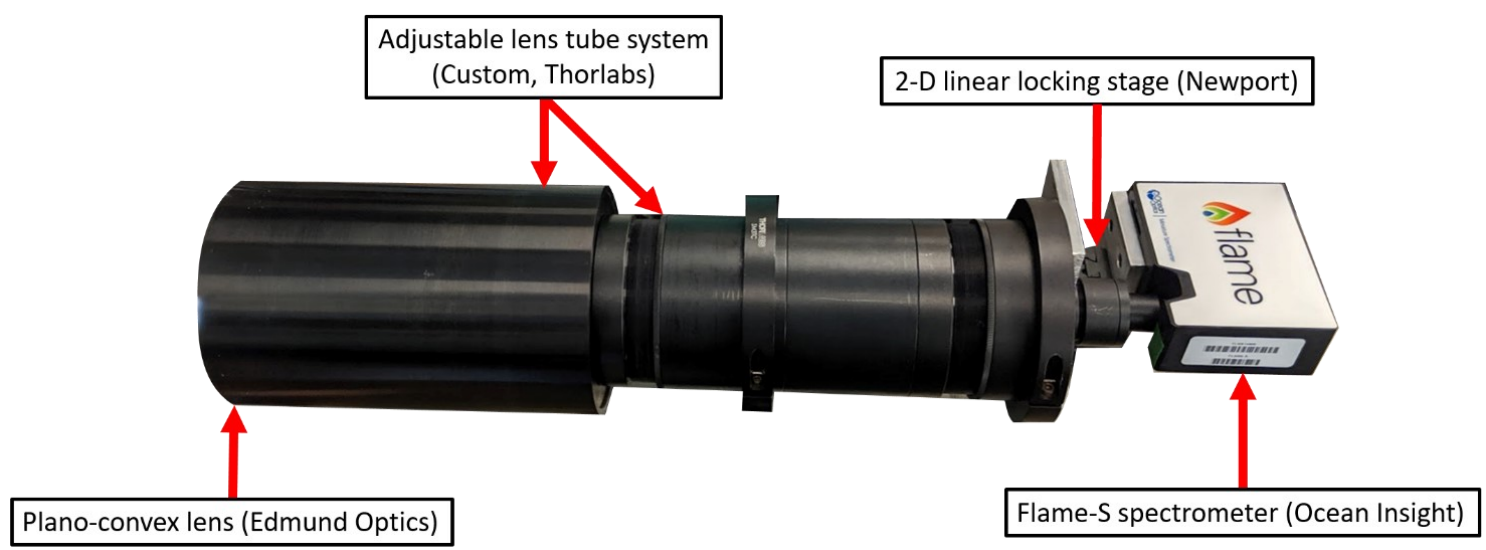

Figure 3.1: Image of the spectrometer-lens system.

\subsubsection{Operation of the Slit Spectrometer}

The spectrometers used in the narrow-band and broad-band systems were Ocean Insight (formerly Ocean Optics) Flame-S spectrometers. These small and lightweight devices $(89.1 \times 63.3 \times 34.4 \mathrm{~mm}, 265 \mathrm{~g})$ had three main components (Figure 3.2): the interchangeable entrance slit, the preconfigured optical bench, and the linear array chargecoupled device detector (i.e., the linear detector).

The interchangeable entrance slit acts as an entrance aperture for incoming polychromatic light. These $1 \mathrm{~mm}$ tall, rectangular slits (with varying widths) are oriented such that when the spectrometer is placed on a flat horizontal surface, the width dimension lies on the horizontal plane. The slit was integrated within a bulkhead that was secured to the body of the spectrometer with two screws. A $0.05 \times 1 \mathrm{~mm}$ (width $\times$ height) entrance slit was used with the narrow-band spectrometer which resulted in a (manufacturer predicted) $0.6 \mathrm{~nm}$ optical resolution (which is the full width half max of its Gaussian dispersion profile). The broad-band spectrometer used either a $0.025 \times 1 \mathrm{~mm}$ or a $0.01 \times 1 \mathrm{~mm}$ entrance slit which resulted in a (manufacturer predicted) optical resolutions of $1.4 \mathrm{~mm}$ or 
$1 \mathrm{~nm}$, respectively. The slits were selected to optimize the intensity of typical UOG flare flames and preserve the sharpness of its spectral features.

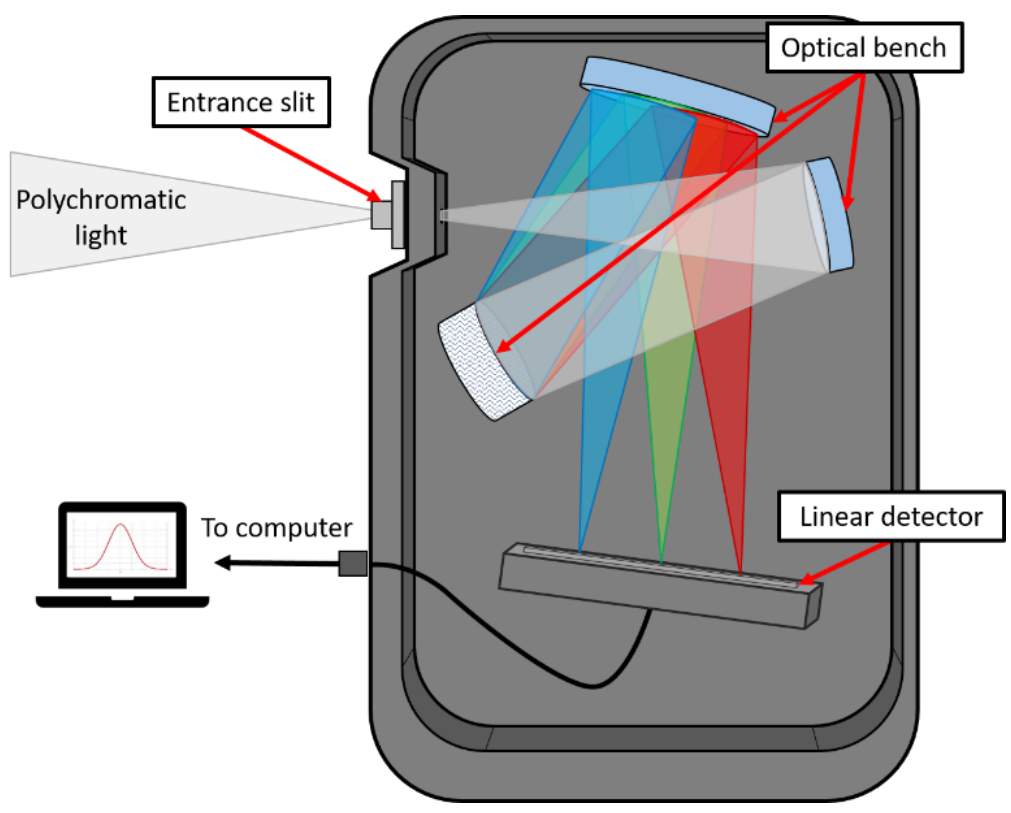

Figure 3.2: The 3 main components of the Flame-S spectrometer: the interchangeable entrance slit, the optical bench, and the linear detector.

The optical bench has a Czerny-Turnery configuration, which included two collimating mirrors $(0.22$ numerical aperture, or an $\mathrm{f}$-number $\sim 2.3)$ and one diffraction grating. The first mirror accepted polychromatic light from the entrance slit and collimated it onto the grating. The polychromatic light incident to the diffraction grating was reflected as monochromatic light. The light leaving the grating was chromatically separated such that each wavelength has a unique reflection angle. The second collimating mirror accepted these uniquely angled light beams and focused them onto the detector at unique locations. This is illustrated in Figure 3.3. 

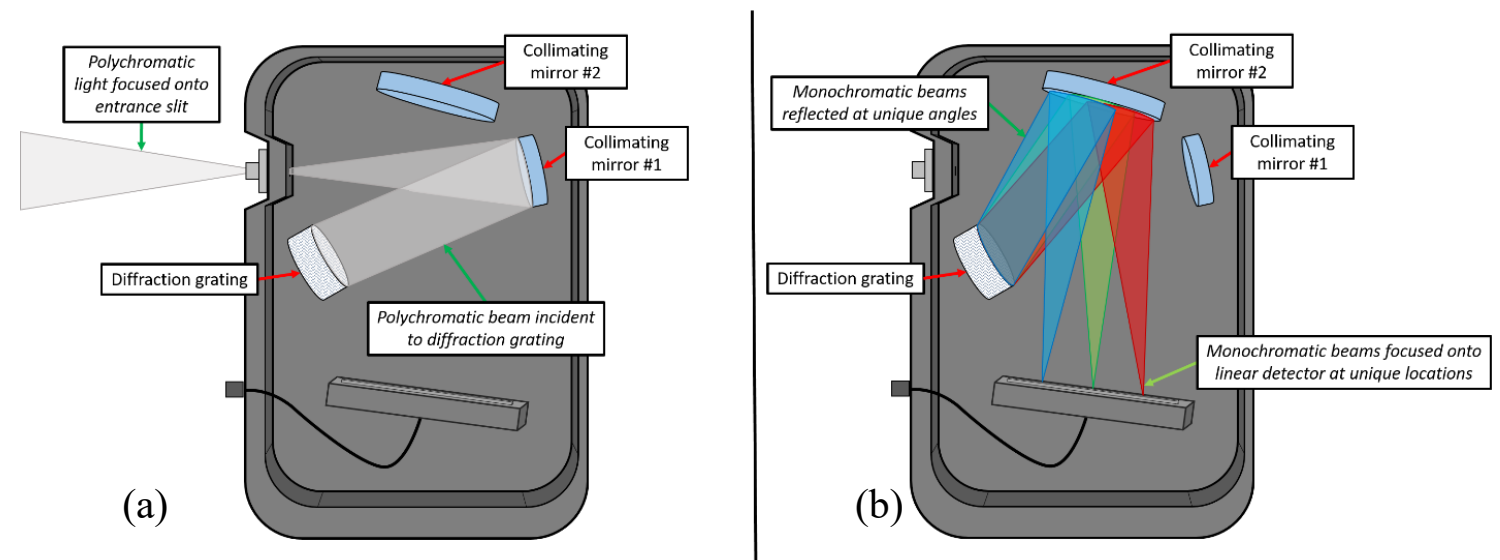

Figure 3.3: A diagram of the spectrometer measuring the monochromatic components of polychromatic light: (a) polychromatic light incident to diffraction grating, (b) monochromatic light reflected from DG onto linear detector.

The linear detector was a Sony ILX511B detector with 2048, $0.014 \mathrm{~mm}$ wide $\times 0.2 \mathrm{~mm}$ tall pixels oriented in a linear array. The raw intensity of monochromatic light incident to each pixel was measured in the arbitrary units of "counts" at 16-bit resolution. The linear detector was capable of reaching integration times as low as $1 \mathrm{msec}$. The spectrometer communicated and received power through a single USB connection and was typically controlled via a Panasonic Toughbook laptop running the OceanView 1.6.7 (Lite) software.

\subsubsection{System Field of View}

The field of view (FOV) of the spectrometer system represents the size of the region within the flare flame that was observed during a measurement. The FOV may be estimated using principal rays which are traced from entrance slit boundaries through the optical axis of the plano-convex lens and onto the flare flame being measuring. This means that the FOV is sensitive to measurement distance, the focal length of the lens, and the entrance slit dimensions. The following equation may be used to determine FOV dimensions:

$$
F O V_{H, W}=d \tan \left(\tan ^{-1} \frac{S_{H, W}}{f}\right)
$$

where $d$ is stand-off distance [m] (i.e., the distance between the spectrometer and the flame), $f$ is focal length of the plano-convex lens $[\mathrm{mm}]$, and $F O V_{H, W}$ and $s_{H, W}$ are the dimensions of the FOV $[\mathrm{m}]$ and the entrance slit [mm], where the subscripts indicated the height $(H)$ or width $(W)$, respectively. For the widest slit used during field measurements 
(i.e., the $0.05 \mathrm{~mm}$ wide slit used with the narrow-band spectrometer), the maximum extent of flame imaged at a typical distance of $125 \mathrm{~m}$ was estimated to be approximately $0.313 \mathrm{~m}$ tall by $0.016 \mathrm{~m}$ wide, as depicted in Figure 3.4. For the narrowest slit (i.e., the $0.01 \mathrm{~mm}$ slit used with the broad-band spectrometer), the estimated FOV was approximately $0.313 \mathrm{~m}$ tall by $0.003 \mathrm{~m}$ wide. In each case, the field system imaged a comparatively small region of the typically $>2-5 \mathrm{~m}$ long flare flame, which increased the chances of completely filling the spectrometer with emission signal from the flame, rather than a mix of flame and background sky, as the flame moved.

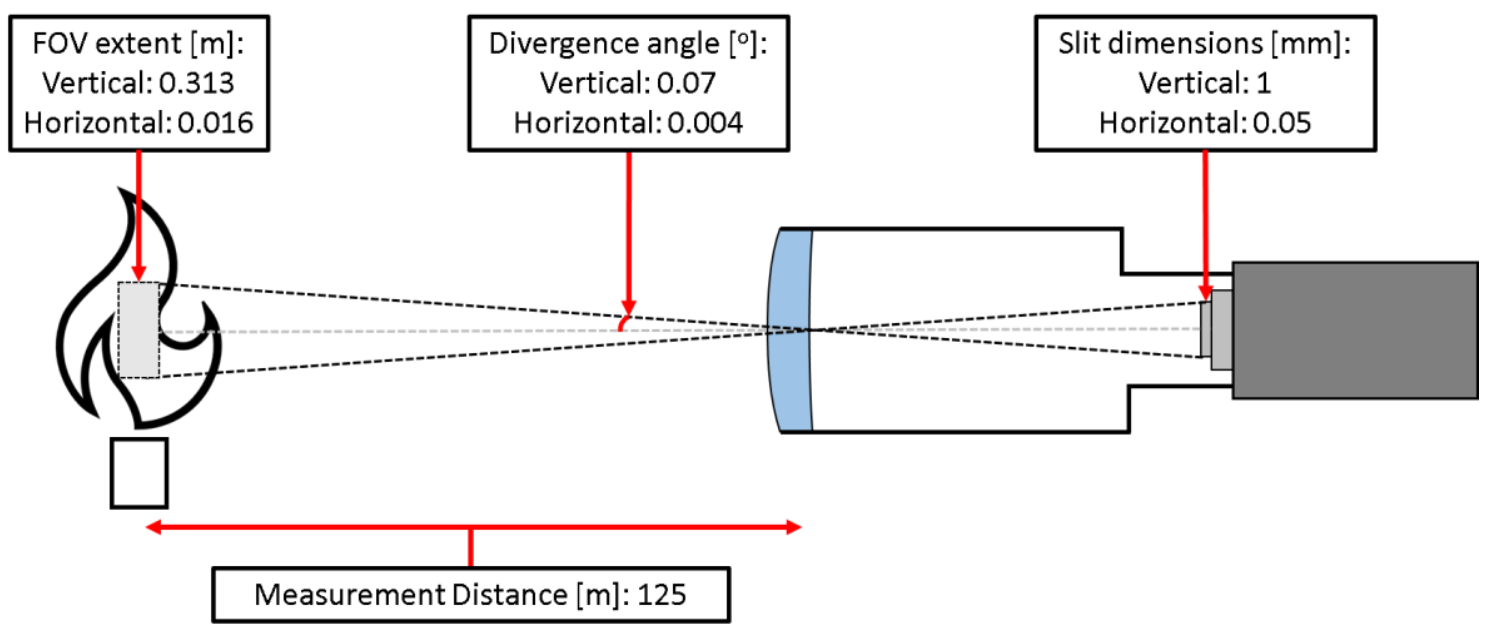

Figure 3.4: Depiction of the FOV calculation for the narrow-band system.*Diagram not to scale.

\subsubsection{Fine Focusing of the Spectrometer's Entrance Slit to the Focal Point of Plano-Convex Lens}

The narrow-band and broad-band spectrometers were fine focused prior to deployment in field measurement campaigns by maximizing the received intensity from a light source at a distance of $125 \mathrm{~m}$ (corresponding to the typical distance between a flare flame and the spectrometer during field measurements). As shown in Figure 3.5, this was achieved using an outdoor light with a $\sim 0.4 \mathrm{~m}$ in diameter spherical enclosure, which approximated a uniform light source of consistent spectral radiance. The distance to the light was measured using a laser range finder (Athlon model 502003-AD03). Fine focus measurements were completed at night such that the light was measured against dark background. 
During the fine focus procedure, the aim of the spectrometer system was first adjusted while simultaneously monitoring the spectra that were being previewed by the OceanView program via the Toughbook laptop. The aim was locked in place when the spectra appeared to reach maximum intensity. Then the fine position of the spectrometer's entrance slit was adjusted within the focal plane using the linear stage, while its position along the optical axis was adjusted by screwing or unscrewing the threaded lens tube. The positional adjustments of the entrance slit that resulted in the most intense spectra were locked in place. The entire fine focus procedure, starting from aiming the system at the light source, was repeated iteratively until the intensity of the observed spectra was maximized.

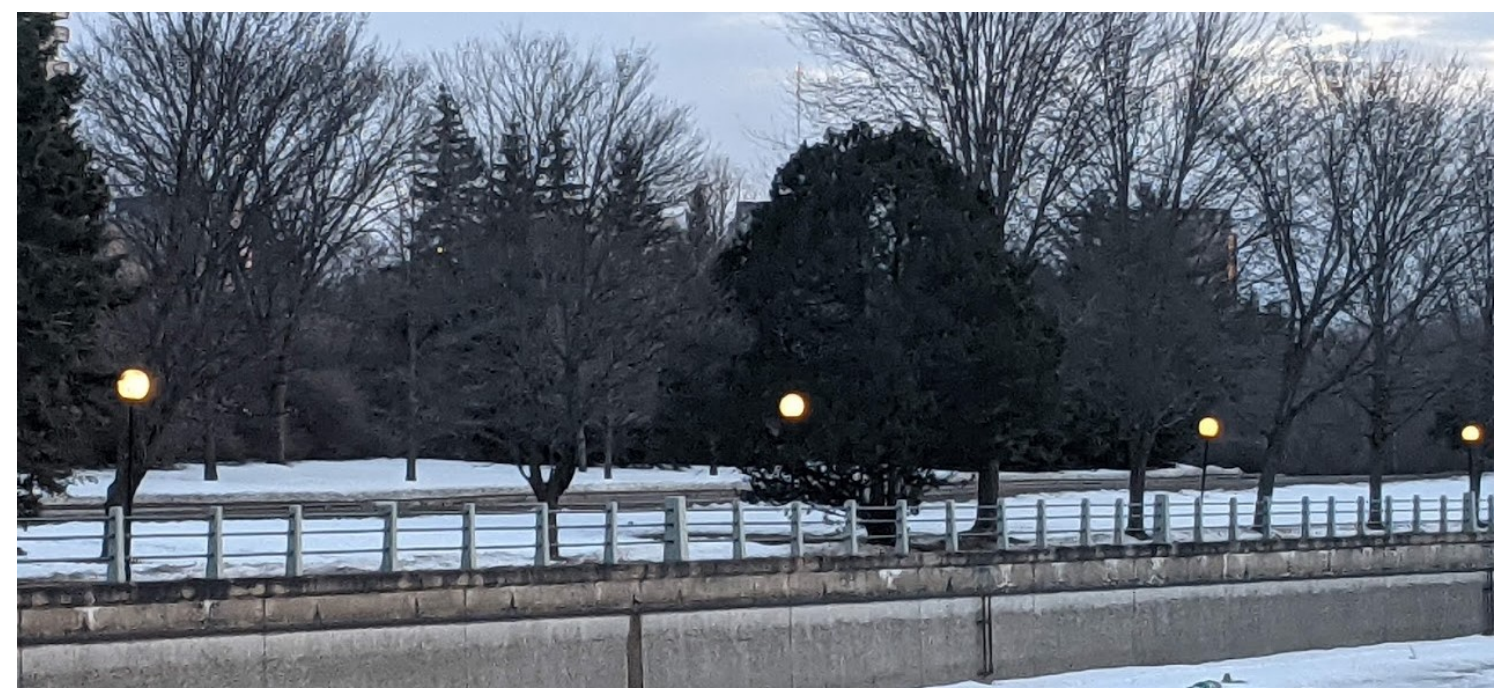

Figure 3.5: Example of the type of type of streetlight that was used to fine focus the spectrometer system.

\subsection{Relative Spectral Response Calibrations}

The relative spectral response of each spectrometer was calibrated such that the raw measured intensities [counts pixel ${ }^{-1}$ ] which make up flare spectra could be transformed into arbitrary intensity units [a.u.] proportional to spectral radiance $\left[\mathrm{W} \mathrm{m}^{-2} \mathrm{sr}^{-1}\left(\mathrm{~nm}^{-1}\right)^{-1}\right]$. The calibration of the broad-band spectrometer was performed twice, once with each slit that was used during field measurements (i.e., for the $0.025 \mathrm{~mm}$ and $0.01 \mathrm{~mm}$ wide slits).

A $135 \mathrm{~mm}$ diameter, spectralon coated integration sphere (Labsphere model \# CSTM-3PGPS-053-SL) illuminated with a $100 \mathrm{~W}$ NIST traceable tungsten-halogen lamp was used as a calibration source. The output spectral radiance of the integration sphere was provided 
by the manufacturer and was obtained using NIST traceable equipment and methods. During calibration, the spectrometer system was aligned along the axis of the integration sphere's $63.5 \mathrm{~mm}$ diameter exit port and positioned such that its plano-convex lens was approximately $25 \mathrm{~mm}$ away from the sphere's exit plane. Figure 3.6 shows the calibration system without and with the spectrometer in place. Not shown in Figure 3.6 is the thick plastic sheet that was used to bridge the gap between the spectrometer system and the integrating sphere to prevent stray light from entering the lens during a calibration.
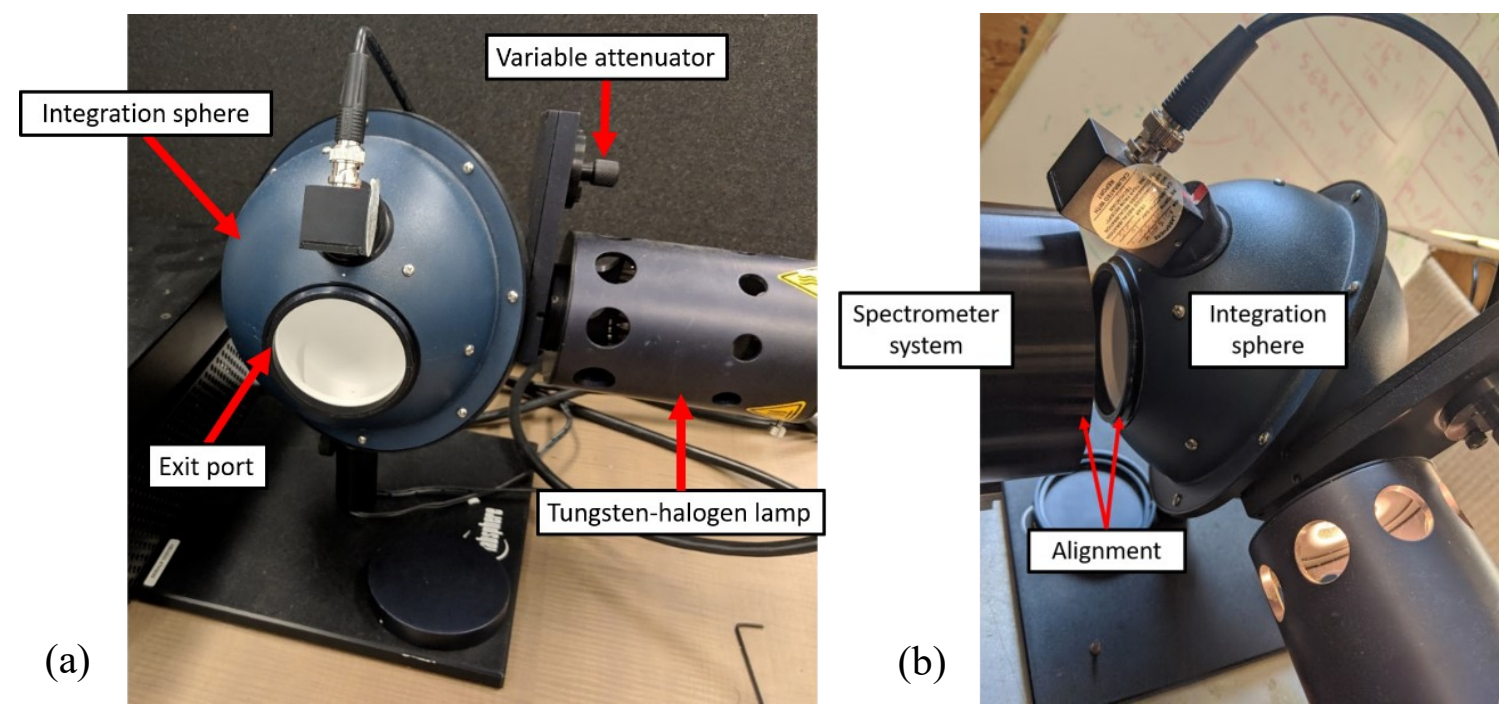

Figure 3.6: (a) A labelled diagram of the system used to calibrate the relative wavelength response of the spectrometers. (b) An example of the alignment between the spectrometer system and the integration sphere when performing a relative spectral response calibration. * Not shown is the thick plastic sheet that was used to bridge the gap between the spectrometer system and the sphere to prevent stray light from entering the lens during a calibration.

After the lamp was powered up with the variable attenuator opened for approximately fifteen minutes to allow the system to warm up, spectrometer readings were recorded at three different brightness levels, approximately 25,50 , and $75 \%$ of the saturation limit of the spectrometer's linear detector. The brightness level was adjusted using the variable attenuator located between the integrating sphere and the tungsten-halogen lamp (see Figure 3.6 (a)). These brightness levels were chosen to capture any non-linearities in the linear detector as it became saturated with light. After the three spectrometer readings were recorded at the various brightness levels, a fourth spectrometer reading was recorded with the lamp turned off. The fourth reading quantified the background intensity that was present during the spectrometer readings. It was important the four spectrometer readings 
(i.e., a set of spectrometer readings) did not depend on the internal alignment of the spectrometer system because the spectrometer system was partially disassembled and reassembled when preparing for travel / field measurements. This was accounted for by performing multiple sets of spectrometer readings where, between each set, the spectrometer system was partially disassembled, its entrance slit was perturbed, and the spectrometer system was reassembled.

In total, nine sets of spectrometer readings were recorded for each spectrometer system and slit, which were used to produce average measured intensity curves (with background levels subtracted) at the three different intensity levels (i.e., 25, 50, and $75 \%$ of the saturation limit of the detector). These curves are called "saturation curves", and are plotted in Figure 3.7 (a) for the narrow-band spectrometer and in Figure 3.7 (b) for the broad-band spectrometer with both the $0.01 \mathrm{~mm}$ wide slit (solid lines) and the $0.025 \mathrm{~mm}$ wide slit (dashed lines).

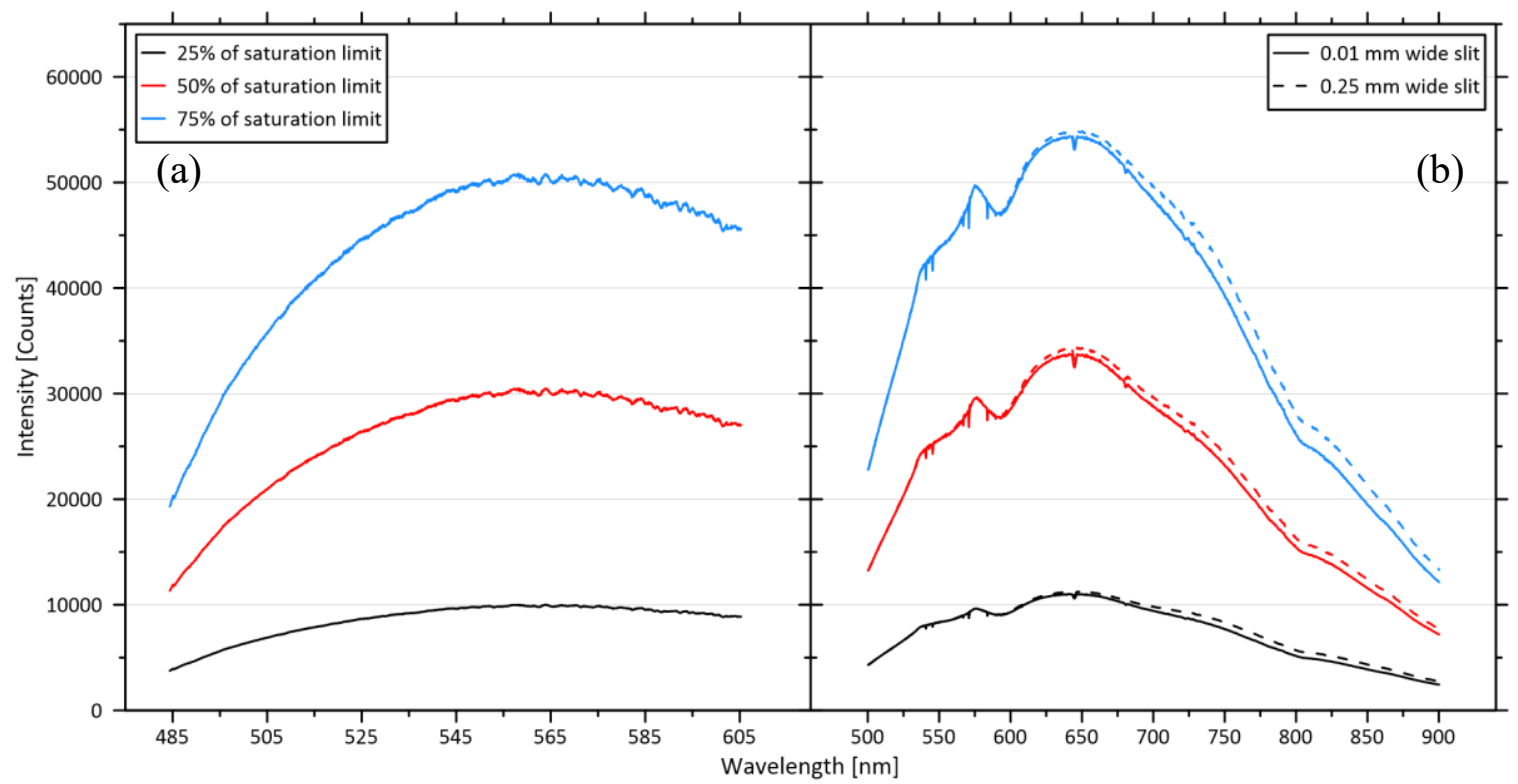

Figure 3.7: Mean spectrometer readings of the calibrated integration sphere at approximately 25, 50, and $75 \%$ of the saturation limit of the spectrometer's linear detector for the (a) narrow-band spectrometer and (b) broad-band spectrometer with the $0.01 \mathrm{~mm}$ wide entrance slit (solid lines) and the $0.025 \mathrm{~mm}$ wide slit (dotted lines).

Dividing each of these saturation curves by the calibrated reference spectra for the integrating sphere yielded the desired relative spectral response calibrations as plotted in 
Figure 3.8. These relative response curves were normalized by the intensities at the wavelengths indicated in Table 3.1 to facilitate direct visual comparison of their shapes.

Table 3.1: The wavelength used to normalize relative spectral response curves.

\begin{tabular}{|l|l|}
\hline Spectrometer & $\begin{array}{l}\text { Wavelength used to Normalize } \\
\text { Relative Spectral Response Curves }\end{array}$ \\
\hline Narrow-band & $606 \mathrm{~nm}\left(16502 \mathrm{~cm}^{-1}\right)$ \\
\hline Broad-band & $900 \mathrm{~nm}\left(11111 \mathrm{~cm}^{-1}\right)$ \\
\hline
\end{tabular}

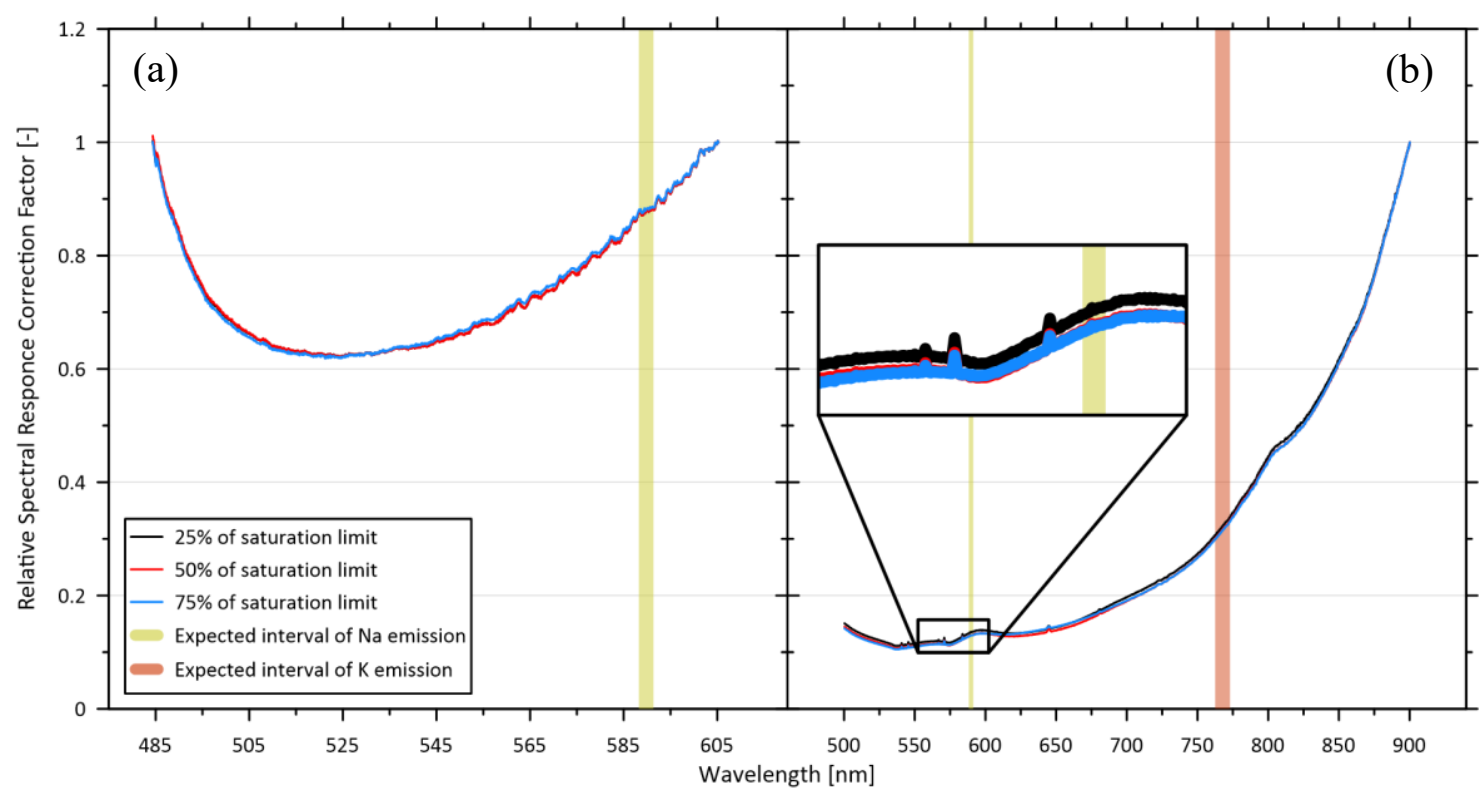

Figure 3.8: Relative spectral response curves (corresponding to approximately 25,50 , and $75 \%$ of the saturation limit of the spectrometer's linear detector). (a) Curves for the narrow-band spectrometer normalized by the intensity at $606 \mathrm{~nm}$. (b) Curves for the broad-band spectrometer with a $0.01 \mathrm{~mm}$ wide entrance slit normalized by the intensity at $900 \mathrm{~nm}$. Similar curves were obtained for the $0.025 \mathrm{~mm}$ slit but are not shown here.

Although the relative response curves at different intensities were closely aligned, there were some slight differences as illustrated in the magnification window in Figure 3.8 (b). When analyzing flare spectra acquired during field measurements, these small deviations were considered by using linear interpolation to estimate a unique relative response curve for flare spectra whose intensities were not at exactly 25,50 , or $75 \%$ of the saturation limit. For example, the background-subtracted intensity of each data point in a flare spectrum was linearly interpolated between the "saturation curves" of Figure 3.7 and the relative response curves in Figure 3.8 to estimate intensity-specific calibration factors. Together, these calibration factors form the "curve" used to calibrate the relative intensity of the 
measured flare spectrum. This process is shown by example in Chapter 4 where data processing techniques are discussed in detail.

\subsection{Assessment of Wavelength Shift}

If the FOV of the spectrometer system is filled asymmetrically or at an off-axis angle relative to its dispersion plane, aberrations (e.g., coma and/or astigmatism) in the optical bench can skew or shift the profiles of observed spectral features (Scheeline 2017; Herschel Space Observatory 2013; Goldstein and Walters 1976). This is a concern for field measurements of flares, since the turbulent nature of open atmosphere flare flames means the position of the flame is unpredictable and always changing. This increases the likelihood of asymmetrically filling of the spectrometer system's FOV, which could introduce wavelength shift or variation in the broadening of spectral features to the measured data.

The range of possible wavelength shifts was experimentally assessed using two UVP PENRAY lamps, one filled with Neon ( $\mathrm{Ne}$ ) gas and one filled with Krypton (Kr) gas, which produced known emission lines at precise wavelengths. The Ne lamp was used with the narrow-band spectrometer because it produced a dense cluster of emission lines near $590 \mathrm{~nm}$ (i.e., near the Na first resonance doublet). The Kr lamp was used to test the broadband system since its emission lines were spread throughout the visible/near-infrared wavelength range (500 to $800 \mathrm{~nm}$ ). Both lamps were $10 \mathrm{~mm}$ in diameter and $54 \mathrm{~mm}$ long (see Figure 3.9). Reference spectra that specified the known spectral peak locations for each lamp were found in the PEN-RAY Rare Gas Lamp Spectra document by Kaye (1997).

The wavelength shift assessment was performed on the narrow-band system with the $0.05 \mathrm{~mm}$ wide entrance slit and the broad-band system using the $0.01 \mathrm{~mm}$ wide entrance slit. The $0.01 \mathrm{~mm}$ wide entrance slit was chosen because it was used in $>75 \%$ of the broadband flare measurements. Spectrometer readings were acquired with the lamp in various placements (i.e., locations and orientations) with respect to the dispersion plane of the spectrometer. The lamp was held by an articulating arm and positioned it $\sim 25 \mathrm{~mm}$ away from the plano-convex lens of the spectrometer system. The lamp was oriented such that its length was perpendicular to the dispersion plane of the spectrometer and the arm was 
locked in place. The arm holding the lamp was attached to a linear translation stage that was aligned parallel to the dispersion plane of the spectrometer. This arrangement permitted the lamp to be reliably translated across face of the plano-convex lens and is shown in Figure 3.9.

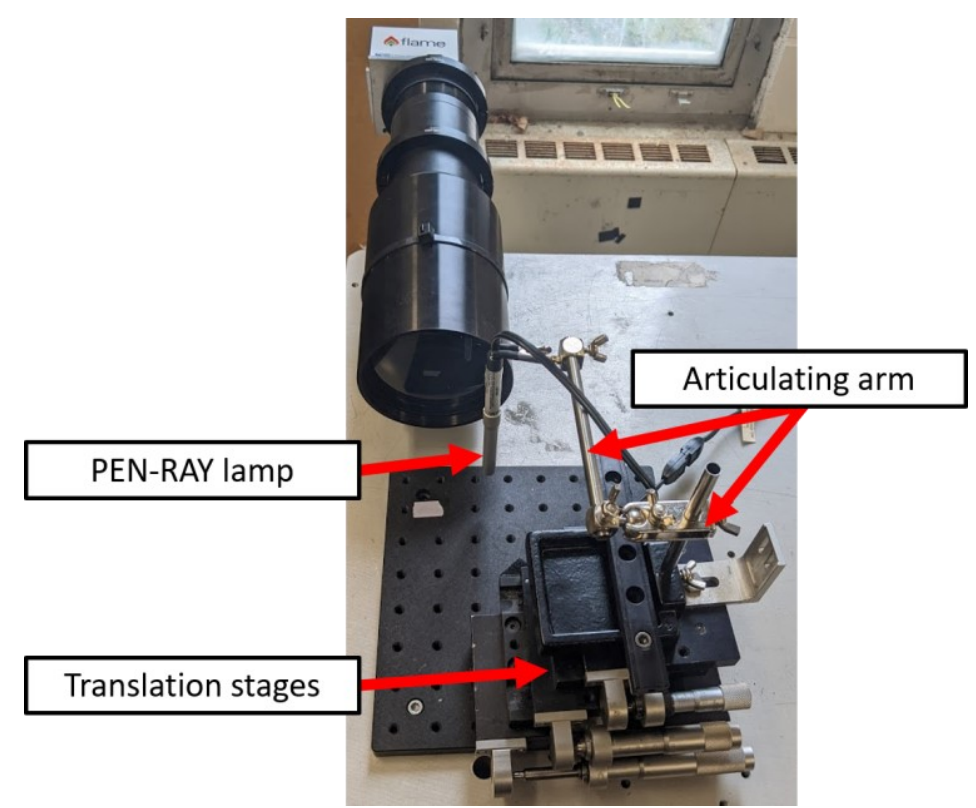

Figure 3.9: One of the UVP PEN-RAY lamps that were used to assess the shift and skewness of measured spectral peaks, caused by the off-axis illumination of the spectrometer's optical bench.

A set of spectrometer readings were acquired at $5 \mathrm{~mm}$ translation increments between -40 and $+40 \mathrm{~mm}$ of the plano-convex lens' centerline. At each location, the integration time of the spectrometer was adjusted to account for the reduction in light throughput as the lamp was translated away from the centerline, and then $\sim 30$ s of spectrometer readings were recorded. The lamp was then turned off and background spectrometer readings were acquired. The lamp was then turned back and translated to the next measurement location. During analysis, the average spectrometer reading with the lamp on minus the average reading with the lamp off for each location constituted a measurement point. This entire process was repeated three times such that three sets of spectrometer readings were acquired. Between the first and second set of readings, the spectrometer system was partially disassembled, its entrance slit was perturbed, and the spectrometer system was reassembled. For the third set of readings, the spectrometer system was rotated $90^{\circ}$ and the lamp was translated perpendicular the dispersion plane of the spectrometer. 


\subsubsection{Calculating Wavelength Shift Intervals}

To assess the wavelength shift of each spectrometer system, several isolated and easily identified emission lines were selected from the $\mathrm{Ne}$ and $\mathrm{Kr}$ lamp reference spectra found in Kaye (1997) as summarized in Table 3.2. The four selected lines of the Ne lamp surrounded the first resonance doublet of $\mathrm{Na}$ and the seven lines of the $\mathrm{Kr}$ lamp were spread over the range of the broad-band spectrometer.

Table 3.2: Summary of the lines that were used to assess wavelength shift for the narrow-band and broad-band systems (Kaye 1997).

\begin{tabular}{|c|c|c|}
\hline Spectrometer System & Location of Ne Lines & Location of Kr Lines \\
\hline \multirow{4}{*}{ Narrow-band } & $586.38 \mathrm{~nm}\left(17054 \mathrm{~cm}^{-1}\right)$ & \\
& $595.57 \mathrm{~nm}\left(16791 \mathrm{~cm}^{-1}\right)$ & - \\
& $608.62 \mathrm{~nm}\left(16431 \mathrm{~cm}^{-1}\right)$ & \\
\hline \multirow{4}{*}{ Broad-band } & $615.63 \mathrm{~nm}\left(16244 \mathrm{~cm}^{-1}\right)$ & \\
& & $560.47 \mathrm{~nm}\left(15373 \mathrm{~cm}^{-1}\right)$ \\
& & $614.15 \mathrm{~nm}\left(16283 \mathrm{~cm}^{-1}\right)$ \\
& & $626.55 \mathrm{~nm}\left(15960 \mathrm{~cm}^{-1}\right)$ \\
& & $640.30 \mathrm{~nm}\left(15618 \mathrm{~cm}^{-1}\right)$ \\
& & $667.57 \mathrm{~nm}\left(14980 \mathrm{~cm}^{-1}\right)$ \\
& & $692.82 \mathrm{~nm}\left(14434 \mathrm{~cm}^{-1}\right)$ \\
& & $724.25 \mathrm{~nm}\left(13087 \mathrm{~cm}^{-1}\right)$ \\
\hline
\end{tabular}

The spectral lines in Table 3.2 were identified in the background-corrected spectrometer readings of the lamps and their profiles were fit by a Gaussian function whose location was optimized by least squares regression. Gaussian profiles that had an $r^{2}<0.9$ were discarded. The line locations in Table 3.2 were subtracted from their fitted locations, producing wavelength shift values. The minimum and maximum wavelength shifts considering the spectrometer readings from all sets are summarized for the narrow-band and broad-band systems in Table 3.3.

Table 3.3: A summary of the measured maximum extents of wavelength and wavenumber shifts for the narrow-band and broad-band systems using the $0.05 \mathrm{~mm}$ and $0.01 \mathrm{~mm}$ wide slits, respectively.

\begin{tabular}{|l|c|c|}
\cline { 2 - 3 } \multicolumn{1}{c|}{} & \multicolumn{2}{c|}{ Maximum Wavelength Shift Intervals } \\
\hline Spectrometer System & $\begin{array}{c}\text { Near the Na First } \\
\text { Resonance Doublet }\end{array}$ & $\begin{array}{c}\text { Near the K First } \\
\text { Resonance Doublet }\end{array}$ \\
\hline Narrow-band & $\begin{array}{c}0.5 \text { to } 1.3 \mathrm{~nm} \\
\left(-37.6 \text { to }-14.4 \mathrm{~cm}^{-1}\right)\end{array}$ & - \\
\hline Broad-band & $\begin{array}{c}-1.1 \text { to } 0.6 \mathrm{~nm} \\
\left(-17.3 \text { to } 31.8 \mathrm{~cm}^{-1}\right)\end{array}$ & $\begin{array}{c}-0.7 \text { to } 0.6 \mathrm{~nm} \\
\left(-10.1 \text { to } 11.9 \mathrm{~cm}^{-1}\right)\end{array}$ \\
\hline
\end{tabular}


The range of shifts in Table 3.3 is small relative to the manufacturer predicted optical resolution of $0.6 \mathrm{~nm}$ and $1 \mathrm{~nm}$ for the narrow-band and broad-band systems. Thus, offaxis illumination of the spectrometer's optical bench during field measurements should not affect or impede identification of the $\mathrm{Na}$ or $\mathrm{K}$ first resonance doublet in flare spectra, or the calculation of flame temperature from soot spectra. Importantly, there are no known persistent lines that could be mistaken for a measured $\mathrm{Na}$ or $\mathrm{K}$ first resonance doublet within (or near) the shift intervals presented in Table 3.3.

\subsection{Field Measurement Protocol}

During a flare measurement in the field, the spectrometer systems were used sequentially, but the measurement procedure was otherwise the same for each system. Flare measurements were performed at UOG facilities with and without site access. Regardless of site access, a measurement location was chosen such that the flare flame was measured against a low-intensity background, where possible with the spectrometer positioned looking away from the sun. According to the Stephan-Boltzmann law, the radiative power emitted by a blackbody is proportional to its thermodynamic temperature raised to the fourth power. Thus, backgrounds of either sky or trees/bush/rolling hills (which had effective temperatures of $\$ 300 \mathrm{~K}$ ) were generally acceptable. However, when clouds were present (which could be a strong source of reflected sunlight), or when it was not possible to position the spectrometer looking away from the sun, a background of trees/bush/rolling hills was generally preferred. When site access was granted, all equipment necessary for the measurement was carried to the measurement location in a Pelican case (Model 1650) and subsequently assembled as shown in Figure 3.10.

When site access was not granted, it was more convenient to setup the spectrometer system and Toughbook laptop in the backseat of a vehicle where data could be collected through an open window. The operator conducted measurements from the backseat and often remained in the backseat during transit. Upon arriving at an UOG site with a flare to be measured, the operator and driver worked together to select a suitable measurement location as noted above. The ability to acquire data from within the vehicle drastically reduced overall measurement time and increased the number of flares that could be measured. 


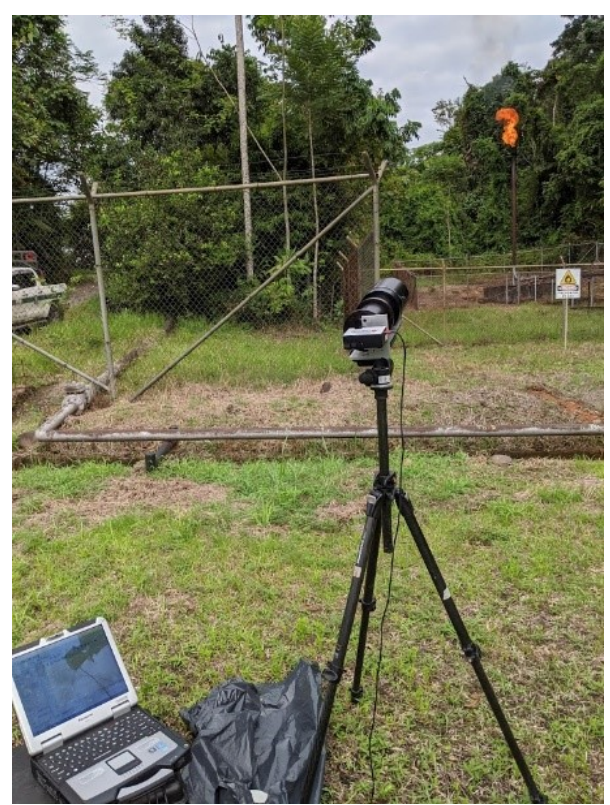

Figure 3.10: The spectrometer system and Toughbook laptop setup at a measurement location where site access was granted.

At each site, the spectrometer system was snapped into the articulating knuckle mounted on the tripod (if not already assembled) and crudely pointed towards the flare. The spectrometer was powered on and communication between the spectrometer and Toughbook laptop was established via the Oceanview program. In the program: the integration time was set to $1 \mathrm{msec}$, a file directory for the particular flare was created, the continuous save feature was engaged, and the measurement duration was typically set between 3 to $6 \mathrm{~min}$.

The program previewed spectra in real time $(5 \mathrm{msec}$ refresh rate at an integration time of $1 \mathrm{msec}$ ) when not saving data to memory, which allowed the flare flame to be scanned for possible emission signatures prior to beginning a measurement. If a suspected emission signature was found, its intensity was maximized by finely adjusting the aim of the spectrometer system. When no signature was found, the flame was still measured and the aim was instead adjusted to maximize the intensity of soot emission. The measurement was initiated once the aim was optimized.

If upon completion of a measurement the operator was not certain that the acquired flare spectra contained evidence of a suspected emission signature, additional measurements were performed. These additional measurements involved incrementally increasing the 
integration time, re-scanning the flame, finely adjusting the aim, and reinitiating the measurement. Additional measurements continued until the emission from the flare flame saturated the linear detector or a suspected atomic emission signature was observed. A measurement was repeated at a particular integration time if the flare flame was subjected to strong/gusting crosswind or a suspected atomic emission signature appeared sporadically in the previewed spectra.

Following the last measurement, background intensity levels (dark count) were acquired at each relevant integration time. This involved installing the lens cap and covering the entire apparatus with two contractor garbage bags to reduce the chance of stray light reaching the linear detector. The duration of each background noise measurement was typically $30 \mathrm{~s}$.

\subsection{Experimental Estimation of the Detectability of the Na and K First Resonance Doublets}

Turbulent, open atmosphere flare flames have a continually changing structure (i.e., thickness, thermal and concentration gradients, and orientation or solid angle as viewed from the spectrometer system) and experience variable energy loss (in the form of heat and radiation) to the surroundings. These conditions inhibit quantitative measurement of the number density of $\mathrm{Na}$ and $\mathrm{K}$ atoms in the flame along the line of site. Nevertheless, a rough preliminary estimate of the detection sensitivity of the spectrometer systems was attempted via controlled laboratory experiments. This preliminary detection sensitivity was considered to be the minimum salt-loading required to produce detectable emission signatures of $\mathrm{Na}$ or $\mathrm{K}$ in a lab-scale flare flame under optimal measurement conditions (i.e., close distance, no background, relative stable flare in regards to its fuel flow rate and orientation or sway).

The relative intensity of a measured emission line may be sensitive to, in part, the optical thickness / brightness of the flame (considering atomic and soot emission), the presence of more readily ionized compounds (e.g., dissociation of $\mathrm{KCl}$ may hinder that of $\mathrm{NaCl}$ ), the measurement distance (e.g., reduction in intensity due to the inverse-squared distance), and the aim of the spectrometer system (i.e., species are not uniformly distributed throughout flame). This study performed exploratory experiments to determine the weakest salt 
loading rate of $\mathrm{NaCl}$ and $\mathrm{KCl}$ that produced detectable atomic emission lines of $\mathrm{Na}$ and $\mathrm{K}$ in a lab-scale fare flame, when measured using the narrow-band and broad-band spectrometers using $0.05 \mathrm{~mm}$ wide and $0.01 \mathrm{~mm}$ wide slits, respectively.

The lab-scale flare flame was generated at the Carleton University Flare Facility (CUFF) burning a hydrocarbon fuel mixture typical of the Alberta UOG industry, shown in Table 3.4 .

Table 3.4: Hydrocarbon fuel mixture typical of the Alberta UOG industry used during test to estimate the detectability of the $\mathrm{Na}$ and $\mathrm{K}$ first resonance doublet.

\begin{tabular}{|l|c|}
\hline Species & Volume Fraction [\%] \\
\hline $\mathrm{CH}_{4}$ (Methane) & 75.68 \\
\hline $\mathrm{C}_{2} \mathrm{H}_{6}$ (Ethane) & 11.52 \\
\hline $\mathrm{C}_{3} \mathrm{H}_{8}$ (Propane) & 6.06 \\
\hline $\mathrm{C}_{4} \mathrm{H}_{10}$ (Butane) & 2.60 \\
\hline $\mathrm{C}_{5} \mathrm{H}_{12}$ (Pentane) & 0.78 \\
\hline $\mathrm{C}_{6} \mathrm{H}_{14}$ (Hexane) & 0.23 \\
\hline $\mathrm{C}_{7} \mathrm{H}_{16}$ (Heptane) & 0.21 \\
\hline $\mathrm{N}_{2}$ (Nitrogen gas) & 1.70 \\
\hline $\mathrm{CO}_{2}$ (Carbon dioxide) & 1.22 \\
\hline
\end{tabular}

A customised 2 in diameter burner system equipped with an aerosol injection system was used similar to the experiments performed by Jefferson (2017). An atomizer (Sono-Tek model \#: NZL120CO) mounted inside the burner injected a stream of salt laden aerosols directly into the combustion zone of the flame. The median droplet diameter was approximately $0.019 \mathrm{~mm}$. The salt solutions were delivered to the atomizer via a precision micro gear pump (Ismatec/Cole-Parmer ISM901B) with a suction-shoe head (Ismatec/Cole-Parmer L21834) (Jefferson 2017). The salt solutions were made by mixing pure $\mathrm{NaCl}$ or $\mathrm{KCl}$ crystals (solute) with distilled water (solvent) in varying mass concentrations, defined as:

$$
\text { Mass Concentration }=\frac{\text { mass of solute }}{\text { mass of solute }+ \text { mass of solvent }}
$$

An initial solution with a mass concentration of $0.01 \%$ was created by weighing out the solute and solvent and mixing them together. Solutions with concentrations $<0.01 \%$ were 
produced by further diluting the initial solution with measured amounts of distilled water. Details of the flame and fuel / aerosol stream are summarized in Table 3.5.

Table 3.5: Flame and fuel / aerosol stream properties for the experiments used to estimate the detectability of $\mathrm{Na}$ and $\mathrm{K}$ as a function of salt loading rate.

\begin{tabular}{|c|c|}
\hline Parameter & Setting \\
\hline \multicolumn{2}{|c|}{ Fuel stream / Flame } \\
\hline Flowrate [SLPM at $273.15 \mathrm{~K}, 1 \mathrm{~atm}]$ & 109.5 \\
\hline Composition [-] & $\mathrm{C} 1$ to $\mathrm{C} 7, \mathrm{~N}_{2}, \mathrm{CO}_{2}$ - typical of field $\mathrm{AB}-\mathrm{H} 9$ \\
\hline Higher Heating Value [MJ kg-1] & 51 \\
\hline Adiabatic flame temperature $[\mathrm{K}]$ & 2236.5 \\
\hline $\begin{array}{l}\text { Calculated flame length [m] } \\
\text { (Delichatsios et al. 1984) }\end{array}$ & 1.5 \\
\hline \multicolumn{2}{|c|}{ Aerosol stream } \\
\hline Liquid flow rate $\left[\mathrm{ml} \mathrm{min}^{-1}\right]$ & 7.5 \\
\hline Ultrasonic generator power level [\%] & 44 \\
\hline
\end{tabular}

\subsubsection{Sensitivity Measurements}

The sensitivity of the spectrometer systems to $\mathrm{Na}$ or K emission was estimated by recording the spectrometer readings of flames as the salt loading rate (i.e., mass concentration of the $\mathrm{NaCl}$ or $\mathrm{KCl}$ solution) was reduced until the first resonance doublet of $\mathrm{Na}$ or $\mathrm{K}$ was no longer detectable in the flame spectra. Tests estimated the sensitivity of the narrow-band system to $\mathrm{Na}$ emission, and the broad-band system to K emission.

Preliminary experiments revealed that the intensity of the first resonance doublets were sensitive to the aim of the spectrometer system. Therefore, to conduct a meaningful sensitivity experiment, the spectrometer aim was finely adjusted during preliminary tests and locked in place for the duration of the sensitivity experiments. This aim maximized the intensity of first resonance doublets at the setup location of the spectrometer system. The spectrometer system aimed at a lab-scale flare flame that was generated using the custom 2 in burner with the mesh screens (which stabilize the flame) is shown in Figure 3.11 . 


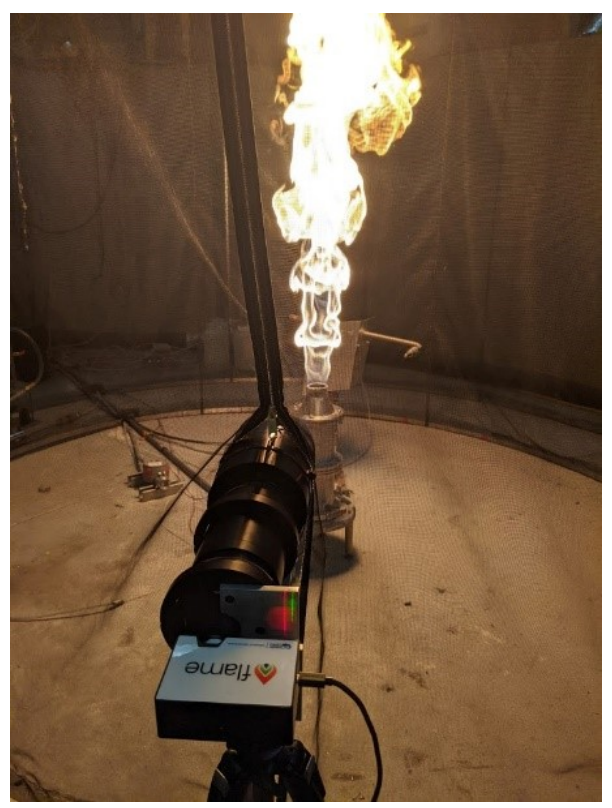

Figure 3.11: The spectrometer system aimed at the lab-scale flare flame generated using the custom 2 in burner at the CUFF. The reflective igniter seen in the background was removed prior to performing the assessment.

Unfortunately, within the confines of the lab it was not possible to replicate field conditions, especially the stand-off distance between the spectrometer and the flame. For the preliminary tests presented here, the spectrometer system was positioned at an arbitrary distance away from the burner, $\sim 2 \mathrm{~m}$, and pointed at a slight inclination toward the centerline of the flame, at $\sim 1 / 3$ of the flame's height. These preliminary experiments are expected to overestimate the sensitivity of the spectrometer system due to optimal flame and measurement conditions. This is mostly because the short stand-off distance of the lab measurements allows simpler alignment and near-perfect filling of the spectrometer. Moreover, the volume of the lab-scale flare flame that is observed by the spectrometer (at a distance of $\sim 2 \mathrm{~m}$ ) is assumed to be as uniform as a flare flame could be (in terms of flame thickness and the concentrations of emitting species), and to be the densest region of atomic species within the flame.

The sensitivities calculated under lab conditions are likely not the same as flare flames measured at a stand-off distance of $125 \mathrm{~m}$, even if the observed volume of flame is similar. During a field measurement there is a lesser chance the FOV is completely and consistently filled and that the flame is uniform over the FOV. Furthermore, the fuel and aerosol streams may be unsteady under field conditions, and the effect of strong cross-winds 
(steady and gusting) could dilute species concentrations. These issues contribute to a lesser sensitivity to similar salt loading rates for flare flames under field conditions because the density of their outgoing atomic emission field will almost certainly be less than that of a lab-scale flare flame. Ultimately, without an absolute calibration, the intensity of atomic emission lines measured in the field cannot be compared to those from the lab without knowing fuel or aerosol stream characteristics, or the effect that stand-off distance has on signal intensity.

The preliminary sensitivity measurements involved recording two identical $45 \mathrm{~s}$ duration spectrometer readings, the first while distilled water aerosols were injected and the second while salt solution aerosols were injected. The spectrometer readings were recorded only after the lab-scale flare flame and the liquid injection were given $\sim 2$ min to reach an (assumed) steady state. Following the second spectrometer reading the liquid delivery system (i.e., the liquids reservoir, the pump, and the atomizer) were flushed with distilled water.

For each sensitivity measurement, spectrometer readings for the distilled water and salt solution injection were transformed into the wavenumber domain (see Chapter 4 for a complete breakdown of this process), and averaged to produce a mean spectrometer reading for each injection type. The average spectrometer reading for distilled water injection was subtracted from the salt solution injection to isolate first resonance doublet of $\mathrm{Na}$ or $\mathrm{K}$ from its background. The isolated first resonance doublets of $\mathrm{Na}$ and $\mathrm{K}$ are shown in Figure 3.12 for the two weakest salt loading rates in which atomic emission was still present. 


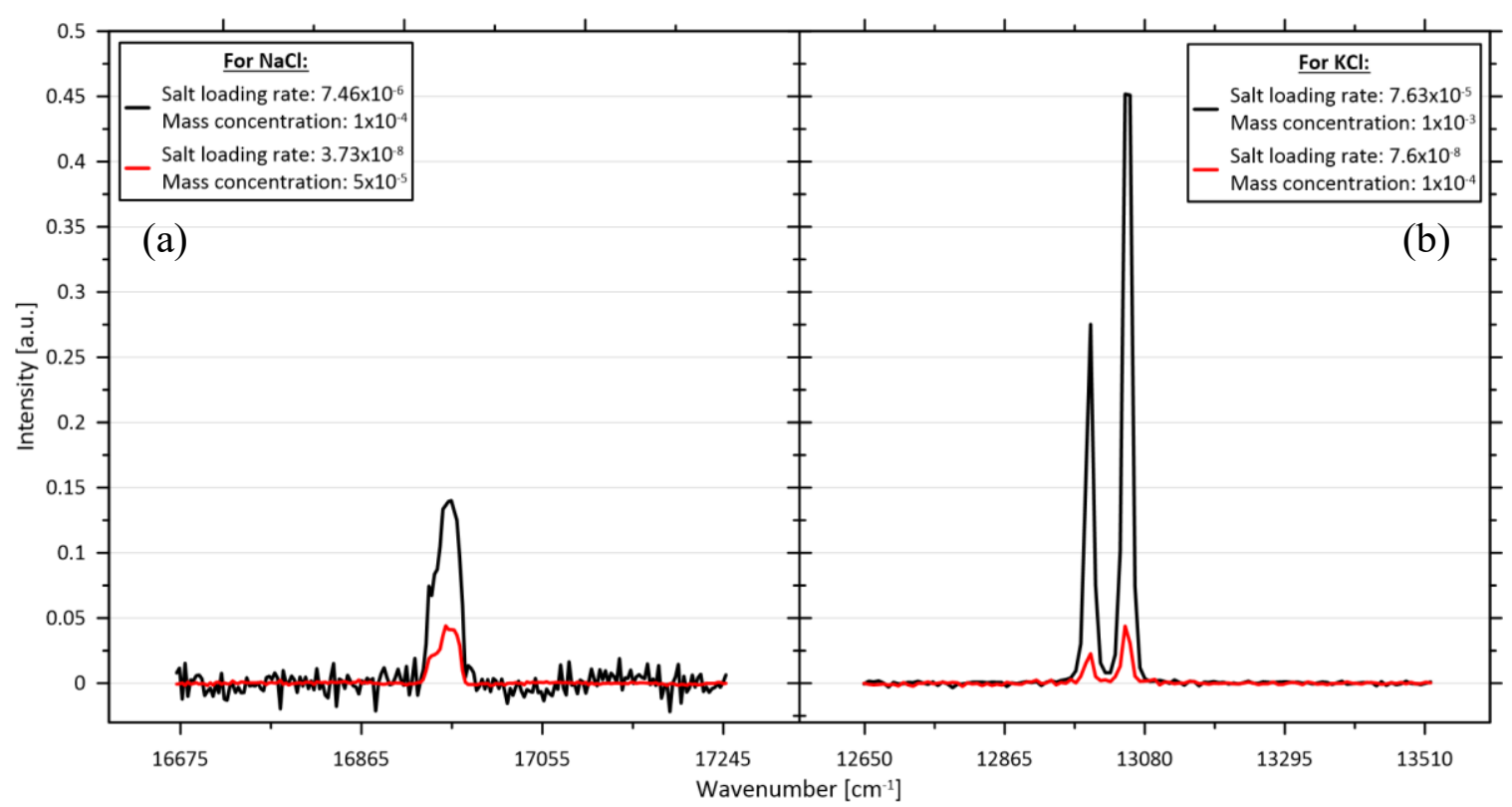

Figure 3.12: Average sensitivity measurement spectrum that produced the sensitivity limit for the (a) narrow-band system and (b) broad-band systems when measuring $\mathrm{Na}$ and $\mathrm{K}$ emission, respectively.

The sensitivity limit of the broad-band system was for the $0.01 \mathrm{~mm}$ wide slit.

The spectra presented in Figure 3.12 suggest that the weakest salt loading rate that produces detectible emission of $\mathrm{Na}$ is $\left.3.73 \times 10^{-8}\left[(\mathrm{~kg} \text { of salt }) \mathrm{sec}^{-1}\left((\mathrm{~kg} \text { of fuel }) \mathrm{sec}^{-1}\right)\right)^{-1}\right]$ as measured by the narrow-band system at a distance of $2 \mathrm{~m}$. Similarly, the lowest salt loading that produces a detectable emission of $\mathrm{K}$ is $\left.7.6 \times 10^{-8}\left[(\mathrm{~kg} \text { of salt }) \mathrm{sec}^{-1}\left((\mathrm{~kg} \text { of fuel }) \mathrm{sec}^{-1}\right)\right)^{-1}\right]$ as measured by the broad-band system at a distance of $2 \mathrm{~m}$. These results are summarized in Table 3.6.

Table 3.6: Summary of experiments to estimate the detection sensitivities of salt emission at a distance of $2 \mathrm{~m}$. The sensitivity limit of the broad-band system was estimated using the $0.01 \mathrm{~mm}$ wide slit.

\begin{tabular}{|l|l|l|}
\hline Sensitivity Test Parameter & Narrow-band & Broad-band \\
\hline Slit width $[\mathrm{mm}]$ & 0.05 & 0.01 \\
\hline Species & $\mathrm{Na}$ & $\mathrm{K}$ \\
\hline Solute & $\mathrm{NaCl}$ & $\mathrm{KCl}$ \\
\hline Mass concentration of solution [\%] & $5 \times 10^{-5}$ & $1 \times 10^{-4}$ \\
\hline Mass flow rate of solution to flame $\left[\mathrm{kg} \mathrm{sec}^{-1}\right]$ & $1.32 \times 10^{-4}$ & $1.35 \times 10^{-4}$ \\
\hline Mass flow rate of salt to flame $\left[\mathrm{kg} \mathrm{sec}^{-1}\right]$ & $6.61 \times 10^{-11}$ & $1.35 \times 10^{-10}$ \\
\hline Mass flow rate of fuel to flame $\left[\mathrm{kg} \mathrm{sec}^{-1}\right]$ & $1.77 \times 10^{-3}$ & $1.77 \times 10^{-3}$ \\
\hline Salt loading [(kg of salt) sec $\left.\left.{ }^{-1}\left(\mathrm{~kg} \mathrm{of} \mathrm{fuel)} \mathrm{sec}^{-1}\right)\right)^{-1}\right]$ & $3.73 \times 10^{-8}$ & $7.63 \times 10^{-8}$ \\
\hline Estimated volume fraction of salt $[-]$ & $1.07 \times 10^{-9}$ & $1.69 \times 10^{-9}$ \\
\hline
\end{tabular}

a (considering only reactants - fuel, stoichiometric air, water, and salt) 


\section{Chapter 4 Data Processing}

\subsection{Initial Processing}

Initial processing of spectrometer data acquired in the field included subtracting an average noise spectrum from the flare spectra, cropping the flare spectra to a wavelength range of interest, applying the relative spectral response calibration curves to correct the shape of the spectra, and transforming the flare spectra from the wavelength domain to the wavenumber domain. Each of these steps is detailed by example below. Processing was identical for each spectrometer, other than the differences in wavelength range.

\subsubsection{Subtracting the Noise from, and Cropping the Raw Flare Spectra}

As detailed in Chapter 3, a typical flare measurement involved obtaining flare spectra and noise spectra at various integration times. For each integration time, average noise spectra were calculated and then subtracted from the measured flare spectra. The resulting spectra were cropped to the ranges of interest summarized in Table 4.1.

Table 4.1: Intervals used to crop raw flame spectra.

\begin{tabular}{|l|c|}
\hline Spectrometer & Cropped Range of Flame Spectra \\
\hline Narrow-band & $\begin{array}{c}484 \text { to } 606 \mathrm{~nm} \\
\left.\text { (16502 to } 20661 \mathrm{~cm}^{-1}\right)\end{array}$ \\
\hline Broad-band & $\begin{array}{r}500 \text { to } 900 \mathrm{~nm} \\
\left(11111 \text { to } 20000 \mathrm{~cm}^{-1}\right)\end{array}$ \\
\hline
\end{tabular}

Figure 4.1 shows examples of a measured flare spectrum, the average noise spectrum, and the noise-subtracted spectrum (all of which have been cropped) for the narrow- and broadband systems. Notice the strong atomic emission signatures at $\sim 590 \mathrm{~nm}$ in the narrow and broad spectra, and at $\sim 767 \mathrm{~nm}$ in the broad spectrum. In Section 4.3, these atomic emission signatures are fit by the atomic emission model derived in Chapter 2 to prove that they match the first resonance doublet of $\mathrm{Na}$ and $\mathrm{K}$, respectively. 


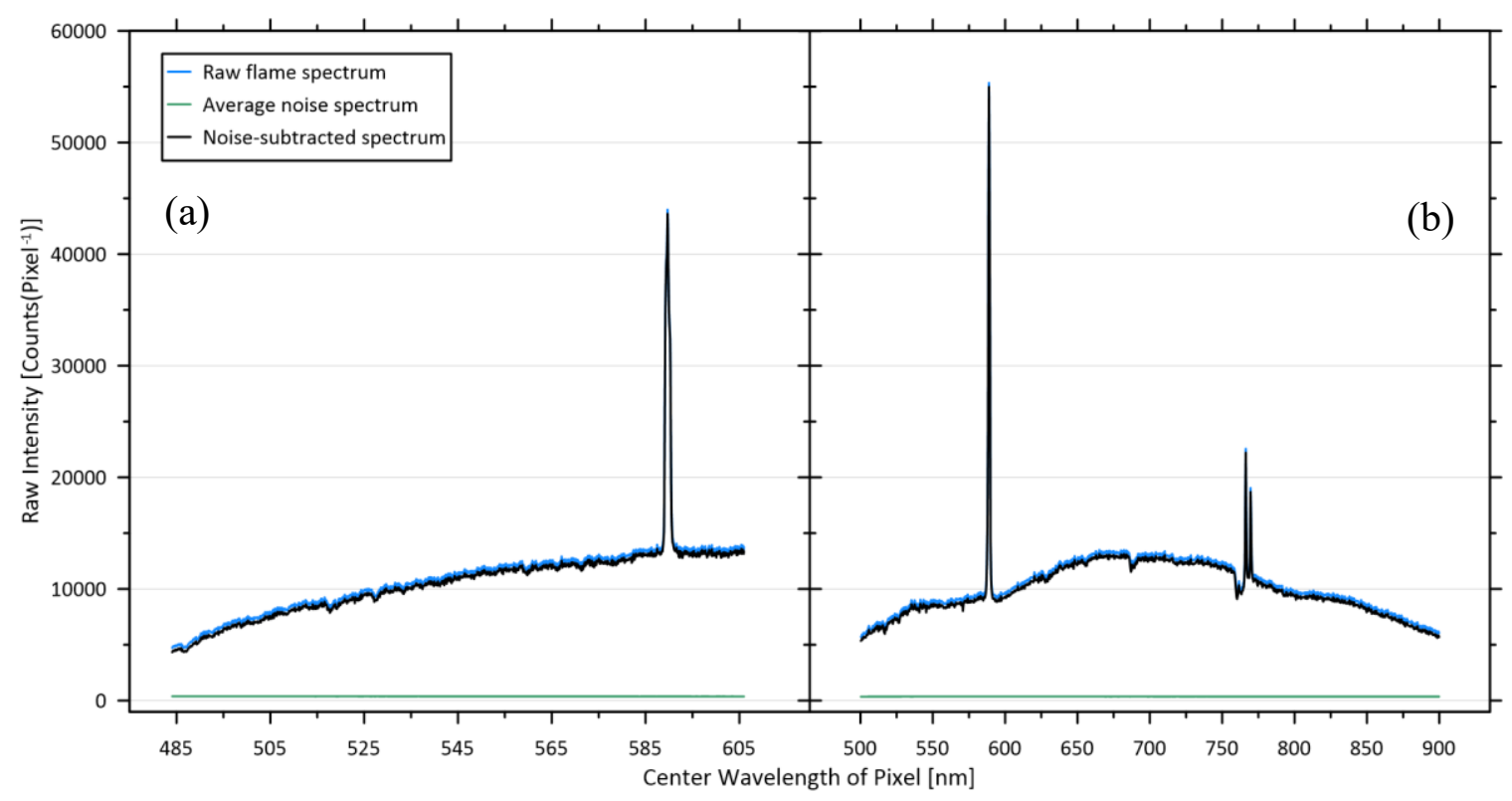

Figure 4.1: Example measured flare spectra, average noise spectra, and noise-subtracted spectra for the (a) narrow-band system and (b) broad-band system (with a $0.01 \mathrm{~mm}$ slit).

\subsubsection{Applying the Relative Spectral Response Calibration Curves}

The soot temperature, atomic emission, and molecular absorption models presented in Chapter 2 require noise-subtracted spectra with an accurate spectral shape. This was achieved by calibrating the noise subtracted spectra with the relative spectral response curves described in Chapter 3. Fundamentally, this calibration transforms the raw units of noise-subtracted spectra [counts pixel ${ }^{-1}$ ] into units that are proportional to spectral radiance $\left[\mathrm{W} \mathrm{m} \mathrm{m}^{-2} \mathrm{sr}^{-1}\left(\mathrm{~nm}^{-1}\right)^{-1}\right]$.

A unique relative spectral response curve was calculated for each noise-subtracted spectrum. For each point along the spectrum, an intensity-specific calibration factor was determined by linearly interpolating among the three relative spectral response curves for different intensities found in Chapter 3. The resulting calibration curve (comprised of linearly interpolated calibration factors) was then multiplied by the noise subtracted spectra to generate a "calibrated" spectrum. Starting from the same noise-subtracted spectra plotted in Figure 4.1, Figure 4.2 shows the associated unique relative spectral response curves and the resulting calibrated spectra for the narrow-band and broad-band systems. 


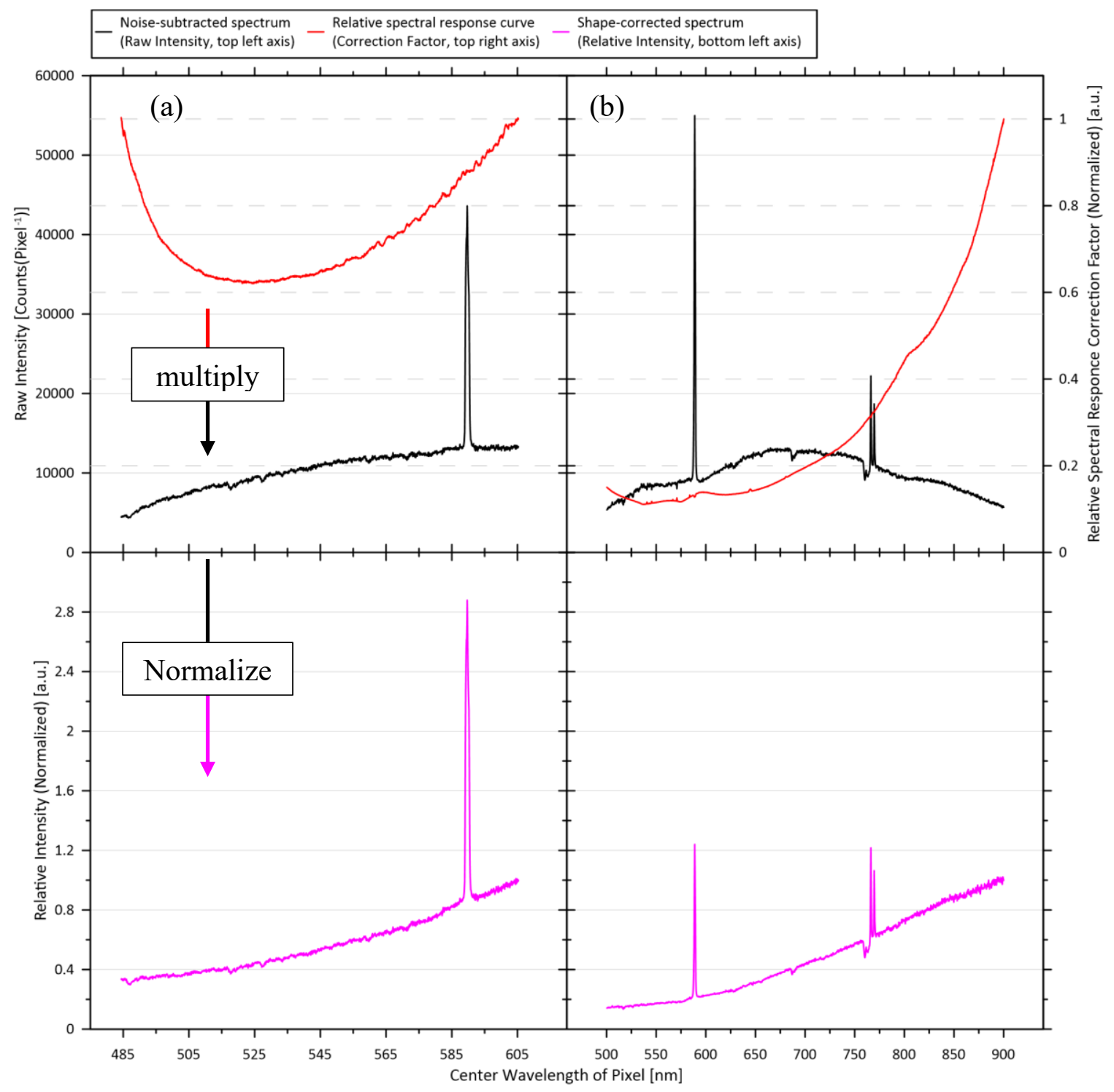

Figure 4.2: The noise subtracted spectra from Figure 4.1, their unique calibration curves and the final calibrated spectra for the (a) narrow-band system and (b) broad-band system with a $0.01 \mathrm{~mm}$ slit.

The relative spectral response curves and calibrated spectra shown in Figure 4.2 were normalized by the intensity and calibration factor at the wavelength indicated in Table 4.2. Normalization was performed to facilitate direct visual comparison of their shapes and to keep working values consistent.

Table 4.2: Normalization wavelengths for calibrated spectra.

\begin{tabular}{|l|c|}
\hline Spectrometer & Normalization Wavelength \\
\hline Narrow-band & $606 \mathrm{~nm}\left(16502 \mathrm{~cm}^{-1}\right)$ \\
\hline Broad-band & $900 \mathrm{~nm}\left(11111 \mathrm{~cm}^{-1}\right)$ \\
\hline
\end{tabular}




\subsubsection{Transformation from the Wavelength to Wavenumber Domain}

The soot temperature, atomic emission, and molecular absorption models required calibrated spectra to be transformed from the wavelength domain to the wavenumber domain. The transformation is necessary because these models were developed for the wavenumber domain, consistent with the sources that were referenced during the derivation (Goldenstein et al. 2017; Hanson et al. 2016; Alkemade et al. 1982). However, because the spectrometer systems output data at constant wavelength increments, transforming the calibrated spectra straight to the wavenumber domain results in wavenumber spectra that have unevenly spaced data points. This complicates the fitting of the soot temperature models via least squares regression since a direct wavelength to wavenumber transformation results in the lower wavenumber region having more data points and thus more weight during least squares regression than the higher wavenumber region.

Instead, the calibrated spectra in the wavelength domain were transformed to the wavenumber domain by identifying the wavenumber range that was measured by the spectrometer system and re-sampling at evenly spaced wavenumbers within this range. The number of re-sampled wavenumbers was chosen to match the number of data points in the original calibrated wavelength spectra as shown in Table 4.3.

Table 4.3: Number of data points contained within the calibrated spectra and normalization wavenumbers for the resampled data in the wavenumber domain.

\begin{tabular}{|l|c|c|}
\hline Spectrometer & Amount of Data Points Remaining & Normalization Wavenumber \\
\hline Narrow-band & 1401 & $\begin{array}{c}19000 \mathrm{~cm}^{-1} \\
(526 \mathrm{~nm})\end{array}$ \\
\hline Broad-band & 1173 & $\begin{array}{c}14900 \mathrm{~cm}^{-1} \\
(671 \mathrm{~nm})\end{array}$ \\
\hline
\end{tabular}

The intensity at each re-sampled wavenumber was found via linear interpolation. The conversion involved multiplying the intensity of each data point by the wavelength range that it measured, and dividing it by the equivalent wavenumber range (See diagram in Figure 4.3 for details). The re-sampled wavenumber spectra were normalized by the intensity at the wavenumber indicated in Table 4.3 prior to being fit by soot temperature models derived in Chapter 2. The calibrated wavelength spectrum from Figure 4.2 and its 
re-sampled wavenumber spectrum are shown in Figure 4.3 for the narrow-band and broadband systems.
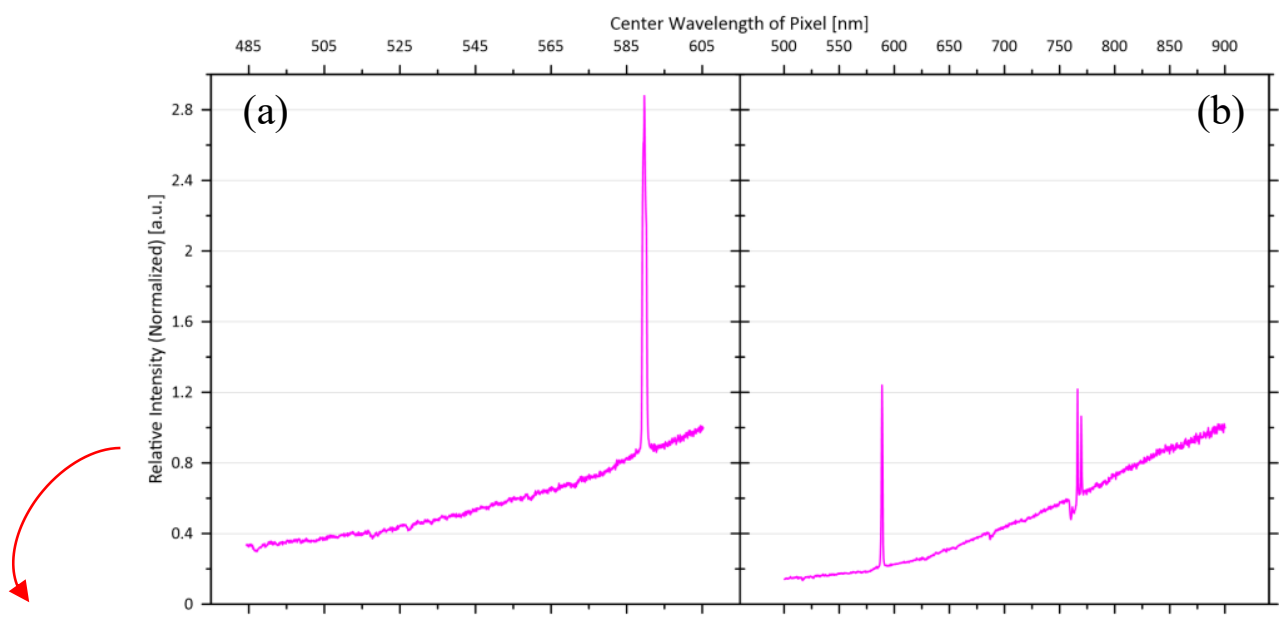

Wavelength to wavenumber conversion e.g.: 5 counts $\left(\mathrm{nm}^{-1}\right) @$ center of pixel $(555 \mathrm{~nm})$ Pixel measured $2 \mathrm{~nm}$ in wavelength domain
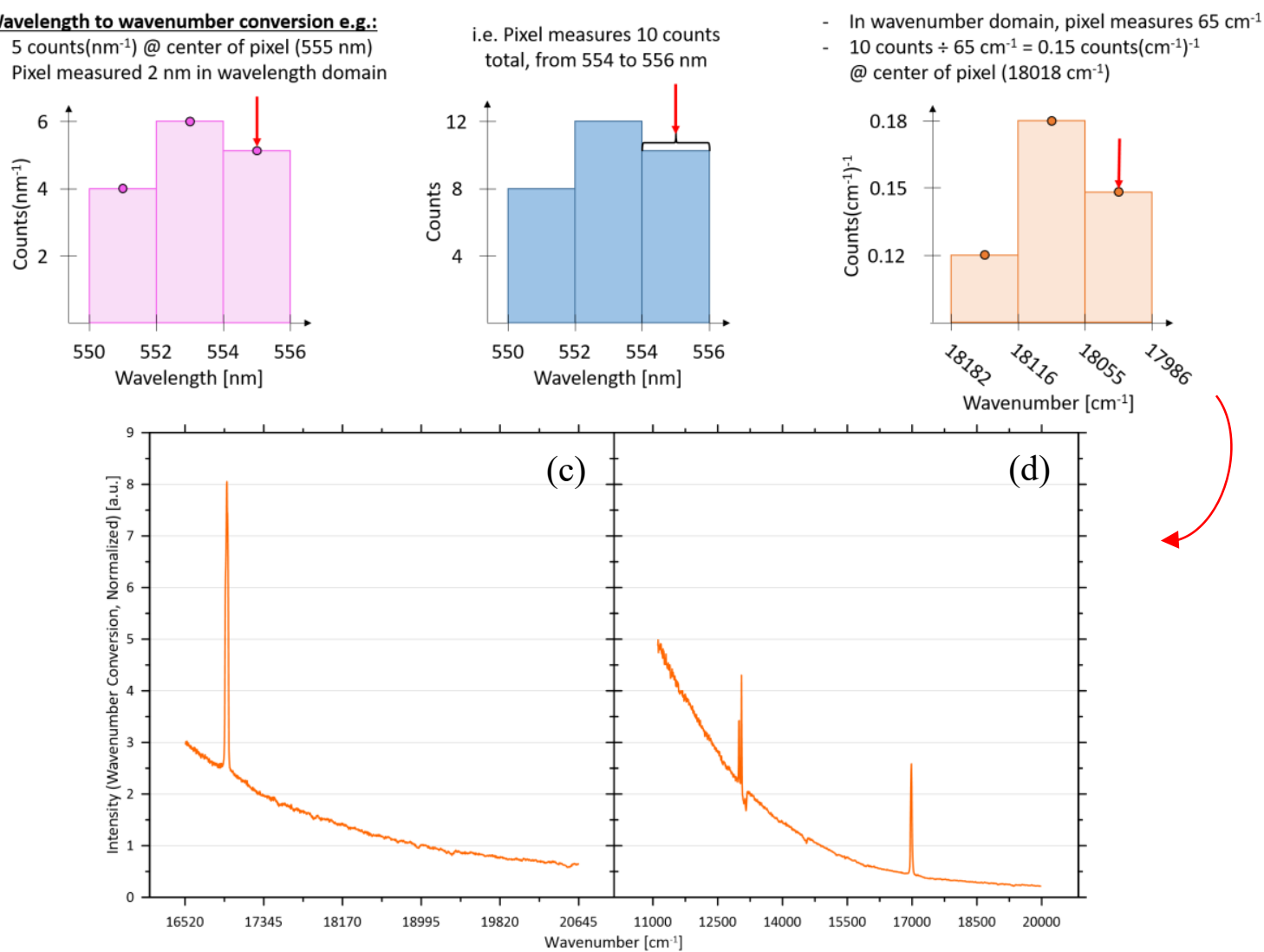

Figure 4.3: The calibrated spectra from Figure $4.2(\mathrm{a} \& \mathrm{~b})$ and their re-sampled wavenumber spectrum for the (c) narrow-band system and (d) broad-band system with a $0.05 \mathrm{~mm}$ and $0.01 \mathrm{~mm}$ wide slit, respectively. 


\subsection{Determining the Effective Temperature of a Flare Flame}

The effective temperature $\left(T_{g}\right)$ of a flare measurement is important to this study for two reasons: $T_{g}$ is considered to be the electronic temperature of sodium $(\mathrm{Na})$ and potassium (K) (which is otherwise an unknown variable in the atomic emission model), and the inferred $T_{g}$ from fitting broad-band soot emission can be used to filter excessively noisy spectra or spectra that do not follow the expected blackbody trend as can occur when the turbulent flare flame moves in and out of the field of view during field measurements.

The effective flame temperature was calculated as the weighted average of the optically thin (OTN) and optically thick (OTK) models as described in Chapter 2 (see Eq. (2.8)). These soot temperature models were fit to measured spectra with the wavenumber intervals in Table 4.4 removed, as they correspond to spectral features produced by radiative processes such as the chemiluminescence of radicals, the atomic emission of species within the flare flame, and the molecular absorption of species in ambient air. In general, the excluded wavenumber intervals were slightly narrower than the suggested ranges of spectral features found in literature, since at the present conditions and species concentrations, the low intensity "wings" of these spectral features near the edges of the ranges had no observable effect in the measured spectra.

As indicated in Table 4.4, the broadband $\mathrm{CO}_{2}{ }^{*}$ emission due to chemiluminescence was not removed from wavenumber spectra that were measured by the narrow-band systems. The gradual trend of its continuous emission did not have an observable effect on the identification of the $\mathrm{Na}$ first resonance doublet in narrow-band wavenumber spectra. The soot temperature models were fit to both narrow-band and broad-band soot spectra but due to its extended wavenumber range, the broad-band soot temperatures were used to determine $T_{g}$. Soot temperatures from the narrow-band data were still used to filter noisy spectra or spectra that did not follow the expect trend of soot emission. 
Table 4.4: Summary of the source and wavenumber range of spectra features that were removed from the wavenumber spectra to isolate soot emission.

\begin{tabular}{|c|c|c|c|c|}
\hline Species $^{a}$ & $\begin{array}{l}\text { Radiative } \\
\text { Process }^{b}\end{array}$ & Description in Literature & $\begin{array}{l}\text { Wavenumbers } \\
\text { Removed From } \\
\text { Spectra }\end{array}$ & $\begin{array}{l}N / B / \\
N+B^{c}\end{array}$ \\
\hline $\mathrm{CO}_{2}{ }^{*}$ & $\mathrm{CL}$ & $\begin{array}{l}\text { Broadband emission } \\
15385-29412 \mathrm{~cm}^{-1} \\
(340-650 \mathrm{~nm}) \\
\text { Maguire-Boyle and Barron (2014) }\end{array}$ & $\begin{array}{l}>16667 \mathrm{~cm}^{-1} \\
(<600 \mathrm{~nm})\end{array}$ & B \\
\hline $\mathrm{C}_{2}{ }^{*}$ & $\mathrm{CL}$ & $\begin{array}{l}\text { Swan band }(\Delta v=-1) \\
17740-18172 \mathrm{~cm}^{-1} \\
(550.3-563.7 \mathrm{~nm}) \\
\text { Mekhrengin et al. }(2020)\end{array}$ & $\begin{array}{l}17857-18182 \mathrm{~cm}^{-1} \\
(550-560) \mathrm{nm}\end{array}$ & $N+B$ \\
\hline $\mathrm{Na}$ & $\mathrm{AE}$ & $\begin{array}{l}\text { First resonance doublet } \\
16961,16978 \mathrm{~cm}^{-1} \\
(589,589.59 \mathrm{~nm}) \\
\text { Kramida et al. (2020) }\end{array}$ & $\begin{array}{l}16835-17050 \mathrm{~cm}^{-1} \\
(586.5-594 \mathrm{~nm})\end{array}$ & $N+B$ \\
\hline $\mathrm{O}_{2}$ & MA & $\begin{array}{l}\text { B band } \\
14388-14599 \mathrm{~cm}^{-1} \\
(685-695 \mathrm{~nm}) \\
\text { Gordon et al. (2017) and Ding et al. } \\
(2016)\end{array}$ & $\begin{array}{l}14409-14599 \mathrm{~cm}^{-1} \\
(685-694 \mathrm{~nm})\end{array}$ & B \\
\hline $\mathrm{H}_{2} \mathrm{O}$ & MA & $\begin{array}{l}\text { VIS band }^{d} \\
13400-14500 \mathrm{~cm}^{-1} \\
(690-746 \mathrm{~nm}) \\
\text { Gordon et al. (2017) }\end{array}$ & $\begin{array}{l}13624-14006 \mathrm{~cm}^{-1} \\
(714-734 \mathrm{~nm})\end{array}$ & B \\
\hline $\mathrm{O}_{2}$ & MA & $\begin{array}{l}\text { A band } \\
12903-13245 \mathrm{~cm}^{-1} \\
(755-775 \mathrm{~nm}) \\
\text { Gordon et al. (2017) and Ding et al. } \\
(2016)\end{array}$ & $\begin{array}{l}12887-13333 \mathrm{~cm}^{-1} \\
(750-776 \mathrm{~nm})\end{array}$ & B \\
\hline K & $\mathrm{AE}$ & $\begin{array}{l}\text { First resonance doublet } \\
12989,13046 \mathrm{~cm}^{-1} \\
(766.49,769.9 \mathrm{~nm}) \\
\text { Kramida et al. }(2020)\end{array}$ & $\begin{array}{l}12937-13115 \mathrm{~cm}^{-1} \\
(762.5-773 \mathrm{~nm})\end{array}$ & B \\
\hline $\mathrm{H}_{2} \mathrm{O}$ & MA & $\begin{array}{l}\text { VIS-NIR band } \\
11680-12720 \mathrm{~cm}^{-1} \\
(786-856 \mathrm{~nm}) \\
\text { Gordon et al. (2017) }\end{array}$ & $\begin{array}{l}11905-12376 \mathrm{~cm}^{-1} \\
(808-840 \mathrm{~nm})\end{array}$ & B \\
\hline $\mathrm{H}_{2} \mathrm{O}$ & MA & $\begin{array}{l}\text { NIR band } \\
10150-11200 \mathrm{~cm}^{-1} \\
(893-985 \mathrm{~nm}) \\
\text { Gordon et al. (2017) }\end{array}$ & $\begin{array}{l}<11300 \mathrm{~cm}^{-1} \\
(>885 \mathrm{~nm})\end{array}$ & B \\
\hline
\end{tabular}

${ }^{\text {a }}$ Radical is defined by $(*)$

${ }^{\mathrm{b}} \mathrm{CL}=$ chemiluminescence, $\mathrm{AE}=$ atomic emission, and $\mathrm{MA}=$ molecular absorption

${ }^{\mathrm{c}} \mathrm{N}=$ narrow-band system, $\mathrm{B}=$ broad-band system, and $\mathrm{N}+\mathrm{B}=$ narrow-band and broad-band systems

${ }^{\mathrm{e}} \mathrm{VIS}$ band $=$ band in visible spectrum

${ }^{\mathrm{e}}$ VIS-NIR band $=$ band on threshold of visible and near-infrared spectrum

${ }^{\mathrm{f}} \mathrm{NIR}$ band $=$ band in near-infrared spectrum 
Example spectra and excluded wavenumber intervals listed in Table 4.4 are shown in Figure 4.4 for the narrow-band and broad-band systems. The remaining soot spectrum (shown in orange) could then be fit by the OTN and OTK soot temperature models.

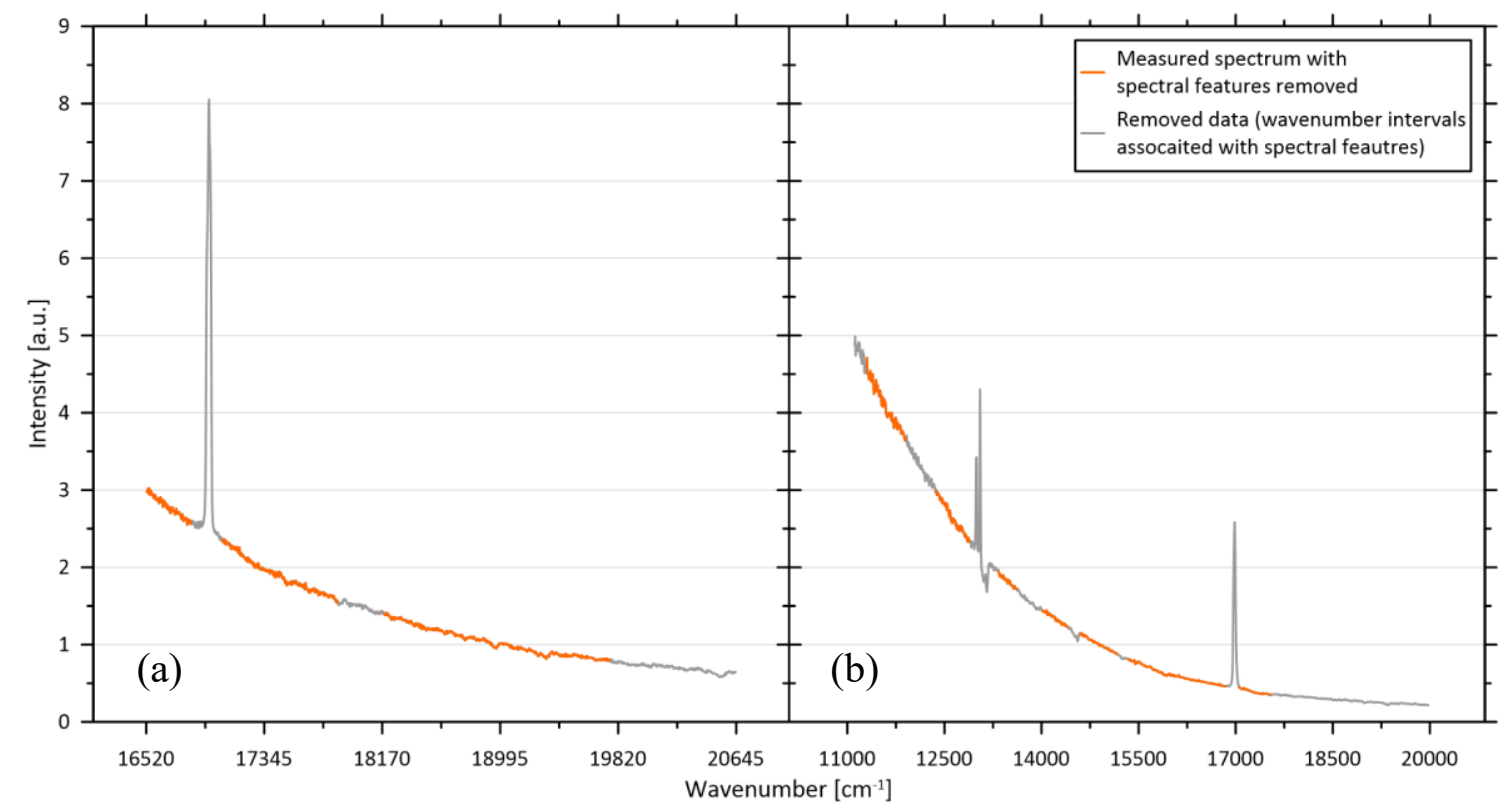

Figure 4.4: The wavenumber spectra from Figure 4.3 with excluded wavenumber intervals (grey) from Table 4.4 for (a) the narrow-band system with the $0.05 \mathrm{~mm}$ wide slit and (b) the broad-band system with the $0.01 \mathrm{~mm}$ wide slit.

\subsubsection{Fitting the Soot Temperature Models to Soot Spectra and Calculating the Effective Temperature}

The OTN and OTK soot temperature models presented in Chapter 2 were derived to fit the $\log$ transform of the normalized soot spectra via least squares regression. The intensity of soot spectra was normalized during the wavenumber transformation by the intensity at the wavenumber indicated in Table 4.3 (Section 4.1.3), and the natural logarithm of normalized intensity of soot spectra were calculated prior to fitting the soot temperature models via least squares regression. Figure 4.5 shows an example of the $\log$ transform of a normalized soot spectrum overlaid with the fitted OTN and OTK models for the narrow-band and broad-band systems with a $0.05 \mathrm{~mm}$ and $0.01 \mathrm{~mm}$ wide slit, respectively. 


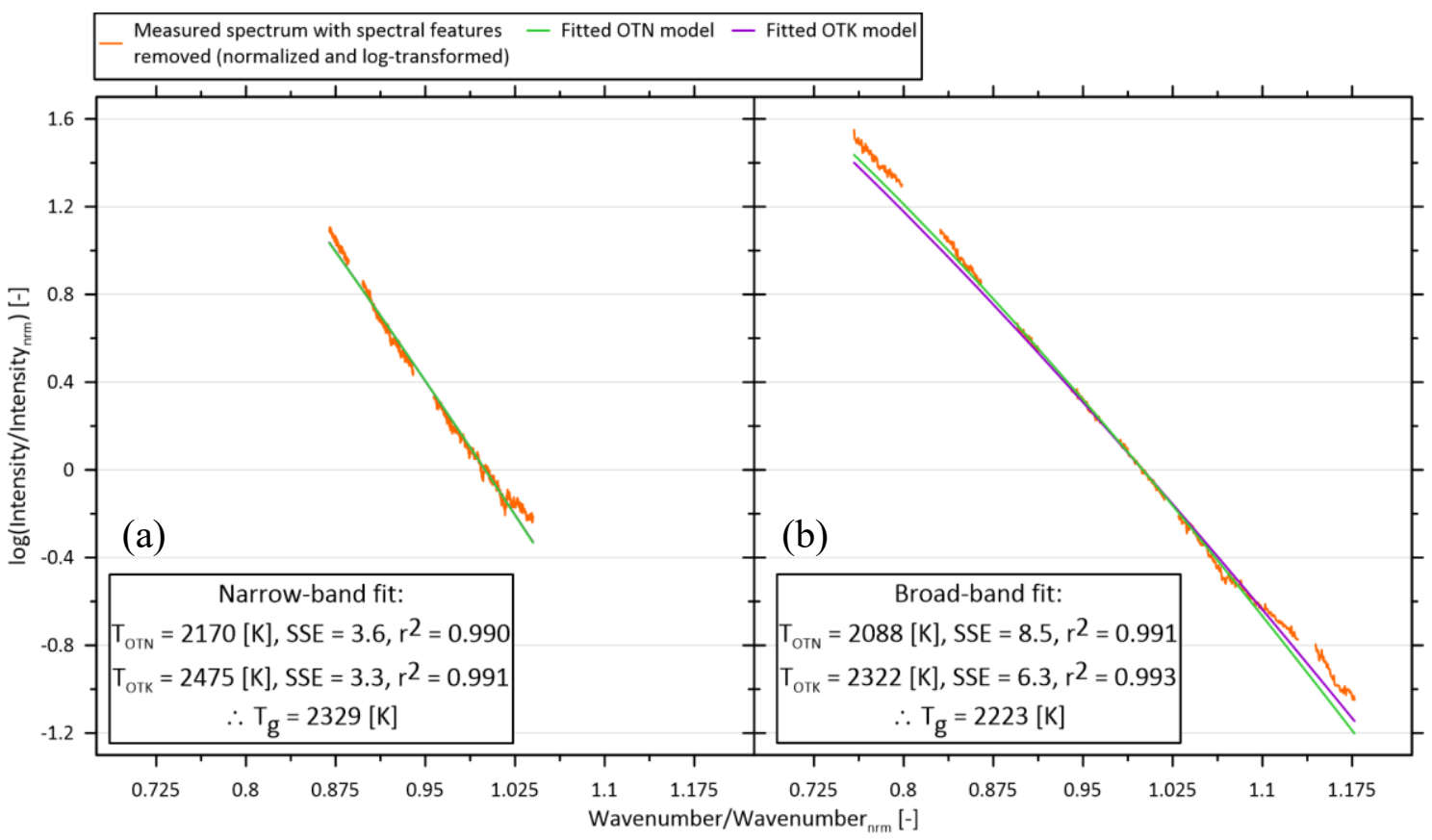

Figure 4.5: Normalized and log-transformed spectra from Figure 4.4 and fitted OTN and OTK models for the (a) narrow-band system and (b) broad-band systems with a $0.05 \mathrm{~mm}$ and $0.01 \mathrm{~mm}$ wide slit, respectively.

The inferred temperatures from each fit are shown in the lower left of each plot along with the coefficient of determination ( $\mathrm{r}^{2}$ value) which was $\geq 0.99$ in all cases for this example. An arbitrary threshold of $\mathrm{r}^{2} \geq 0.99$ was used to filter out noisy or poorly fitting spectra from further analysis. More specifically, if either of the fitted OTN or OTK models had an $\mathrm{r}^{2}$ $<0.99$, that spectrum was discarded. When the fitted OTN and OTK models each had an $\mathrm{r}^{2} \geq 0.99$, an effective temperature, $T_{g}$, was calculated as the inverse sum of squared errors (SSE) weighted average of the two results as presented in Chapter 2 (See Eq. (2.8)). This result is also shown in Figure 4.5 (bottom left corners) for the narrow- and broad-band cases.

For each flare measurement, $T_{g}$ was calculated for every spectrometer reading that passed the preliminary $\mathrm{r}^{2}$ filter. Typically, this produced thousands of instantaneous $T_{g}$ values spanning approximately 5 to 15 minutes in real time, with subsets acquired using various integration times between 1 to $30 \mathrm{~ms}$. Instantaneous $T_{g}$ values often varied by hundreds of degrees due to the continuously changing structure, thickness, and orientation of the flare in the open atmosphere. Further variation might also be attributable to variability in the flowrate or composition of gas being directed to the flare. The distributions of $T_{g}$ closely 
adhered to a normal distribution using either the narrow-band or broad-band systems. A Grubbs test ( $\alpha=0.05$ ) was used as a secondary filter to remove outliers, which removed $\sim 5.5 \%$ of data on average. The mean of the remaining data were used as the effective gas temperature in the flame, $\bar{T}_{g}$.

Figure 4.6 shows the distributions of $\bar{T}_{g}$ using the narrow-band system (blue) and broadband system (orange) for all flares measured during campaigns in North Dakota / southern Saskatchewan (2019) and Ecuador (2020). The independently obtained measurements of $\bar{T}_{g}$ using the narrow-band and broad-band spectrometers generally agreed within a few hundred degrees, but the broad-band results are expected to be more accurate due to their extended wavenumber range representing a larger portion of soot emission's blackbody curve. Therefore, the broad-band $\bar{T}_{g}$ was considered to be the effective flame temperature in subsequent calculations, except for three cases where broad-band spectrometer readings were not acquired and the narrow-band $\bar{T}_{g}$ was used instead.

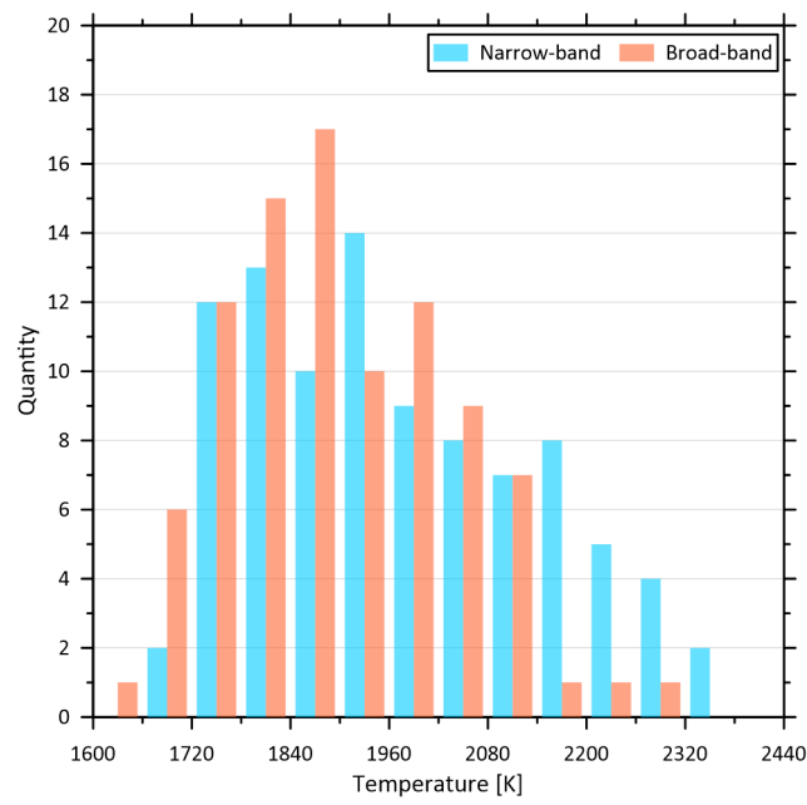

Figure 4.6: The distributions of $\bar{T}_{g}$ using the narrow-band system (blue) and broad-band system (orange) for all flares measured in North Dakota / southern Saskatchewan (2019) and Ecuador (2020). Three flares measured with the narrow-band system were not measured with the broad-band system, and one narrow-band $\bar{T}_{g}$ value of $>2800 \mathrm{~K}$ was considered an outlier and was not included in the narrow-band histogram. 


\subsection{Modeling Potential Emission Signatures of the Na First Resonance Doublet}

The filtered narrow- and broad-band spectra were investigated for potential emission signatures of the first resonance doublets of $\mathrm{Na}$ and $\mathrm{K}$, respectively. Potential emission signatures were first isolated from their background spectrum. The background spectrum for the $\mathrm{Na}$ first resonance doublet is dominated by soot emission; the background of the $\mathrm{K}$ first resonance doublet is similarly dominated by soot emission but is further affected by molecular absorption affects in the $\mathrm{O}_{2} \mathrm{~A}$ band. Peak identification metrics were then developed and used to discern fitted atomic emission signatures of $\mathrm{Na}$ and $\mathrm{K}$ from background levels as further detailed below.

\subsubsection{Isolating Potential Na Emission Signatures and Fitting the Atomic Emission Model}

The background intensity within the Na first resonance doublet wavenumber region (16835 to $17050 \mathrm{~cm}^{-1}$ ) was calculated by interpolating the background spectrum (i.e., the measured spectrum with relevant absorption/emission regions removed) based on the locally weighted regression technique (a.k.a. LOESS) introduced by Cleveland and Devlin (1988). LOESS was preferred to the OTK and OTK models when predicting background spectra because it was more robust; preliminary processing tests found that LOESS accounted for deviations from the exponential trend of soot emission near the $\mathrm{Na}$ first resonance doublet region (due to noise, etc.) better than the exponential OTN and OTK models. In brief, LOESS predicts the intensity at a "center" data point by fitting a weighted second-order polynomial to neighbouring data points and solving the polynomial for the center data point (Cleveland and Devlin 1988). An example of a center data point ("X") and its weighted polynomial (blue) is shown in Figure 4.6 for the $\mathrm{Na}$ first resonance doublet region of narrow-band spectrum and the $\mathrm{K} / \mathrm{O}_{2}$ region of a broad-band spectrum. The weighting curve (magenta) was calculated using the tricubic function described by Cleveland and Devlin (1988). The highest weights were given to the data points nearest the centre data point and weights decreased with absolute wavenumber distance. A constant span value was selected which gave no weight to neighbouring data points farther $|388| \mathrm{cm}^{-1}(13.2 \mathrm{~nm})$ for narrow-band spectra and $|749| \mathrm{cm}^{-1}(41.9 \mathrm{~nm})$ for broad-band spectra. Neighbouring 
data points that were removed (due to being affected by other spectral features) were simply given a weight of zero.

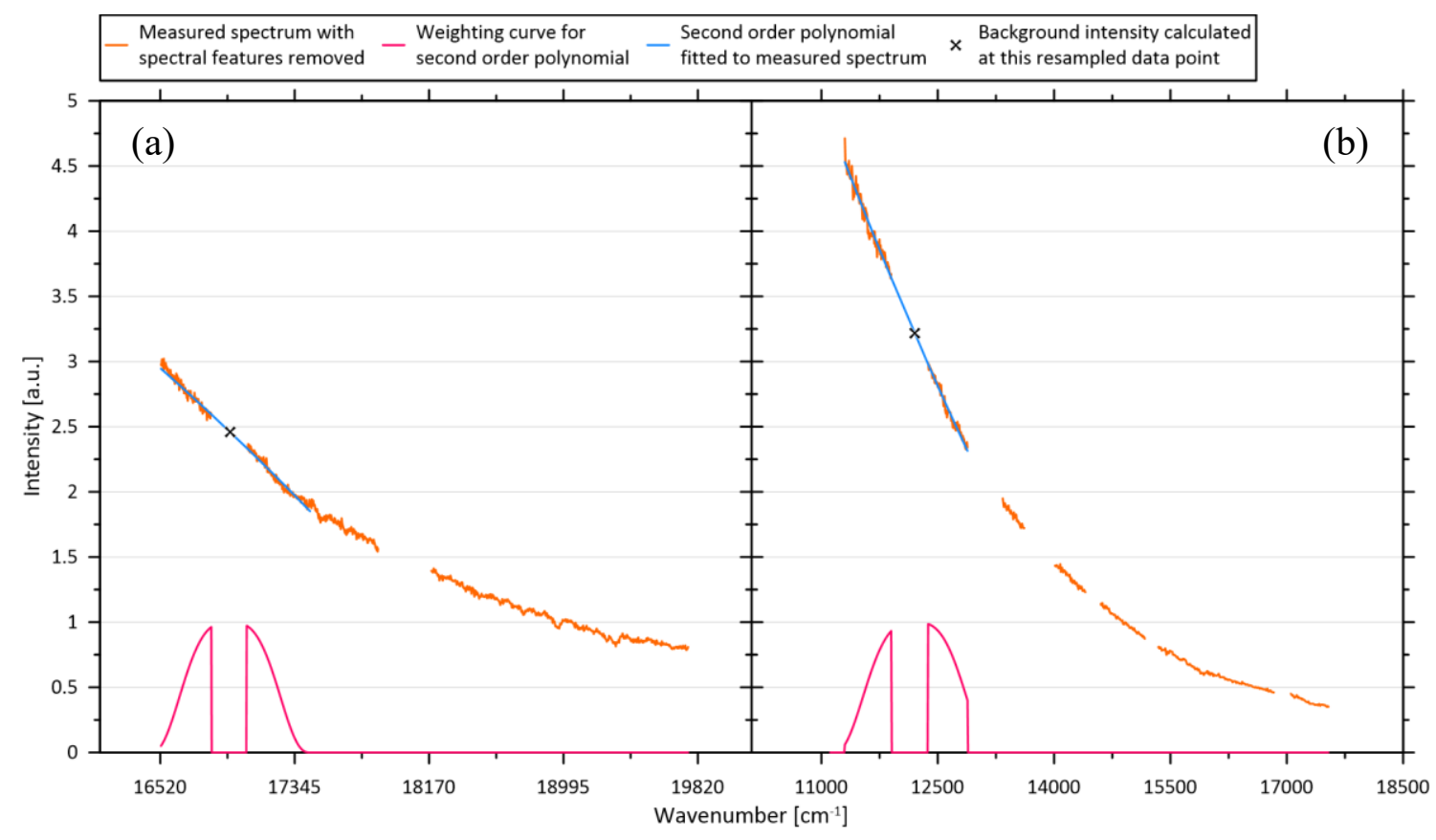

Figure 4.7: Calculating the intensity of a resampled data point using LOESS in the removed data region of the $\mathrm{Na}$ first resonance doublet and the $\mathrm{K} / \mathrm{O}_{2}$ region for the (a) narrow-band spectrum and (b) broad-band spectrum from Figure 4.4, respectively.

The LOESS interpolation was used to calculate the intensity at all resampled data points within the $\mathrm{Na}$ and $\mathrm{K} / \mathrm{O}_{2}$ regions which produced an interpolated background for each region. The interpolated background was subtracted from the measured spectra to isolate the absorption features of interest.

Figure 4.8 illustrates the process to isolate a potential Na peak from the background. The isolated signature in Figure 4.8 (b) was cropped to only include $16910<\tilde{v}<17000 \mathrm{~cm}^{-1}$ and the two vertical yellow represent the theoretical location of the spectral lines associated with the first resonance doublet of $\mathrm{Na}$. In this case, the potential $\mathrm{Na}$ signature is shifted to the left of its theoretical location by approximately $-15 \mathrm{~cm}^{-1}(0.5 \mathrm{~nm}$, further discussed in Figure 4.9) due to the wavelength shift of the spectrometer system as discussed in Chapter 3. This shift is within the expected range of -37.6 to $-14.4 \mathrm{~cm}^{-1}$ based on the pen lamp tests described in Chapter 3. Across all data in the field campaigns, fitted shifts were within -20.2 to $-10 \mathrm{~cm}^{-1}$ for the narrow-band field data. The slight discrepancy between the observed shifts and expected shifts from the pen lamp experiments is not considered 
significant given the inability to fully recreate field conditions during the lamp tests, where in particular, the light from the closely positioned pen style lamp may not fill the systems optics to the same extent as a flare flame.

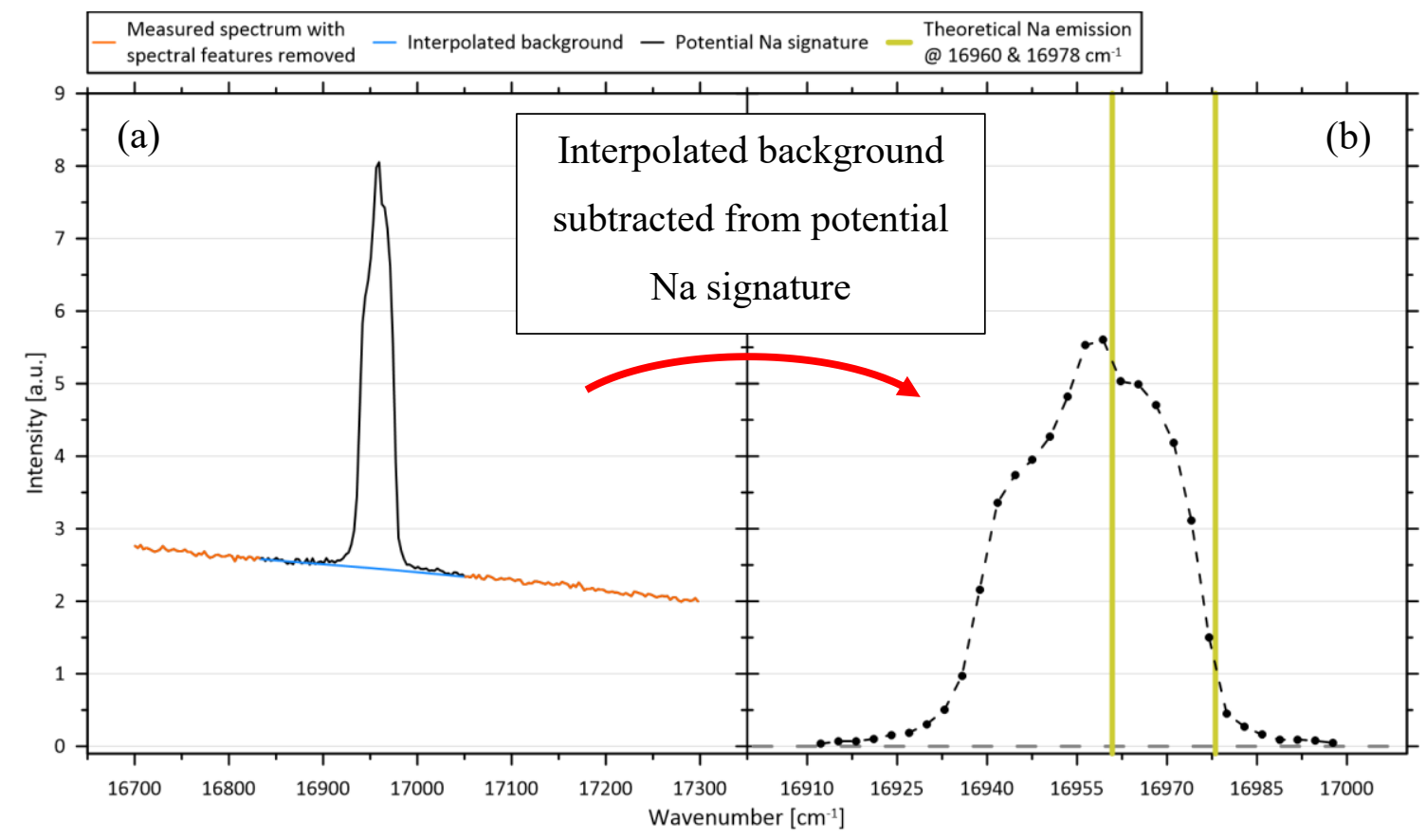

Figure 4.8 (a) The measured narrow-band spectrum from Figure 4.7 with spectral features removed (orange), the interpolated background spectrum generated via the LOESS fit (blue), and a potential

$\mathrm{Na}$ signature within the $\mathrm{Na}$ first resonance doublet region (black). (b) The isolated potential Na signature obtained by subtracting the blue curve from the black in curve (a) and theoretical locations of the spectral lines of the $\mathrm{Na}$ first resonance doublet (yellow).

During a flare measurement, the precise magnitude of wavenumber shift is expected to vary with the motion of the flare flame and the observation angle with respect to the dispersion plane of the spectrometer. This was considered by adding a wavenumber shift term $\left(d \tilde{v}\left[\mathrm{~cm}^{-1}\right]\right)$ to the atomic emission model derived in Chapter 2 which permitted the wavelength shift of individual $\mathrm{Na}$ spectra to be included as a fitting parameter. Similarly, a vertical shift term ( $M$ [a.u.]) was added to allow for a non-zero baseline intensity in measured spectra which could occur when the background spectrum was affected by noise:

$$
I_{R e l}(\tilde{v})=C\left[k_{i_{1}}(\tilde{v}) e^{-4 \ln 2 \frac{\left(\widetilde{v}-\widetilde{v}_{o, i_{1}}-d \widetilde{v}\right)^{2}}{\gamma_{I P}{ }^{2}}}+k_{i_{2}}(\tilde{v}) e^{-4 \ln 2 \frac{\left(\widetilde{v}-\widetilde{v}_{o, i_{2}}-d \widetilde{v}\right)^{2}}{\gamma_{I P}{ }^{2}}}\right]+M
$$

The atomic emission model (Eq. (4.1)) was then fit to the potential Na signature (e.g., Figure 4.8 (b)) via least squares regression. Because field data were corrected by a relative 
calibration, the absolute intensity of the atomic emission model is irrelevant. Consequently, $C$ can be used to scale the atomic emission to model to match the shape of potential Na signatures. Moreover, in-flame parameters such as the effective temperature $\bar{T}_{g}$ and $x_{i}$ are assumed to be uniformly distributed throughout the observed volume of flame (i.e., the observed volume of flame is assumed to be in local thermodynamic equilibrium). Although this is unlikely to be true for a turbulent open-atmosphere flame, this assumption is acceptable since the goal is simply to fit the atomic emission model to the relative intensity of a measured signature for the purpose of species identification rather than to quantify species concentrations.

During the fitting process of the atomic emission model, the terms $C, M, d \tilde{v}$, and $\gamma_{I P}$ were optimized. The parameter $k_{i_{j}}=\sum_{j} S_{j} n x_{i} \phi_{j}$ was calculated following Goldenstein et al. (2017), where the linestrength $\left.\left(S_{j}\left[\mathrm{~cm}^{3} \text { (atom or molecule }\right)^{-1}\right]\right)$ were calculated using Naspecific values from NIST's Atomic Spectra Database (Kramida et al. (2020)) at the measured $\bar{T}_{g}$, an assumed pressure of $101.325 \mathrm{kPa}$, an arbitrary Na volume fraction of $x_{i}=1 \times 10^{-9}[-]$, and a number density $n\left[(\right.$ atom $\left.) \mathrm{cm}^{-3}\right]$ based on ideal gas law at flame conditions. The transition lineshape function $\phi_{j}$ was set to one since it was insignificant relative to broadening from the instrument profile as discussed in Chapter 2. Figure 4.9 superimposes the resulting fit for the measured spectrum of Figure 4.8 (b).

For the example shown in Figure 4.9, the fitted spectrum was shifted $-15 \mathrm{~cm}^{-1}$ from the theoretical location of $\mathrm{Na}$ emission which is within the expected wavelength shift interval of the narrow-band system. Furthermore, the fitted Na model reveals that the potential $\mathrm{Na}$ signature has a full-width half-maximum value of $22 \mathrm{~cm}^{-1}(\sim 0.76 \mathrm{~nm})$, which is very similar to the optical resolution of $0.6 \mathrm{~nm}$ predicted by the manufacturer for the narrowband spectrometer with a $0.05 \mathrm{~mm}$ wide entrance slit. The close fit of the atomic emission model to the measured signature, and the plausible values for wavelength shift and instrument broadening provide convincing evidence this signature belongs to $\mathrm{Na}$. Within this spectral region, the atomic species with the next strongest emission lines is titanium, which produces three relevant lines at 16926.0, 16934.9, and $16946.5 \mathrm{~cm}^{-1}$. However, titanium is not expected to be present in produced water (see Figure 1.2) and the 
corresponding linestrengths are approximately four orders of magnitude less than those of $\mathrm{Na}$ in this region (as estimated using the LineSurvey application provided by Goldenstein et al. (2017) at an electronic temperature of $2300 \mathrm{~K}$ ).

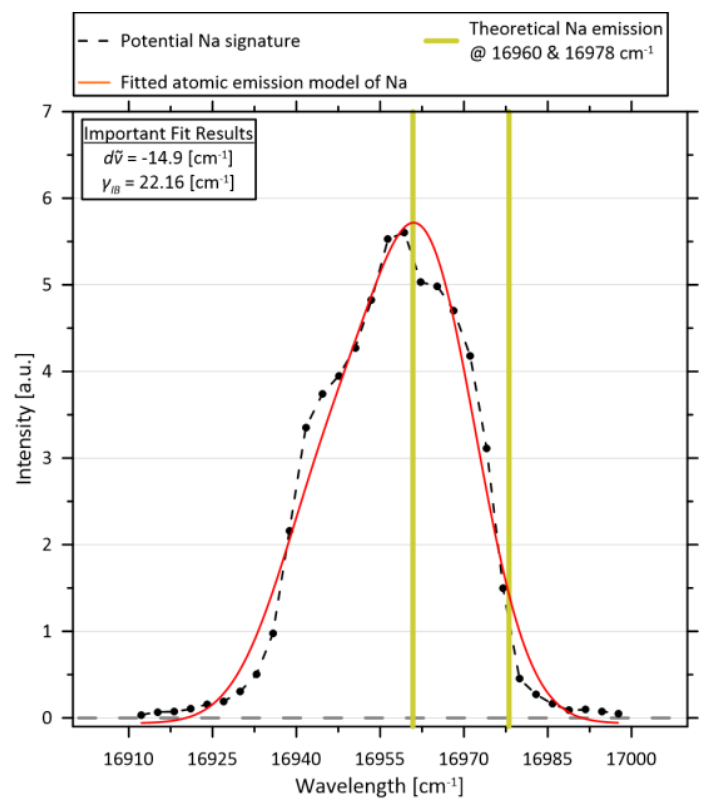

Figure 4.9: Fitted Na atomic emission model overlaid on the measured and isolated spectrum of Figure 4.8.

Presumably, the relatively low intensity and incorrect shape of a measured titanium signature could not be misidentified as sodium, and there exists no other strong emission lines from atomic species in the flame, nor emission bands from molecular species in the flame or its background that could be misidentified as the Na doublet. Thus, the measured signature in Figure 4.9, and all similar cases presented in this thesis, are assumed to be produced by $\mathrm{Na}$ atoms in the flame. As detailed in Section 4.4, candidate peaks like that of Figure 4.9 had to pass a set of three different peak identification metrics prior to being declared a successful detection of $\mathrm{Na}$ or $\mathrm{K}$ in the field.

\subsubsection{Isolating a Potential Emission Signature of $K$ and Fitting the Atomic Emission Model}

Background intensities in the $\mathrm{K}$ emission $/ \mathrm{O}_{2}$ absorption region $\left(12887<\tilde{v}<13333 \mathrm{~cm}^{-1}\right)$ of the broad-band spectra were similarly interpolated using the LOESS technique discussed in Section 4.3.1. Figure 4.10 shows an example of this process. 


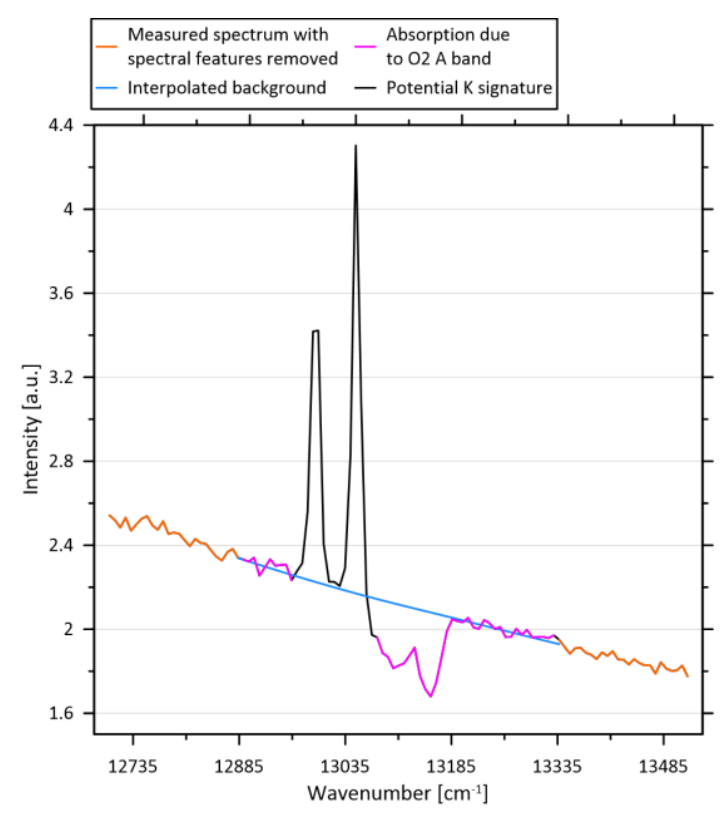

Figure 4.10: The measured broad-band spectrum, which includes K emission region (black) $\mathrm{O2}$ absorption region (magenta), and background radiative emission of the flame (orange), and the LOESS interpolated background through the $\mathrm{K} / \mathrm{O}_{2}$ region (blue).

The interpolated background (blue curve) represents the flare flame's radiative emission prior to being absorbed by ambient $\mathrm{O}_{2}$ (i.e., $I_{o}$ in the molecular absorption model). The measured spectrum - which includes the $\mathrm{K}$ emission region (black), $\mathrm{O}_{2} \mathrm{~A}$ band absorption region (magenta), and background radiative emission outside the $\mathrm{K}$ and $\mathrm{O}_{2}$ regions (orange) - is what is remains after being absorbed by ambient $\mathrm{O}_{2}$ (i.e., the measured spectrum is the transmitted emission, or $I_{t}$ in the molecular absorption model).

The spectral profile of the $\mathrm{O}_{2} \mathrm{~A}$ band (magenta) within measured spectrum was modeled using the molecular absorption model derived in Chapter 2. This permitted potential K signatures within the $\mathrm{O}_{2} \mathrm{~A}$ band region to be isolated and fit by the atomic emission model. Similar to the Na model, parameters for wavelength shift $(d \tilde{v})$ and vertical offset from the interpolated background $(M)$ were included to account for small shifts in the field data :

$$
I_{R e l}(\tilde{v})=D I_{\text {interp }}(\tilde{v}) \sum_{j=1}^{\infty} e^{-k_{i_{j}}(\tilde{v}) L} e^{-4 \ln 2 \frac{\left(\widetilde{v}-\widetilde{v}_{o, i_{j}}-d \tilde{v}\right)^{2}}{\gamma_{I P}{ }^{2}}}+M
$$

The parameters $d \tilde{v}, \gamma_{I P}, M$, and the arbitrary scaling factor $D$ were optimized via a least squares fitting procedure. The resulting fit to the measured $\mathrm{O}_{2}$ absorption profile (the magenta region in Figure 4.10) was used as the background when isolating the potential K 
signature (the black region in Figure 4.10). In the least squared fitting procedure for the molecular absorption model, $k_{i_{j}}$ was evaluated using values found in the HITRAN database (Gordon et al. (2017)) at $T_{a m b}$, an assumed pressure of $101.325 \mathrm{kPa}$, an $\mathrm{O}_{2}$ volume fraction of $x_{i}=0.21[-]$, and a number density $n$ [molecule $\mathrm{cm}^{-3}$ ] based on ideal gas law at ambient conditions. Figure 4.11 superimposes the resulting fit onto the $\mathrm{O}_{2} \mathrm{~A}$ band region in Figure 4.10.

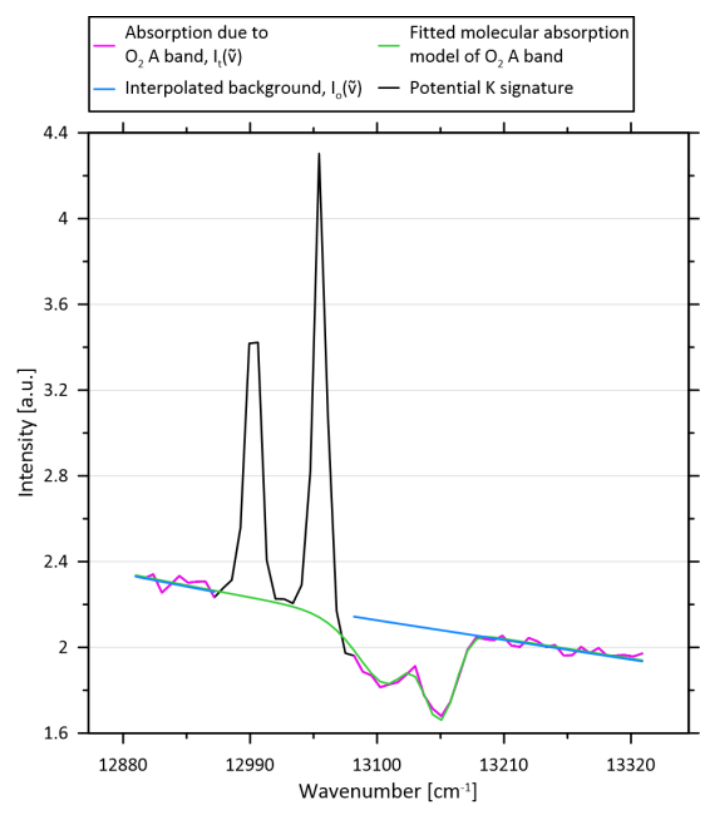

Figure 4.11: The molecular absorption model fitted to the $\mathrm{O}_{2} \mathrm{~A}$ band region in Figure 4.10.

The potential $\mathrm{K}$ emission signature in Figure 4.11 was isolated by subtracting the green curve from the black curve. The result is shown in Figure 4.12 (cropped to $12943<\tilde{v}$ $<13110 \mathrm{~cm}^{-1}$ ) along with the theoretical emission lines of the $\mathrm{K}$ first resonance doublet (yellow), and the fitted atomic emission model (red). The atomic emission model was fit to the isolated potential $\mathrm{K}$ signature using the exact same procedure as the Na signature, instead with K-specific values from NIST's Atomic Spectra Database (i.e., Kramida et al. (2020)). 


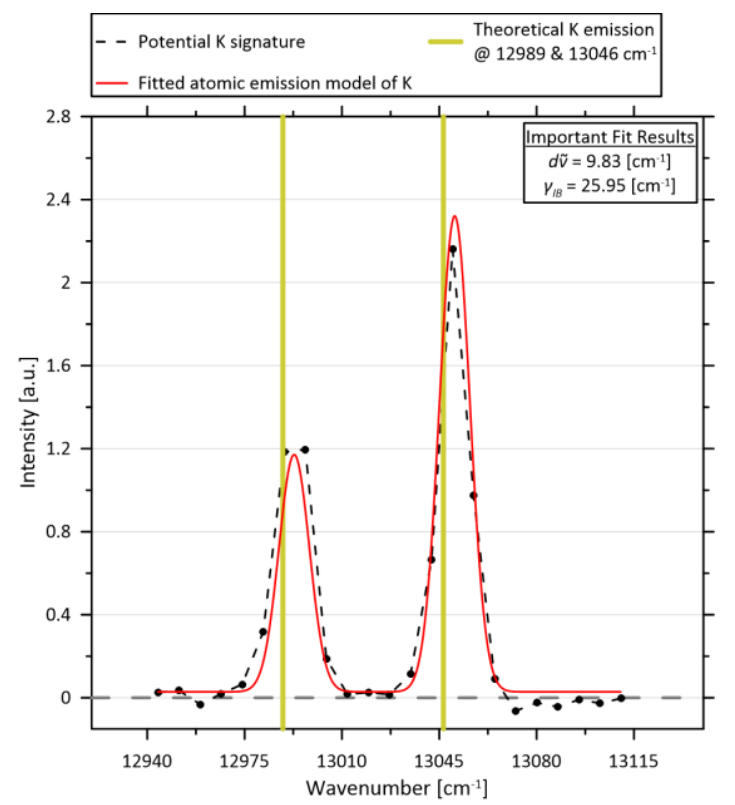

Figure 4.12: The atomic emission model fitted to the isolated K spectrum from Figure 4.11. Yellow lines indicated the theoretical locations of the $\mathrm{K}$ emission peaks.

The fit results in Figure 4.12 indicate that the $\mathrm{K}$ spectrum was shifted $+4 \mathrm{~cm}^{-1}$, which is within the expected wavelength shift interval of the broad-band system (presented in Chapter 3). Furthermore, the $13 \mathrm{~cm}^{-1}(\sim 0.77 \mathrm{~nm})$ full-width half-max of the fitted atomic emission model was similar to the optical resolution of $1 \mathrm{~nm}$ predicted by the manufacturer for broad-band spectrometer with a $0.01 \mathrm{~mm}$ wide entrance slit. Across all broad-band data collected in the field, shifts of -1.3 to $+20.2 \mathrm{~cm}^{-1}(-1.2$ to $+0.1 \mathrm{~nm})$ were observed, which are similar to the estimated range of -10.1 to $+11.9 \mathrm{~cm}^{-1}(-0.7$ to $+0.6 \mathrm{~nm})$ expected from the lamp tests in Chapter 3. The 6 of 29 detected $\mathrm{K}$ signatures whose fitted atomic emission models were shifted $>10 \mathrm{~cm}^{-1}$ were manually reviewed and the $\mathrm{K}$ emission models were confirmed to accurately fit the data.

The close fit between the model and measured signature in Figure 4.12, and the accompanying fit results provide convincing evidence this signature belongs to $\mathrm{K}$. The atomic species with the next strongest emission lines within the spectral region shown in Figure 4.12 is barium, which has a single relevent line at $13030.7 \mathrm{~cm}^{-1}$. While barium is found in produced water, its single emission line will produce an obviously different spectrum than that of the $\mathrm{K}$ doublet. Furthermore, the linestrength of barium in this region is approximately two orders of magnitude less than those of the $\mathrm{K}$ doublet (as estimated using the LineSurvey application provided by Goldenstein et al. (2017) at an electronic 
temperature of $2300 \mathrm{~K})$. Once again, there are no other atomic emission lines or molecular emission bands generated from the flare flame (or the sky/tree background) that could be mistaken for the $\mathrm{K}$ doublet. Thus, the measured signature in Figure 4.12, and all similar cases presented in this thesis, are assumed to be generated by gaseous $\mathrm{K}$ in the flare flame.

\subsection{Peak Identification Metrics}

To facilitate unbiased analysis of the presence of $\mathrm{Na}$ or $\mathrm{K}$ signatures, three different peak identification metrics were considered: the signal to noise ratio, the integrated intensity ratio, and a sum of squares $\mathrm{F}$ test. A potential emission signature needed to satisfy all three metrics to be classified as a successful detection.

\subsubsection{The Signal to Noise Ratio Peak Identification Metric}

The peak intensities of potential $\mathrm{Na}$ and $\mathrm{K}$ signatures within the spectral region of interest (i.e., the black regions in Figure 4.13) were compared with the standard deviation $(\sigma)$ of the intensity of the measured signal within adjacent regions, represented by the green curves in Figure 4.13. The LOESS technique described in Section 4.3.1 was used to estimate the smooth blue background in Figure 4.13. The blue curve was subtracted from the green curve to produce background intensities which were used to calculate $\sigma$. A single $\sigma$ was calculated for each signature by considering the background intensities from the separate green regions as one single region. 


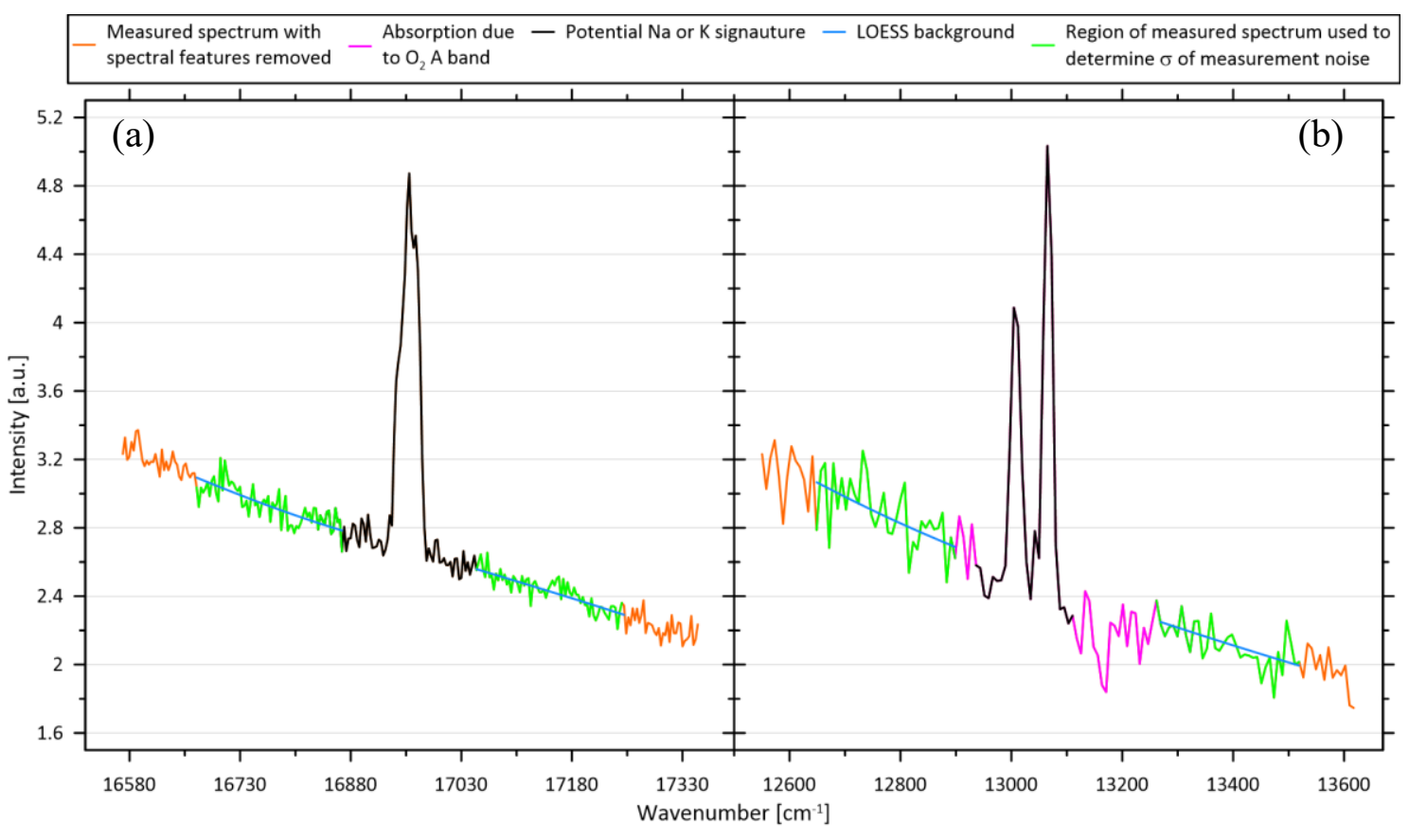

Figure 4.13: The spectral regions from which standard deviation was calculated for a potential emission signature of (a) $\mathrm{Na}$ and (b) $\mathrm{K}$.

The wavenumber intervals that define the green regions in Figure 4.13 are summarized in Table 4.5. To pass this first peak identification metric, the peak intensity of a potential $\mathrm{Na}$ signature had to be $>3 \sigma$. For a potential $\mathrm{K}$ signature to pass, both peak intensities had to be $>3 \sigma$.

Table 4.5: The wavenumber ranges that are used to calculate the standard deviation of measured intensity near the $\mathrm{Na}$ and $\mathrm{K}$ signatures.

\begin{tabular}{|l|l|l|l|}
\hline Species & $\begin{array}{l}\text { Lower Wavenumber } \\
\text { Region for } \sigma\end{array}$ & $\begin{array}{l}\text { Higher Wavenumber } \\
\text { region for } \sigma\end{array}$ & $\begin{array}{l}\text { Amount of Data } \\
\text { Points in Region }\end{array}$ \\
\hline $\mathrm{Na}$ & $\begin{array}{l}17050-17250 \mathrm{~cm}^{-1} \\
(579.7-586.5 \mathrm{~nm})\end{array}$ & $\begin{array}{l}16670-16870 \mathrm{~cm}^{-1} \\
(592.8-599.9 \mathrm{~nm})\end{array}$ & $136(68 \times 2)$ \\
\hline $\mathrm{K}$ & $\begin{array}{l}13262-13520 \mathrm{~cm}^{-1} \\
(739.6-754 \mathrm{~nm})\end{array}$ & $\begin{array}{l}12648-12900 \mathrm{~cm}^{-1} \\
(775.2-790.6 \mathrm{~nm})\end{array}$ & $68(34 \times 2)$ \\
\hline
\end{tabular}

\subsubsection{The Integrated Intensity Ratio Peak Identification Metric}

The second metric used in the peak identification process was an integrated intensity ratio, which assessed whether a potential emission signature contained greater power relative to nearby regions within the measured spectrum. As illustrated in Figure 4.14, a potential peak was first isolated by subtracting the LOESS interpolated background (blue) from the measured spectra (yellow) to produce the wide isolated peak spectra (black). For the K 
peaks in the broad-band data (Figure $4.14(\mathrm{~d})$ ), the effects of molecular absorption of $\mathrm{O}_{2}$ were then removed by subtracting the fitted $\mathrm{O}_{2}$ model (magenta) from the isolated $\mathrm{K}$ peak region. The resulting increase in the height of the $\mathrm{K}$ peaks is faint but apparent when comparing Figure 4.14 (d) with Figure 4.15 (b).

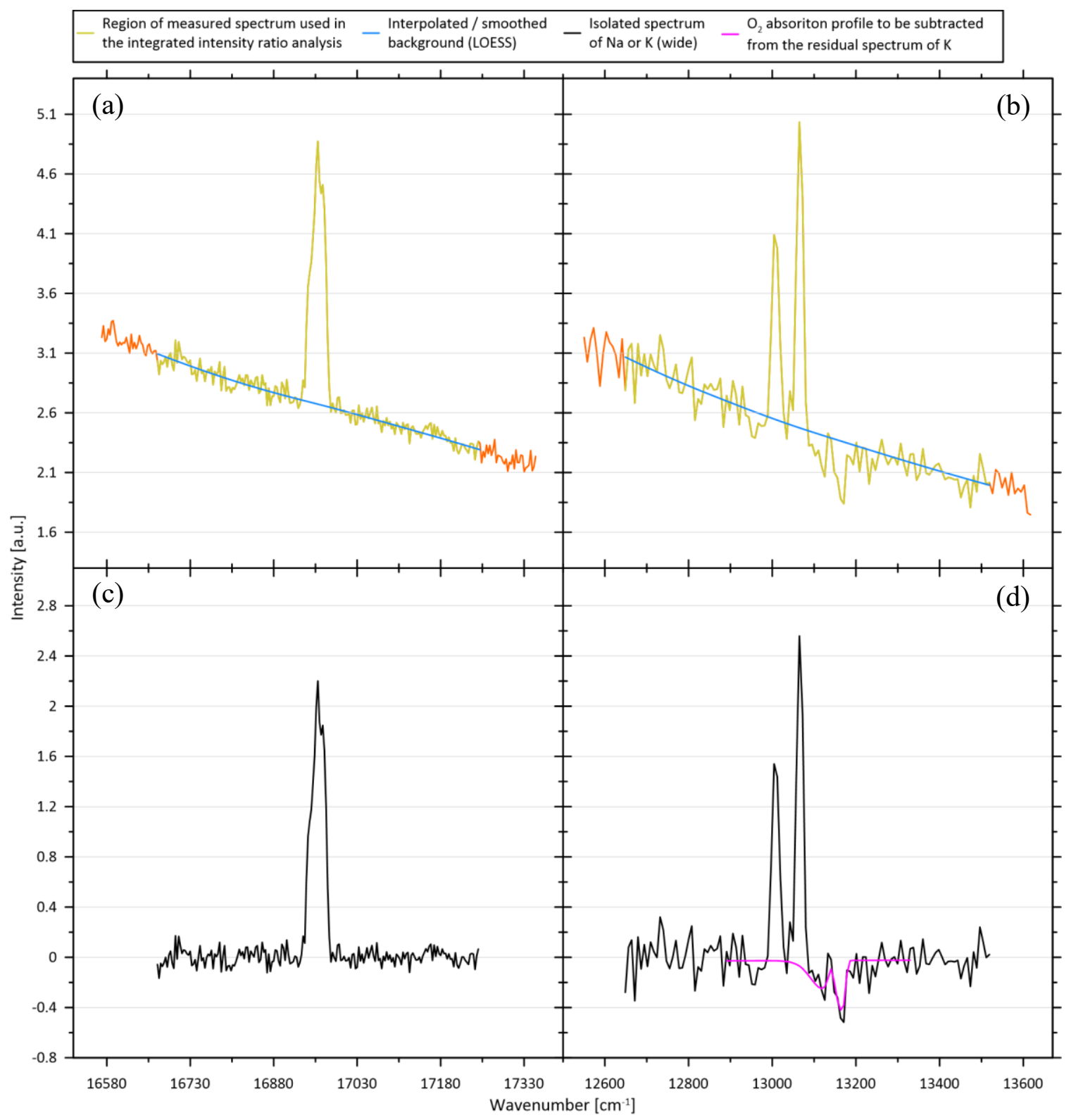

Figure 4.14: (a) and (b) The region of measured spectrum that is included in the integrated intensity ratio analysis (the yellow curve) which had its background subtracted (the blue curve) for $\mathrm{Na}$ and $\mathrm{K}$, respectively. (c) and (d) The isolated spectrum of $\mathrm{Na}$ and $\mathrm{K}$ (wide), respectively. The fitted $\mathrm{O}_{2}$ absorption model (the magenta curve) is overlaid onto (d). 
The integrated intensity value for a candidate peak was calculated as the area under a fixed width "window" centered about the peak (see the red curves and red areas in Figure 4.15). This was compared with the integrated intensities for equivalent width regions away from the peaks (see the orange curves and orange areas in Figure 4.15). The width of the integration regions for the $\mathrm{Na}$ and $\mathrm{K}$ peaks are summarized in Table 4.6.

Table 4.6: Window widths used to calculate the integrated area ratio for $\mathrm{Na}$ and $\mathrm{K}$ signatures.

\begin{tabular}{|l|l|}
\hline Species & Window Length \\
\hline $\mathrm{Na}$ & $\begin{array}{l}16910-17000 \mathrm{~cm}^{-1} \\
(588.2-591.4 \mathrm{~nm})\end{array}$ \\
\hline $\mathrm{K}$ & $\begin{array}{l}12937-13115 \mathrm{~cm}^{-1} \\
(762.5-773 \mathrm{~nm})\end{array}$ \\
\hline
\end{tabular}

To calculate integrated intensities in adjacent regions for comparison with the peak, the fixed width window was stepped across the spectrum (black curves in Figure 4.15) in $1 \mathrm{~cm}^{-1}$ increments, and the area was calculated at each step (shown for an example location in orange in Figure 4.15). The integrated intensity ratio was calculated as the ratio of the area under the window centered on the potential emission signature divided by the largest integrated area calculated from all adjacent windows that did not overlap the potential emission signature. Importantly, negative intensity values were set to zero during the integration. To be further considered as detections, potential $\mathrm{Na}$ and $\mathrm{K}$ signatures had to have an integrated intensity ratio $>1.22$ and $>0.9$, respectively. 


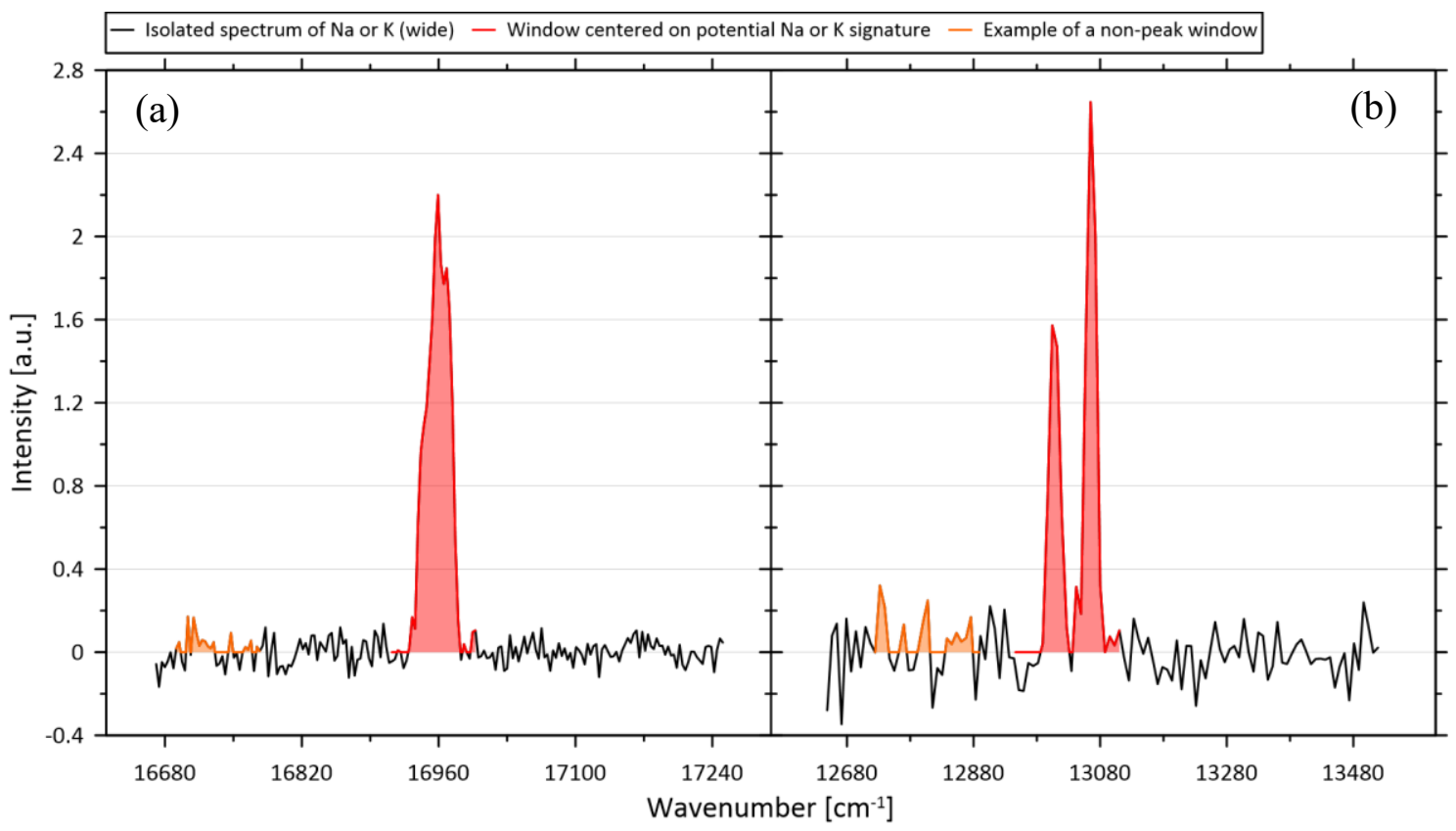

Figure 4.15: Example of the integrated intensity ratio calculation. The portions of the spectra from Figure 4.14 (c) and $d$ that are contained within the peak window and a non-peak window are defined by the coloured lines. The shaded area under coloured lines represents the integrated intensity for those windows.

\subsubsection{The F test Peak Identification Metric}

The third peak metric used was an F test, which is a statistical hypothesis test used to compare the SSE between an advanced and simple model fitted to the same dataset (Motulsky and Christopoulos 2003). In this case, the advanced model was the atomic emission model and the simple model was a line constrained to $\mathrm{y}=0$. A line was used for the simple model because it describes an isolated potential emission signature that contains no atomic emission. The $\mathrm{F}$ test was included in the peak identification metric to determine whether the relative difference in SSE between the atomic emission model and the line fitted to a suspect emission signature was larger than what could be expected by chance due to the added complexity (i.e., the fitted parameters) of the atomic emission model. In other words, from Motulsky and Christopoulos (2003), "(t)he P value [obtained from the F test] answers this question: If the (simple model) is really correct, in what fraction of experiments will the difference in the sum of squares be as large as (what) was observed or larger?"

The F test was used to test the null and alternative hypotheses: 


$$
\begin{gathered}
H_{o}=\text { The simple model fits the potential emission signature best } \\
H_{a}=\text { The advanced model fits the potential emission signature best }
\end{gathered}
$$

where the simple model (line constrained to $y=0$ ) had zero optimized parameters and the advanced model (the atomic emission model) had four optimized parameters. An alpha of 0.01 was chosen to determine significance instead of the typical value of 0.05 in an effort to reduce the probably of falsely accepting the alternative hypothesis (i.e., to reduce type 1 error).

Obtaining a $\mathrm{P}$ value from an $\mathrm{F}$ test requires the $\mathrm{F}$ ratio to be calculated first (Motulsky and Christopoulos 2003):

$$
F=\frac{\left(S S E_{1}-S S E_{2}\right) D F_{2}}{\left(D F_{1}-D F_{2}\right) S S E_{2}}
$$

where the subscripts 1 and 2 represent the simple and advanced model respectively, and $S S E$ and $D F$ are the sum of squared error and the degrees of freedom for the given model, where according to Motulsky and Christopoulos (2003):

$$
\begin{aligned}
& D F_{1}=N-P_{1} \\
& D F_{2}=N-P_{2}
\end{aligned}
$$

where $P$ is the number of parameters optimized by regression, and $N$ is the number of data points fit by the models. The $\mathrm{F}$ ratio is found in a cumulative $\mathrm{F}$ distribution with $D F_{2}-$ $D F_{1}$ and $D F_{2}$ degrees of freedom, which produces a right-tailed $\mathrm{P}$ value (Motulsky and Christopoulos 2003).

\subsubsection{Identifying Successful Vs. Null Detections via the Peak Identification Metrics}

To reduce computational burden, the peak identification metrics were used to analyse the strongest signatures of $\mathrm{Na}$ and $\mathrm{K}$ from each flare measurement, which were manually selected. Manual selection involved sorting the signatures in descending order according to their fitted $C$ value from Eq. (4.1), which represents the scaled height of the peak. Up to three of the tallest candidate peaks were processed for each flare measurement, where 
the goal was to identify at least one successful detection of $\mathrm{Na}$ and $\mathrm{K}$ (if present) indicating the presence of that species in the flare flame.

The manually selected potential $\mathrm{Na}$ and $\mathrm{K}$ emission signatures were declared a successful detection when the signal to noise ratio, integrated area ratio, and $\mathrm{P}$ value from the $\mathrm{F}$ test simultaneously met the thresholds presented in Table 4.7

Table 4.7: Signal to noise ratio, integrate intensity ratio, and $P$ value (from the $F$ test) thresholds defining successful detections of $\mathrm{Na}$ and $\mathrm{K}$.

\begin{tabular}{|l|l|l|l|}
\hline Species & Signal to Noise Ratio [-] & Integrated Intensity Ratio [-] & P Value (F test) \\
\hline $\mathrm{Na}$ & $>=3$ & $>=1.22$ & $<0.01$ \\
\hline $\mathrm{K}$ & $>=3$ (for each peak) & $>=0.9$ & $<0.01$ \\
\hline
\end{tabular}

For illustration purposes, Figure 4.16 shows borderline successful and null detections of $\mathrm{Na}$ emission signatures. Similar borderline successful and null detections of $\mathrm{K}$ are shown in Figure 4.17. These figures contain emission signatures measured during the 2019 campaign in North Dakota / southern Saskatchewan and the 2020 campaign in Ecuador. The left and right columns in these figures represent successful and null detections, respectively. Furthermore, the signatures in these figures are sorted in descending order according to their integrated intensity ratio, starting from the top left and working towards the bottom right. It is important to understand that these examples show the most ambiguous cases of the campaigns. Successful detection signals more typically looked like Figure 4.15 (a) and (b). 


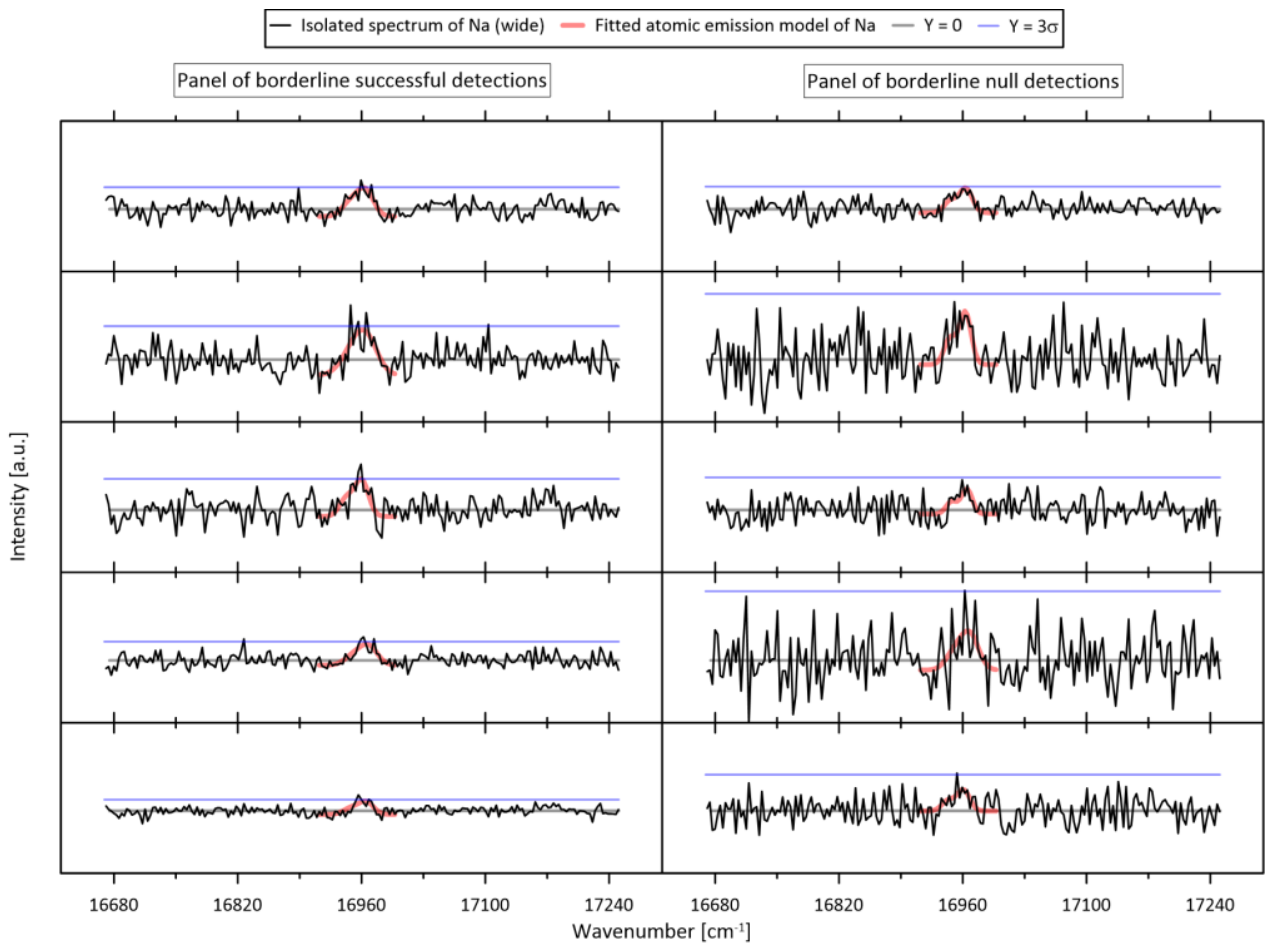

Figure 4.16: Example spectra showing borderline successful (left panel) and null (right panel) detections of $\mathrm{Na}$ signatures in field data acquired during the 2019 field measurement campaign in North Dakota / Southern Saskatchewan and the 2020 field measurement campaign in Ecuador.

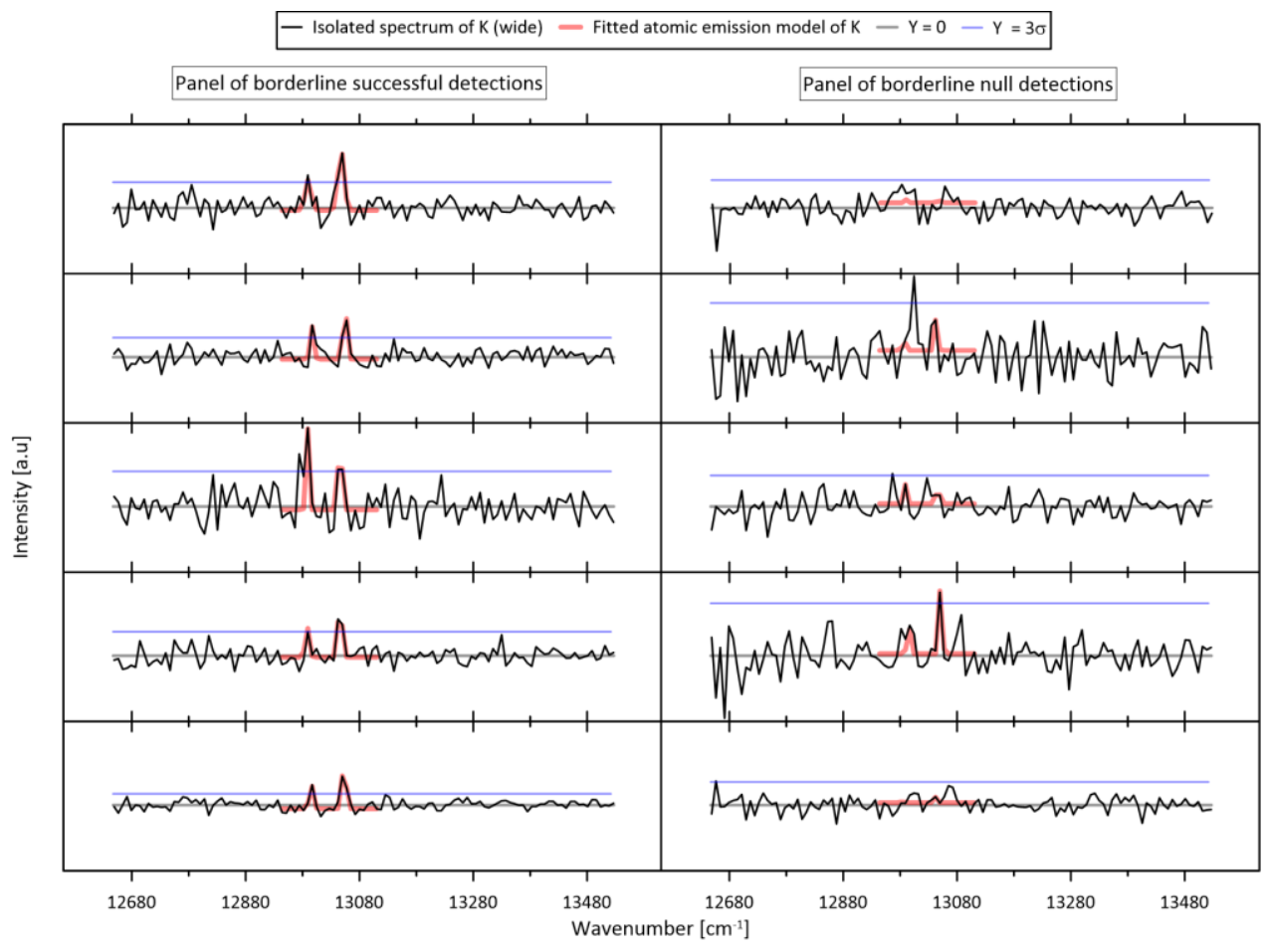

Figure 4.17: Example spectra showing borderline successful (left panel) and null (right panel) detections of $\mathrm{K}$ signatures in field data acquired during the 2019 field measurement campaign in North Dakota / Southern Saskatchewan and the 2020 field measurement campaign in Ecuador. 


\section{Chapter 5 Results and Discussion}

Spectral emission measurements were completed at 95 distinct upstream oil and gas (UOG) sector flares during two separate measurements campaigns. Eighty-one flares were measured during a November 2019 campaign in the Bakken region of Williston Basin in northwest North Dakota (76) and southeast Saskatchewan (5), and 14 flares were measured during a February 2020 campaign in the amazon region of Ecuador. This chapter presents analyzed results on the frequency and regional variations in detection of $\mathrm{Na}$ and $\mathrm{K}$ atomic emission signatures in flare flames and the implications for the likelihood of liquid carryover into flare systems. Effective flame temperature data are also discussed. Finally, using publicly available production and well data for North Dakota, a statistical analysis was completed to investigate potential correlations of liquid carry-over with well age and production and flaring volumes.

\subsection{The Prevalence of Liquid Carry-Over Amongst the Flares Measured in North Dakota, southern Saskatchewan, and Ecuador}

The data processing techniques and peak identification metrics presented in Chapter 4 were used to identify flares that had at least one successful detection of either $\mathrm{Na}$ or $\mathrm{K}$ atomic emission signatures in the flame spectral emission. Successful detections under these criteria confirmed that $\mathrm{Na}$ and/or $\mathrm{K}$ were present in the flame at the time of measurement. In the absence of other known means to introduce $\mathrm{Na}$ or $\mathrm{K}$ into the flare gas, this was considered proof of liquid carry-over of $\mathrm{Na}$ and/or K-laden aerosols into the flare system. As summarized in Table 5.1, 72\% (68 of 95) flares in this study had evidence of either $\mathrm{Na}$ or $\mathrm{K}$ signatures. Moreover, these species were detected in flares at all locations in this study. This suggests that liquid carry-over into flare systems is common at UOG production sites. 
Table 5.1: The amount of flares found to have evidence of liquid carry-over in North Dakota, southern Saskatchewan, and Ecuador.

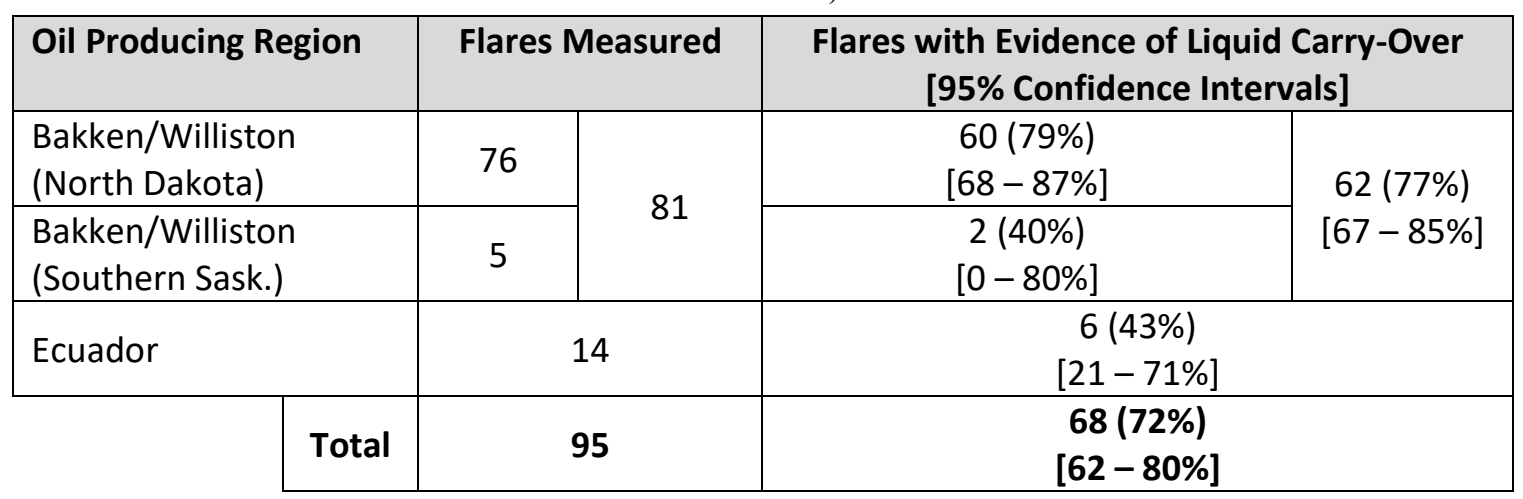

The bias-corrected and accelerated bootstrapping technique described in Efron and Tibshirani (1994) was used to determine the 95\% confidence intervals of the population's mean detection rate from the flare sample data. The square brackets in Table 5.1 show the confidence intervals for each sample and region. The present results suggest that 67 to $85 \%$ of flares in the Bakken region of the Williston basin (including southern Saskatchewan and North Dakota) were experiencing some amount of liquid carry-over. Considering also flares measured in Ecuador, if these regions are representative of flares more broadly, then between 62 to $80 \%$ of upstream flares are likely to be burning some amount of entrained salt species. Given ongoing research highlighting adverse impacts of entrained salts on black carbon, $\mathrm{CO}$, and VOC emissions from simulated flare flames (e.g., Roth et al. 2020), these results have profound implications for our understanding of the potential impacts of flare emissions globally.

Figure 5.1 shows the percentage of flares with successful detections of $\mathrm{Na}, \mathrm{K}$, or both species. Data are plotted separately for the Williston basin region (i.e., North Dakota / southern Saskatchewan) and Ecuador as well as for all measured flares in the study. 


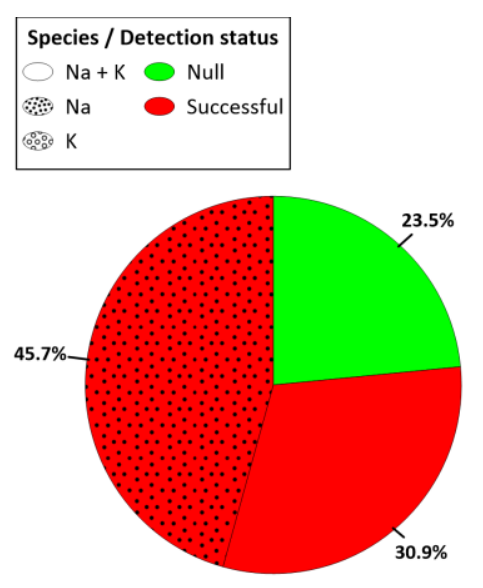

North Dakota / Southern Sask. 2019

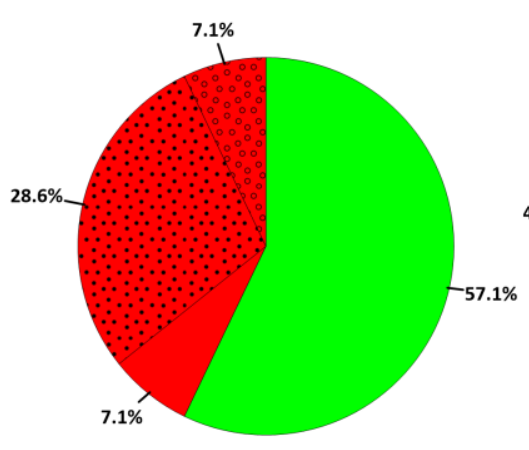

Ecuador 2020

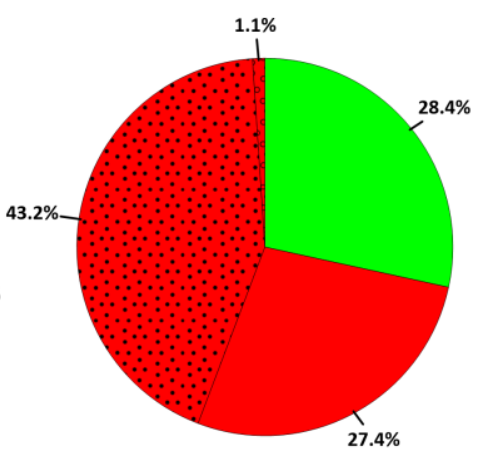

Combined total

Figure 5.1: The breakdown of detection status (by species) for flares measured in North Dakota / southern Saskatchewan in November 2019, Ecuador in February 2020, and their combined total.

Considering all measured flares in this study, Figure 5.1 shows that $\mathrm{Na}$ and $\mathrm{K}$ was present in the flames of $\sim 71 \%$ and $\sim 32 \%$ of flares, respectively. The rate of successful $\mathrm{Na}$ detections is $\sim 2 \times$ the rate of successful $\mathrm{K}$ detections, which aligns with the spectrometer sensitivity measurements that were discussed in Chapter 3 (see Section 3.5.1). The plot of the typical produced water components for the Williston basin presented in Chapter 1 (See Figure 1.2) shows that concentrations of $\mathrm{Na}^{+}$and $\mathrm{K}^{+}$could vary over two orders of magnitude, but were always non-zero. Therefore, if one species was detected by the spectrometer system, it is reasonable to assume that the other was present in some non-zero concentration regardless of its detection status.

Lab-scale experiments by (Kazemimanesh et al. 2020; Jefferson 2017; Roth et al. 2020) have demonstrated a range of adverse effects of entrained $\mathrm{NaCl}$ and $\mathrm{KCl}$ solutions on flare emissions. These may include but are not limited to increasing both the amount and the light absorption potential (i.e., climate warming potential) of emitted soot by a factor of up to $\sim 3 \times$, and significantly increasing benzene (a carcinogen), carbon monoxide (a criteria air contaminant), and unburned fuel emissions (which contribute to volatile organic compound (VOC) emissions). These effects are thought to depend on the salt loading rate of the flare flame (Roth et al. 2020; Jefferson 2017), which is the ratio of the mass of salt to the mass of the fuel entering the combustion zone. Unfortunately, the salt loading rate could not be determined in the field using the spectrometer system, but experiments are 
being developed to generate an empirical salt loading rate calibration. Ongoing research by Roth et al. (2020) is also seeking to determine threshold salt loading rates at which $\mathrm{NaCl}$ and $\mathrm{KCl}$ aerosols begin to notably affect solid and gas phase emissions of a lab-scale flare flame. Thus, although the present results now confirm that $\mathrm{Na}$ and $\mathrm{K}$ is readily and frequently detectable in flare flames under field conditions, further research will be required to understand the associated emissions impacts.

Figure 5.2 and Figure 5.3 map the locations of measured flares in the Williston basin and Ecuador respectively, where the half-circle symbols indicate whether $\mathrm{Na}, \mathrm{K}$, or both species were detected. Measurements of both species were attempted at all except three locations where broad-band data to measure $\mathrm{K}$ were not acquired. These are indicated by the white, right half circles (two locations in Figure 5.2 and one in Figure 5.3). A single flare North Dakota (Figure 5.2) was measured twice, once in June 2018 during a preliminary scouting campaign and again in November 2019, and is marked by a star icon. For this flare $\mathrm{Na}$ was successfully detected in both 2018 and 2019, which anecdotally suggests that liquid carry-over may be a reoccurring or consistent problem for some flare systems. The locations of successful $\mathrm{Na}$ detections in Figure 5.2 appear to be evenly distributed throughout the region with no particular pattern (a pattern might indicate there are regional differences in produced water composition, the size or type of on-site separation equipment used, etc.). Potential drivers of liquid carry-over such as well age and monthly production volumes are considered Section 5.3 using publicly accessible production and well data for North Dakota. 


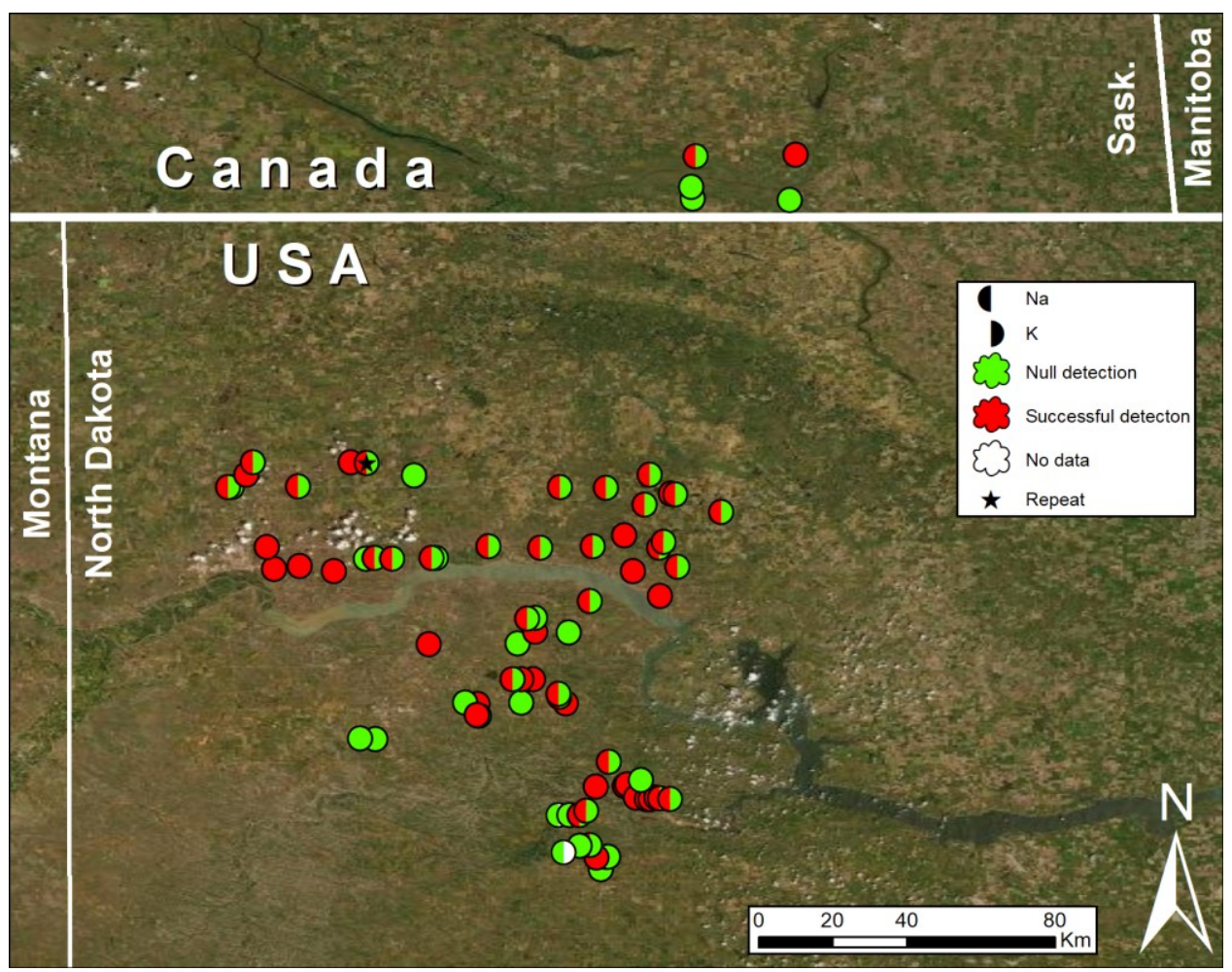

Figure 5.2: The location and detection status of the flares measured in North Dakota and southern Saskatchewan in November 2019.

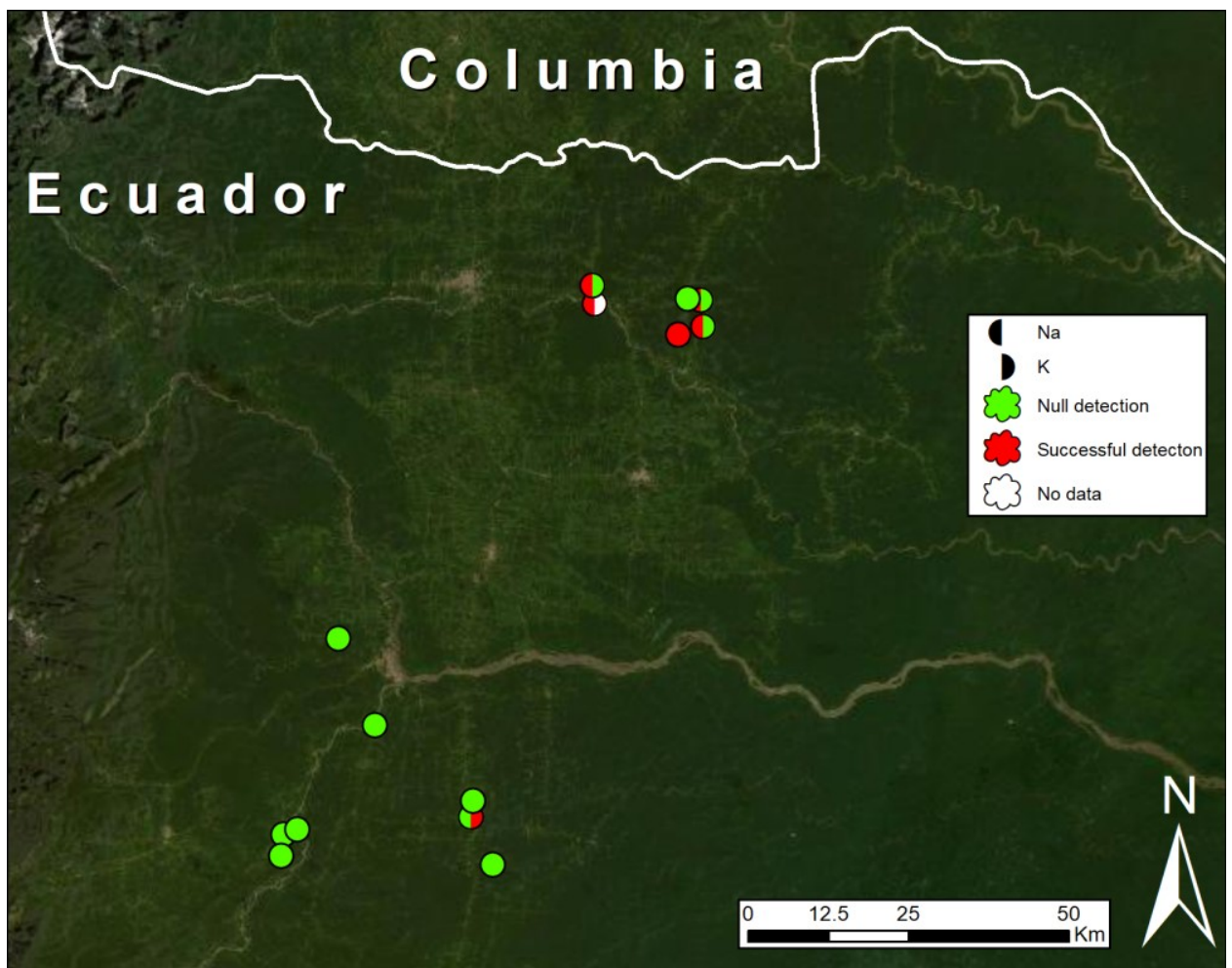

Figure 5.3: The location and detection status of the flares measured in Ecuador in February 2020. 
Although the sample of 14 measured flares in Ecuador is small, the data in Figure 5.3 do suggest some spatial variation in the probability of detection. In the northern region of the map, four of six measured flares had either a $\mathrm{Na}$ or $\mathrm{K}$ detection, where as only one of eight flares in the more southern region had a successful detection. This preliminarily suggests that liquid carry-over may vary with location, where factors including mean well production volumes, types of separation equipment/infrastructure, or even groundwater composition are all plausible explanations for a locational dependency. However, the small sample size precludes any general conclusions.

\subsection{Flame Temperature Comparison for Flares with/without Detections}

The distribution of effective flame temperature $\left(\bar{T}_{g}\right)$ for flares with and without a positive detection are shown in Figure 5.4. Three flares (one with a positive detection and two without) are not included in Figure 5.4 because spectrometer readings were not recorded with the broad-band system. A two-tailed t-test on the $\bar{T}_{g}$ distributions produced a $\mathrm{p}$-value of 0.23 (i.e., >0.05), meaning that the population means of $\bar{T}_{g}$ for flares with and without a positive detection are not statistically different.

The presence of chlorine in flare flames with a successful detection (via $\mathrm{NaCl}$ or $\mathrm{KCl}$ ) suggests there could be a possibility of forming chlorinated species such as dioxins or furans in the flare emissions. Altarawneh et al. (2009) states that the formation pathways of these toxic species have a temperature ceiling of $\sim 1073 \mathrm{~K}$. In comparison, the lowest $\bar{T}_{g}$ of a flare with a successful detection was $>1650 \mathrm{~K}$. This suggests dioxins and furans are unlikely to form in/near the combustion zone of flare flames, but could potentially still be formed in their cooled post-flame region. Given the impacts of chlorinated species emissions for people and environment, understanding their formation potential in flares is one of the many goals of the ongoing research by Roth et al. (2020). 


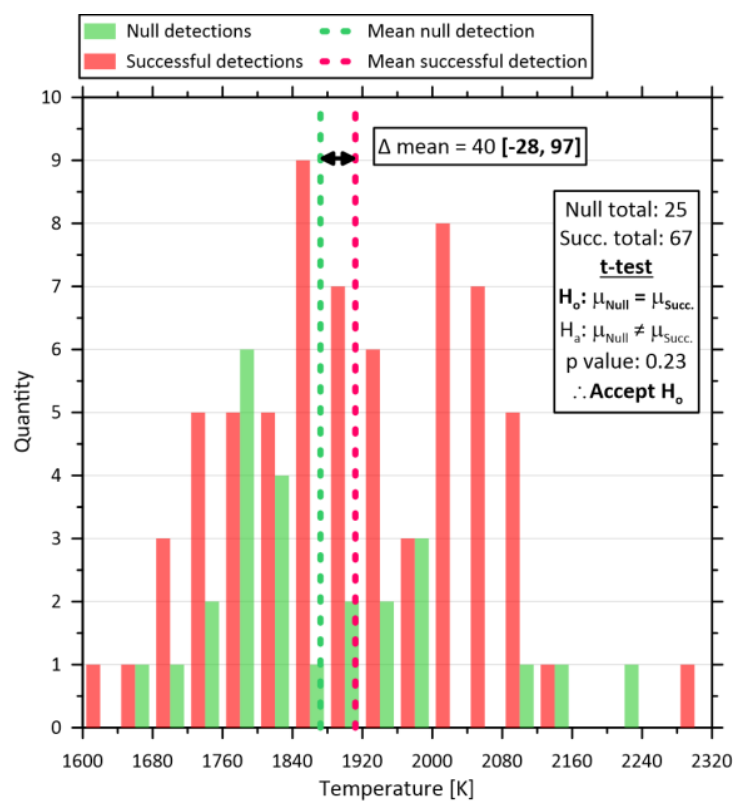

Figure 5.4: The effective flame temperature distributions for the flares with and without successful detections including a two tailed t-test comparing the mean flame temperature of these distributions.* Excludes three flares that were not measured using the broad-band system.

\subsection{Analysing Correlations Between Production Parameters and Flares with}

\section{Apparent Liquid Carry-Over in North Dakota}

During the field measurements in North Dakota, posted information on site placards was recorded and used to search for relevant information about the sites in publicly accessible databases. Wells that were on the same site as a measured flare, or remote wells that were associated with the flare site, were identified using placard information and the GPS location, and central tank battery numbers were found in the North Dakota Industrial Commission Oil and Gas Division database (North Dakota Industrial Commision Oil and Gas Division 2021). This database contained completion dates and volumes of produced oil, produced water, produced gas, and total flared or vented gas for each well by month. Data for November 2019, the month of the field measurement campaign, were acquired and analyzed. Potential correlations between $\mathrm{Na}$ or $\mathrm{K}$ detection probability and available production parameters were explored. These included the mean or minimum well age of wells associated with the flare site (i.e., completion date subtracted from measurement date); total production volumes of oil, water, or gas; and total volume of flared/vented gas. 
A comparison of the ages of wells in the present sample and all wells listed in the North Dakota database is presented in Appendix B.

The well age and production volume data for flares with and without species detections were compared using non-parametric statistical hypothesis tests. Non-parametric hypothesis tests were used because the age and volume distributions did not appear to be normally distributed, which is an underlying assumption for parametric hypothesis tests. Although slight deviation from normality may be ignored if $n>30$, the sample size of flares without a successful detection is $<15$ and therefore a non-parametric test is preferred (Motulsky 2021).

The non-parametric Mann-Whitney test was used to compare the well age and production volume data of flares with and without detections. There are two interpretations of the Mann-Whitney test. The first interpretation assumes the shape and spread of the underlying sample populations are the same, such that their probability density functions differ in location only (Navidi 2010; Hart 2001). In this case, the p value generated by the Mann-Whitney test is used to determine whether the median of one population is statistically larger or smaller than the other (depending on the alternative hypothesis). The second interpretation is necessary when the shape or size of the underlying sample populations are not the same. For this latter case, the $\mathrm{p}$ value generated by the MannWhitney test determines whether one population tends to produce statistically larger values than the other (Motulsky 2021).

The well age and volumetric data for each flare were segregated into two groups for comparison: successful and null detections of $\mathrm{Na}$ and/or $\mathrm{K}$. The data for each parameter (e.g., total volume of produced oil, minimum well age, etc.) are presented as overlapping histograms with red or green indicating successful or null detections, respectively. Detailed results of the Mann-Whitney test are located in the top right corner of each plot, which includes the interpretation of the test as indicated by the null and alternative hypotheses. When the Mann-Whitney test results are to be interpreted as a difference in medians, the null and alternative hypotheses include the variable "Med" for median. 
Otherwise, the variable "Val" is used to represent the typical magnitude of a randomly selected value from the given population.

To the author's knowledge, there is no hypothesis test that compares the shape of two samples without also considering their location and/or spread. From visual inspection of the plots in coming sections, the small sample size of null detections $(n<15)$ means there is ambiguity in assessing whether or not the histograms of successful and null data have the same shape. Thus, Levene's test, a statistical hypothesis test for the difference in variance of two samples, was also considered. If Levene's test confirmed that the variances of histograms of successful or null detection were not statistically different, the MannWhitney test was assumed to be applicable when comparing population medians. Conversely, if Levene's test implied the variances were statistically different, then the Mann-Whitney test was used to indicate whether one population tended to have statistically larger values than the other. Finally, in addition to these hypothesis test, $95 \%$ confidence intervals for the difference in medians ( $\Delta$ median) were quantified using the bias-corrected and accelerated bootstrapping technique. The $\Delta$ median was calculated as the median value of interest (i.e., produced gas, oil, or water volume etc.) for successful detections minus the median value for null detections, and is included in each plot.

\subsubsection{Correlations of Species Detections with Well Age}

Histograms of mean and minimum well age of flares with successful and null species detections are plotted in Figure 5.5 (a) and (b), respectively. Well age data is calculated as the number of days since completion (DSC), equal to the date of the spectroscopic measurement minus the well completion. Levene's test on mean and minimum well age data showed, in both cases, the variance between data sorted by successful or null detection were not statistically different. Thus, the Mann-Whitney test was interpreted to compare population medians.

The well age is relevant because well production tends to decrease over its lifetime (Höök

et al. 2014; Bai et al. 2013). A higher volume production stream is associated with higher flow velocity and turbulence levels, which favour the entrainment of liquid aerosols in the gas phase of multiphase flow. These conditions could lead to poor liquid-gas 
disengagement during on-site separation processes, increasing the risk of liquid carry-over into flare systems. Moreover, an increased production volume of oil, water, or gas, or high volumes of flared gas could contribute to these unfavourable conditions for separations as further explored below.

In addition, wells that have been stimulated by hydraulic fracturing (which is common in North Dakota) tend to return fracturing fluid early in the production stage in addition to producing oil, water, and gas. Fracturing fluids may contain surfactants and other chemicals which, when present during the separation process, could increase risk of liquid carry-over via the production of foam (Bai et al. 2013; Shaban 1995). This is further reason for why flares associated with younger wells could be at a higher risk of liquid carry-over.

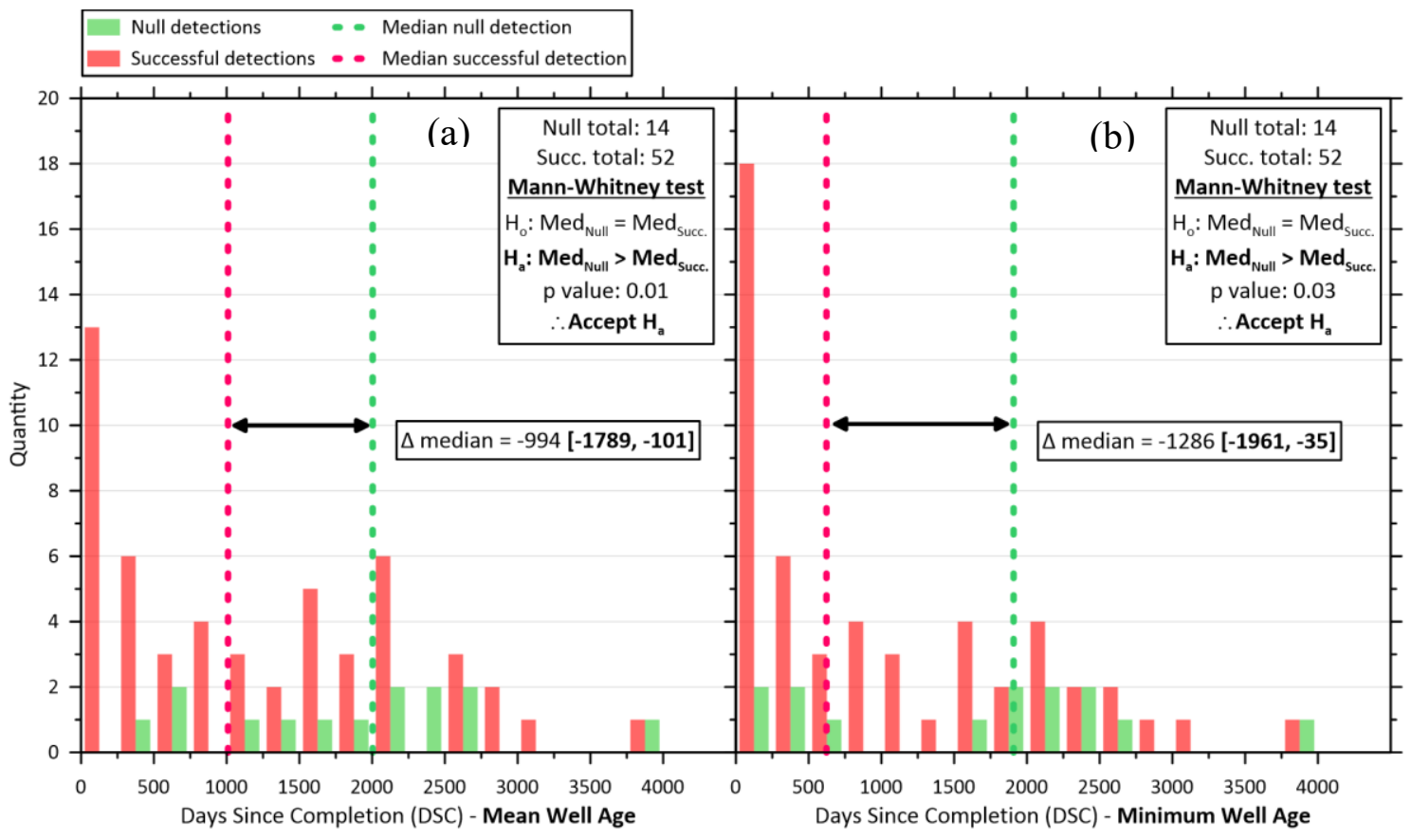

Figure 5.5: The (a) mean and (b) minimum well age of flares in North Dakota with successful and null species detections. In each case, the Mann-Whitney test is interpreted to compare population medians.

The result of the Mann-Whitney test in Figure 5.5 (a) and (b) reveal the median average well age and the median minimum well age are statistically lower for flares with species detections (the red dashed line) than without (the green dashed line). In both cases, the upper and lower bounds of the $95 \%$ confidence interval of $\Delta$ median are negative and nonzero, which supports the Mann-Whitney test results. The confidence interval for mean 
well age is more precise than minimum well age. It predicts that median average well age for flares with species detection is approximately three months less than flares without species detection. In comparison, the similar statistic for median minimum well age shows a difference of only one month.

Looking closely at Figure 5.5 (a), it is apparent that all 13 measured flares with mean well ages $<250$ DSC and 19 of 20 flares with mean well ages $<500$ DSC had positive detections. This further suggests that mean well age could be an important predictor of liquid carryover in North Dakota. Simply put, although species detections were seen in flares of all ages, the likelihood of species detections was statistically associated with younger wells.

\subsubsection{Correlations of Species Detections with Produced Water or Oil Volumes}

Histograms of total volume of produced oil and water for flares with and without species detections are plotted in Figure 5.6 (a) and (b), respectively. The volumetric data for each flare (whether it be for produced oil, water, gas, or flared/vented gas) is the sum of its matched well's reported volumes for the month of November, 2019. For both the produced oil and water data sets, Levene's test showed that the variance between data sorted by successful or null detection was not statistically different. Thus, the Mann-Whitney test was interpreted to compare population medians.

The Mann-Whitney tests in Figure 5.6 suggest that both the median total volume of produced oil and produced water are statistically larger for flares with species detections than without. In both cases, the upper and lower bounds of the $95 \%$ confidence interval of $\Delta$ median are positive and nonzero, which supports the Mann-Whitney test results. The confidence interval on the $\Delta$ median for produced oil is more precise than that for produced water, and in both cases the lower bound is $>2510^{3} \mathrm{ft}^{3} \mathrm{mo}^{-1}$. For context, the median oil production is $8610^{3} \mathrm{ft}^{3} \mathrm{mo}^{-1}$ for all sites in Figure 5.6 (a), which is equivalent in volume to $\sim 1$ Olympic size swimming pool per month. The analysis suggests there is a $>95 \%$ chance the difference in median oil or water production could have filled $\sim 1 / 4$ of an Olympic size swimming pool in November, 2019. 


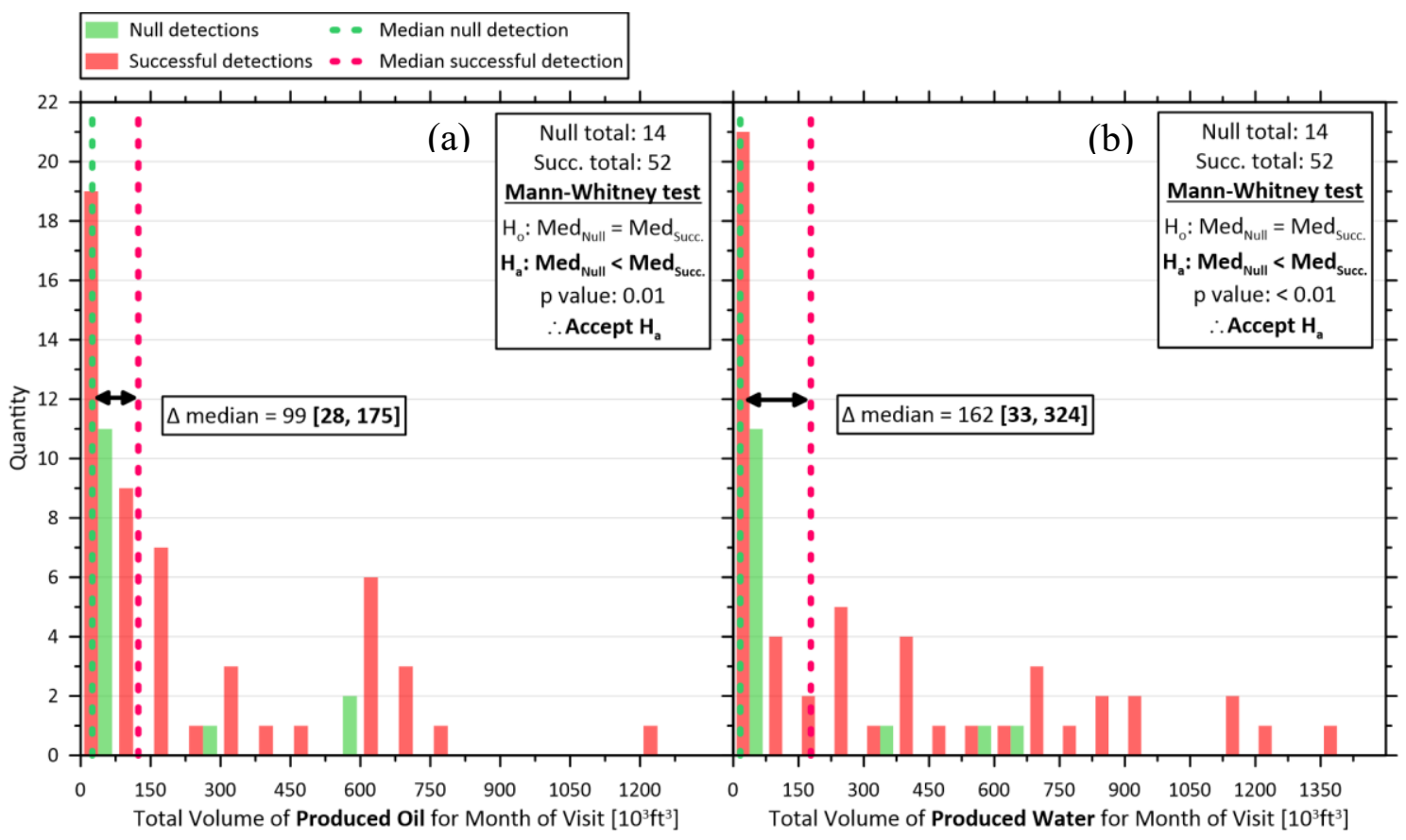

Figure 5.6: The total reported volume of (a) oil and (b) water produced by wells associated with flares with and without species detections, during the month of November, 2019. In each case, the Mann-Whitney test is interpreted to compare population medians.

Of flares with evidence of liquid carry-over, Figure 5.6 (a) and (b) reveal that $92 \%$ (33 of 36) had produced oil volumes $>7510^{3} \mathrm{ft}^{3} \mathrm{mo}^{-1}$ and $91 \%$ (30 of 33) had produced water volume $>7510^{3} \mathrm{ft}^{3} \mathrm{mo}^{-1}$. However, below this arbitrary threshold, the likelihood of apparent liquid carry-over is lower with 63 to $65 \%$ exhibiting detectable $\mathrm{Na}$ or $\mathrm{K}$ signatures. This suggests both that higher total production volumes of oil and water may be practically and statistically significant predictors of liquid carry-over in North Dakota, and that other factors are also likely relevant since the likelihood in all cases is still high.

\subsubsection{Correlations of Species Detections with Produced Gas or Flared / Vented}

\section{Volumes}

Histograms of the total volume produced gas and flared/vented gas for flares with and without species detections are shown in Figure 5.7. Levene's test shows the variances of produced gas volume data sorted by successful or null detection were not statistically different. However, there was a statistically significant difference in the variances of flare/vent gas volume data sorted by successful or null detections. This means the MannWhitney test should be interpreted as comparing population medians for produced gas data, 
and as the tendency for one population to produce larger values than the other for flared/vented gas data.

The total number of flares included in flare/vent gas data (Figure 5.7 (b)) is reduced by four when compared to all other production parameters (two successful and two null detections). These flares are removed because their wells did not report any flared/vented gas volumes for the month of November 2019 even though an operating flare was observed at the time of measurement.

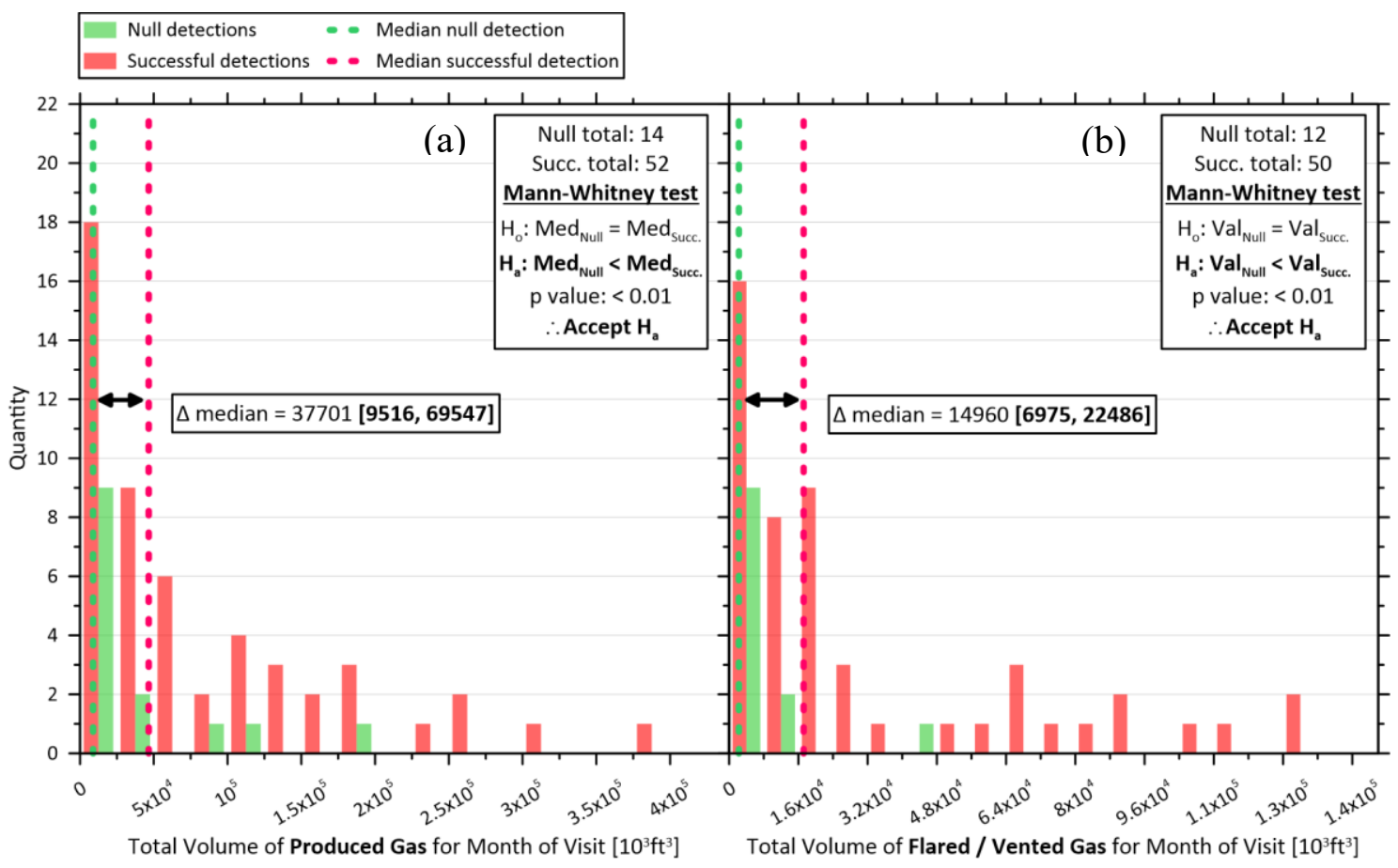

Figure 5.7: The total reported volume of gas that was (a) produced and (b) flared/vented by wells associated with flares with and without species detections, during the month of November 2019. The Mann-Whitney test compares population medians for produced gas volumes, and the tendency for one population to produce larger values than the other for flared/vented gas volumes.

The result of the Mann-Whitney test in Figure 5.7 (a) show the median total volume of produced gas is statistically larger for flares with species detections than without. Whereas in Figure 5.7 (b), the test shows that flared/vented gas volume had the tendency to be statistically larger for flares with species detections than without. In both cases, the upper and lower bounds of the $95 \%$ confidence interval of $\Delta$ median are positive and nonzero, which supports the Mann-Whitney test results. The confidence interval for flared/vented 
gas is $\sim 3 \times$ more precise than that of produced gas, and in both cases, the lower bound is $\gtrsim 700010^{3} \mathrm{ft}^{3} \mathrm{mo}^{-1}$.

In particular, the confidence intervals in Figure 5.7 (a) suggest there is $>95 \%$ chance that flares with evidence of liquid carry-over produce $950010^{3} \mathrm{ft}^{3} \mathrm{mo}^{-1}$ more gas than flares without evidence. For perspective, suppose the internal diameter of pipe transporting gas to the separation tanks or through flare systems at UOG facilities in North Dakota is 6 in. To transport an additional $950010^{3} \mathrm{ft}^{3} \mathrm{mo}^{-1}$ of gas per month through a 6 in diameter pipe requires an increase in the average flow velocity of $\sim 18.7 \mathrm{ft} \mathrm{s}^{-1}$, or $\sim 5.7 \mathrm{~m} \mathrm{~s}^{-1}$. Thus, this

crude estimation suggests that average gas velocities could be $\sim 19 \mathrm{ft} \mathrm{s}^{-1}$, or $\sim 6 \mathrm{~m} \mathrm{~s}^{-1}$ greater for flares with liquid carry-over. Again assuming 6" diameter piping, this compares to a median gas velocity for all sites in Figure 5.7 (a) of $\sim 75 \mathrm{ft} \mathrm{s}^{-1}$, or $\sim 23 \mathrm{~m} \mathrm{~s}^{-1}$.

The results of Figure 5.7 suggest there may be a threshold of produced gas or flared/vented gas volume above which the likelihood of liquid carry-over is greatly increased. Figure 5.7 (a) shows that $71 \%$ of flares below, versus $90 \%$ of flare above an arbitrary produced gas volume threshold of 50,000 $10^{3} \mathrm{ft}^{3} \mathrm{mo}^{-1}$ had evidence of liquid carry-over. Similarly, for arbitrary flared/vented gas volume threshold of $16,00010^{3} \mathrm{ft}^{3} \mathrm{mo}^{-1}, 69 \%$ of flares with lower volumes versus $96 \%$ with higher volumes had evidence of liquid carry-over. These results are analogous to those of the produced liquids analysis in Section 5.3.2, and suggest that produced and flared/vented volume of gas is also practically and statistically significant indicators of liquid carry-over in North Dakota. An attempt to elucidate the relative importance of different correlating parameters with liquid carry-over is presented in the next section.

\subsection{Relative Importance of Production Parameters in Predicting Liquid Carry-Over in North Dakota Flares}

The relative accuracy and preciseness with which well age and volumetric data predict liquid carry-over (as evidenced by the detection of $\mathrm{Na}$ or $\mathrm{K}$ species) in North Dakota flares was investigated using logistic regression. Identifying which production parameters are strong predictors of species detection could elucidate causes and help establish a basis for future actions or recommendations to reduce the likelihood of liquid carry-over. 


\subsubsection{Logistic Regression Model for Predicting Species Detection}

In a logistic regression model, predictor variable(s) are typically assumed to have a linear relation with the response variable (Menard 2002). In this case, the response variable is the binary "yes / no" classification of species detection. Alternatively stated, the model predicts whether a flare will have evidence of liquid carry-over or not. The functional form

of the linear logistic regression model is, $y=\beta_{o}+\beta_{1} x_{1}+\cdots+\beta_{n} x_{n}$, where each predictor variable $(x)$ is multiplied by its scalar coefficient $(\beta)$; these coefficients are estimated through logistic regression. Since the response variable is binary, ordinary least squares regression techniques are of no use; logistic regression models require maximum likelihood techniques to estimate coefficients. For this study, the logistic regression models were generated using the FITGLM function in MathWorks ${ }^{\circledR}$ (2021). For more details on logistic regression models see Menard (2002), Martinez and Martinez (2007), or Allen (1997).

\subsubsection{Assessing the Correlations Among Predictor Variables}

In theory, the change in the probability, odds, or log-odds of the species detection status due to a unit-change in each production parameter is one way to compare their relative strength as predictors through a multivariable logistic regression model. However, the estimated values of the coefficients $\beta$ in the linear logistic regression model are strongly influenced by correlation among predictor variables (Menard 2002; Allen 1997; Belsley et al. 1980). Correlation (or collinearity - its equivalent term for regression problems) can lead to inaccurate or imprecise coefficient estimates (Belsley et al. 1980). Thus, it is necessary to identify correlations between predictor variables before generating any multivariable logistic regression model.

The degree of correlation among variables is commonly assessed via Pearson's correlation coefficient (Midi et al. 2010). A correlation matrix of Pearson's correlation coefficient was generated for all six predictor variables listed in the opening paragraph of Section 5.3, and are shown bolded in Table 5.2. The associated 95\% confidence interval for each correlation coefficient is presented square brackets. A statistical hypothesis test (the t test) was performed on each correlation coefficient to determine whether it is statically different than 
zero, with $\alpha=0.05$. The results from the hypothesis test showed that every correlation coefficient included in Table 5.2 was statistically significant (i.e., $\neq 0$ ).

Table 5.2: Pearson correlation coefficient matrix for predictor variables: produced oil, water, and gas; flared/vented gas; and mean well age, and minimum well age.

\begin{tabular}{|c|c|c|c|c|c|}
\hline $\begin{array}{c}\text { Produced } \\
\text { Oil }\end{array}$ & $\begin{array}{c}\text { Produced } \\
\text { Water }\end{array}$ & $\begin{array}{c}\text { Produced } \\
\text { Gas }\end{array}$ & $\begin{array}{c}\text { Flared/ } \\
\text { Vented Gas }\end{array}$ & $\begin{array}{l}\text { Minimum } \\
\text { Well Age }\end{array}$ & $\begin{array}{l}\text { Mean Well } \\
\text { Age }\end{array}$ \\
\hline \multirow[t]{6}{*}{1} & $\begin{array}{c}0.85 \\
{[0.77,0.91]}\end{array}$ & $\begin{array}{c}\mathbf{0 . 8 7} \\
{[0.79,0.92]}\end{array}$ & $\begin{array}{c}\mathbf{0 . 6 9} \\
{[0.53,0.8]}\end{array}$ & $\begin{array}{c}\mathbf{- 0 . 6 8} \\
{[-0.79,-0.51]}\end{array}$ & $\begin{array}{c}\mathbf{- 0 . 7 3} \\
{[-0.83,-0.59]}\end{array}$ \\
\hline & 1 & $\begin{array}{c}\mathbf{0 . 7 1} \\
{[0.56,0.81]}\end{array}$ & $\begin{array}{c}\mathbf{0 . 6 8} \\
{[0.51,0.79]}\end{array}$ & $\begin{array}{c}-\mathbf{0 . 7 1} \\
{[-0.82,-0.56]}\end{array}$ & $\begin{array}{c}-\mathbf{0 . 7 7} \\
{[-0.86,-0.65]}\end{array}$ \\
\hline & & 1 & $\begin{array}{c}\mathbf{0 . 8} \\
{[0.69,0.87]}\end{array}$ & $\begin{array}{c}-\mathbf{0 . 6 6} \\
{[-0.78,-0.49]}\end{array}$ & $\begin{array}{c}-\mathbf{0 . 6 8} \\
{[-0.8,-0.52]}\end{array}$ \\
\hline & & & 1 & $\begin{array}{c}-\mathbf{0 . 5 9} \\
{[-0.73,-0.39]}\end{array}$ & $\begin{array}{c}\mathbf{- 0 . 6 1} \\
{[-0.75,-0.42]}\end{array}$ \\
\hline & & & & 1 & $\begin{array}{c}\mathbf{0 . 9 5} \\
{[0.93,0.97]}\end{array}$ \\
\hline & & & & & 1 \\
\hline
\end{tabular}

Produced oil, water, gas, and flared/vented gas volumes have moderate-to-high positive correlations with one another, and similarly strong negative correlations with the mean and minimum well age data. The negative correlation between well age and production data is expected; younger wells typically have higher production than older wells. Similarly, positive correlations among volumetric parameters suggests that the produced volumes of oil, gas, or water returning to the surface are linked as expected. In addition, the mean and minimum well age data have strong positive correlations with one another as expected. The strength of correlation among predictor variables suggests collinearity could be a problem when interpreting the estimated coefficients of a multivariable logistic regression model.

The collinearity among predictor variables can also be mathematically assessed through variance inflation factors (VIF) (Midi et al. 2010). The VIF quantifies the relative increase in a coefficient's variance due to collinearity between its predictor variable and other predictor variables in a multivariable model (Midi et al. 2010). The VIF $=1$ if a predictor variable does not experience collinearity with any other predictor variables. The VIF for a multivariable model containing all six predictor variables and is shown in Table 5.3. 
Table 5.3: The variance inflation factor (VIF) used to model the strength of collinearity among the six production parameters in a multivariable model that contains all six predictor variables (these include produced oil, water, and gas; flared/vented gas; and mean well age and minimum well age).

\begin{tabular}{|c|c|c|c|c|c|c|}
\cline { 2 - 6 } \multicolumn{1}{c|}{} & $\begin{array}{c}\text { Produced } \\
\text { Oil }\end{array}$ & $\begin{array}{c}\text { Produced } \\
\text { Water }\end{array}$ & $\begin{array}{c}\text { Produced } \\
\text { Gas }\end{array}$ & $\begin{array}{c}\text { Flared/ } \\
\text { Vented } \\
\text { Gas }\end{array}$ & $\begin{array}{c}\text { Minimum } \\
\text { Well Age }\end{array}$ & $\begin{array}{c}\text { Mean } \\
\text { Well Age }\end{array}$ \\
\hline $\begin{array}{c}\text { VIF } \\
\text { (relative increase in } \sigma^{2} \text { ) }\end{array}$ & 8.42 & 5.36 & 7 & 3.24 & 11.68 & 14.25 \\
\hline
\end{tabular}

Unfortunately, there is no consensus in the literature on the magnitude of VIFs that indicates a collinearity problem. Some commonly used VIF thresholds which are said to indicate collinearity, or are cause for concern are: 2.5, 5, 10 (Johnston et al. 2018; Menard 2002). Regardless of which threshold is used, Table 5.3 shows the variance of each production parameter coefficient in the six-variable model increases by $>3 \times$ due to collinearity. According to Midi et al. (2010), the square root of VIF is equal to the relative increase in the standard error (i.e., the standard deviation) which, for all but one production parameter in the six-variable model (flared/vented gas), increases by $>2 \times$. These findings imply the six-variable model should not be used to compare the influence of individual production parameters on the probability of finding evidence of liquid carry-over, due to the negative effect collinearity has on the preciseness and accuracy of model coefficients.

An alternative technique for comparing production parameters involves generating six logistic regression models (one for each production parameter). Then, the ability of each model to classify the species detection status can be compared using their "true positive detection rate", which is discussed in detail in the next section. This comparison of classifier ability can then be extended to compare single-variable and multi-variable models, or in other words, to compare the models of every possible combination of the six production parameters.

\subsubsection{Comparing the Precision and Accuracy of Logistic Regression Models when Classifying the Species Detection Status of North Dakota Flares}

For a logistic regression model to be useful in the present analysis, it must accurately and precisely classify flares with evidence of liquid carry-over. This ability can be described as the "true positive detection rate", which is equal to the number of true positive detections that are classified as such, divided by the total number of true positive detections (Majnik 
and Bosnić 2013). It is arguably equally or more important to consider the "false positive detection rate" of a classifier. The false positive detection rate is equal to the number of negative detections that were classified as positive detections by a classifier, divided by the total number of true negative detections (Majnik and Bosnić 2013). A perfect classifier would have a true positive and false positive detection rate of 1 and 0 , respectively.

The false positive detection rate of a classifier is analogous to the $\alpha$ (i.e., the type 1 error) of a statistical hypothesis test in that it describes the percent of detections that were wrongly classified as true. This analysis compares the true positive detection rates for each model at the false positive detection rate of $5 \%$. Using the false positive detection rate of $5 \%$ here is equivalent to using the value of $\alpha=0.05$ for statistical hypothesis tests. True positive and false positive detection rates belong to the receiver operating characteristic (ROC) curve of each model, which was generated using the same data as used in regression to estimate model coefficients. For detailed information regarding ROC curves see Majnik and Bosnić (2013).

In Figure 5.8 and Figure 5.9, the "true" true positive detection rate (at 5\%) is estimated for each model. Alternatively stated, the "true" true positive detection rate is true positive detection rate of each model if it were used to classify the entire flare population in North Dakota (Macskassy et al. 2005). Estimates were generated using bias-corrected accelerated bootstrapping and vertical averaging techniques. In brief, bootstrapping involved resampling production parameter data (with replacement) 50,000 times for each model. The mean true positive detection rate from the resamples is indicated by the circle symbol and the $95 \%$ confidence interval is indicated by the whiskers. For details on how vertical averaging was used to obtain a true positive detection rate at a 5\% false positive rate, see Fawcett (2004, 2006), Macskassy et al. (2005), or Provost et al. (1997). Furthermore, for more information on, and examples of how, true positive detection rates were calculated for this study, see documentation on the PERFCURV function in MathWorks ${ }^{\circledR}$ (2021). 


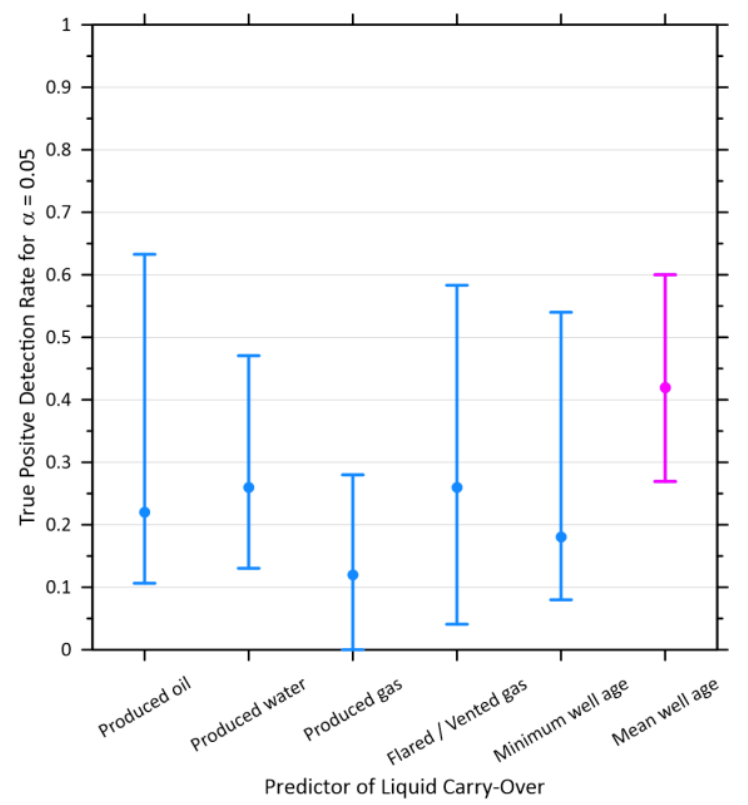

Figure 5.8: The true positive detection rate at $\alpha=0.05$ for six unique single-variable logistic regression models. A model was generated for each of the following production parameters: produced oil, water, and gas; flared/vented gas; and mean well age and minimum well age.

All of the confidence intervals in Figure 5.8 are all fairly imprecise, considering they span at least $25 \%$ of all possible the true positive detection rates. This makes it difficult to interpret the accuracy of each model (a perfectly accurate model has a true positive detection rate equal to one). However, Figure 5.8 suggest that out of all six production parameters, mean well age is likely the most accurate classifier of species detection status. This result is based on its relatively narrow confidence interval (spanning $\sim 33 \%$ of all possible true positive detection rates), which includes some of the highest true positive detection rates of any single-variable model shown (the upper bound of mean well age is $60 \%$ whereas that of produced oil is $\sim 65 \%$ ). More importantly though, Figure 5.8 shows that no single-variable model is on its own an effective classifier of species detection status. Simply put, the highest accuracy expected from most single-variable models is $60 \%( \pm 5 \%)$, which misclassifies $\sim 40 \%( \pm 5 \%)$ of flares that contain evidence of liquid carry-over.

A subsequent attempt was made to determine whether a model generated from any combination of the six production parameters is an adequate classifier of species detection status. In this approach, the effects of collinearity were ignored and only true positive detection rates were considered with the goal of identifying the model that classifies species detection status of North Dakota flares with the highest precision and accuracy. There are 
63 unique models that can be generated from the six production parameters, and their "true" true positive detection rates with $95 \%$ confidence intervals are shown in Figure 5.9. The models in Figure 5.9 progress from single-variable models on the left, through 2-, 3-, 4-, and 5-variable models, to the six-variable model on the right (the six-variable model is model number 63). The blue and purple colour models in in Figure 5.9 are the singlevariable models shown in Figure 5.8.

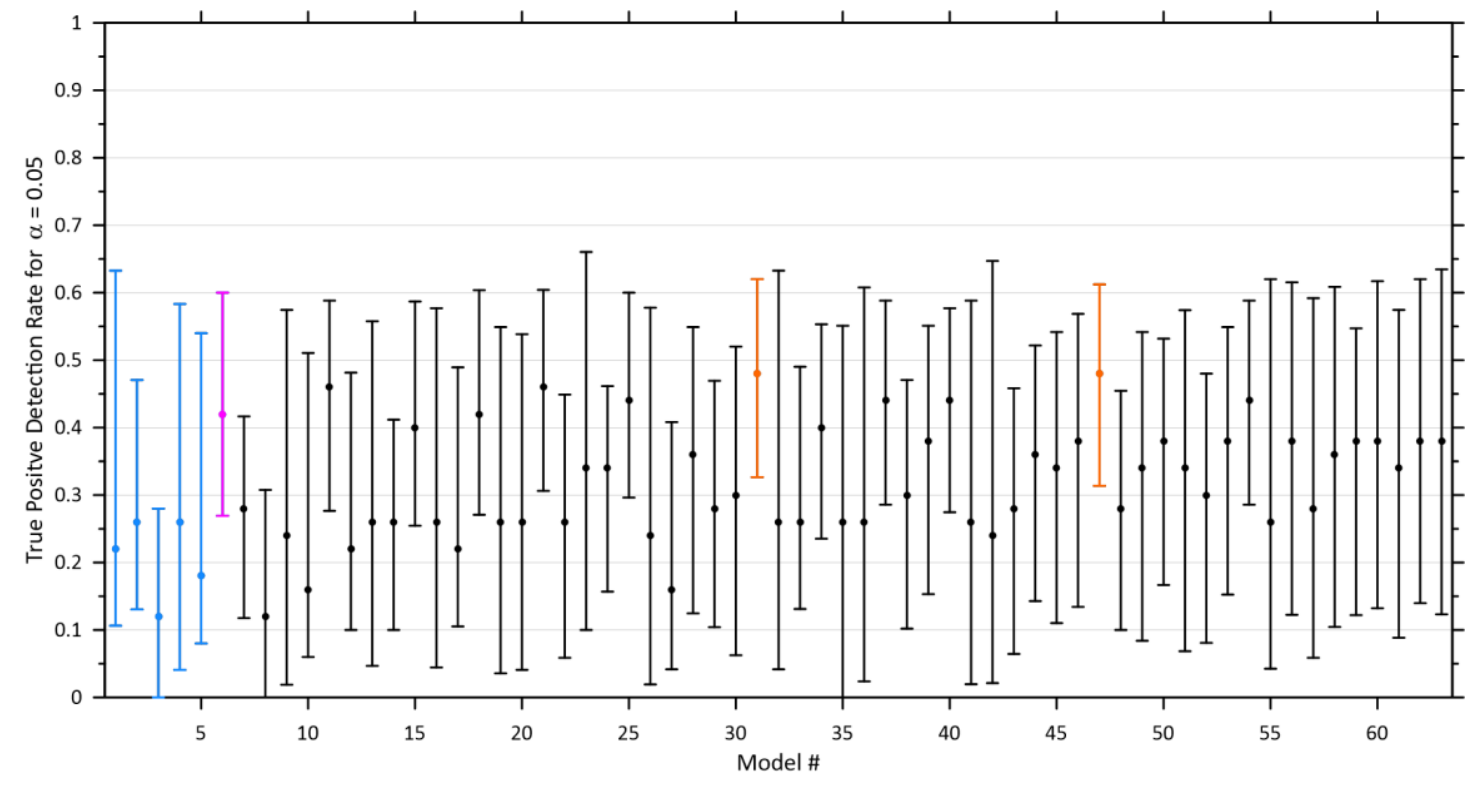

Figure 5.9: The true positive detection rate of models that were generated from every possible combination of the six production parameters.

Figure 5.9 shows that no model is expected to have an accuracy $>70 \%$, meaning at best, $30 \%$ of flares with evidence of liquid carry-over will be misclassified. There are, however, some models with high precision estimates of true positive detection rate that are expected to be as accurate or slightly more accurate than the purple model (two of these models are highlighted in orange). All of these models, including the two drawn in orange, consider the production parameter mean well age. In addition, the left and right orange coloured models consider produced oil and minimum well age; and produced oil, produced water, and minimum well age, respectively. The increased complexity of the orange models (due to the extra production parameters) does not appear to raise the accuracy or precision of their estimated true positive detection rate by any reasonable amount when compared to the purple model. This implies the additional parameters in the orange model might not be 
as important as mean well age when classifying the species detection status of North Dakota flares.

\subsubsection{Summarizing the Results from the Correlation and Classifier Analyses}

The important correlations between species detections and the production parameters for well age and production and flaring/venting volumes are summarised Table 5.4. In short, flares containing evidence of liquid carry-over are statistically associated with higher production volumes, higher flared/vented gas volumes, and younger wells. A correlation matrix showed that volumetric data of produced oil, water, gas, and flared/vented gas have moderate-to-high positive correlations with one another, and similarly strong negative correlations with the mean and minimum well age data. In addition, the mean and minimum well age data have strong positive correlations with one another.

Table 5.4: A summary of the correlations between species detections and the production parameters for well age, production volumes, and flared gas volumes.

\begin{tabular}{|c|c|}
\hline Type of Production Parameter & Result of Hypotheses Tests \\
\hline Production volume & $\begin{array}{c}\text { The median mean and minimum well age are statistically } \\
\text { lower for flares with species detection than without }\end{array}$ \\
\hline Flaring / Venting gas volume & $\begin{array}{c}\text { The median volume of produced oil, water, gas are } \\
\text { statistically higher for flares with species detection than } \\
\text { without }\end{array}$ \\
\hline $\begin{array}{c}\text { The volume of flared / vented gas tends to be statistically } \\
\text { higher for flares with species detection than without }\end{array}$ \\
\hline
\end{tabular}

The classifier analyses comparing every possible logistic regression model that could be generated from linear combinations of the six production parameters showed that no model was an accurate or precise classifier of liquid carry-over. However, the single- and multivariable models containing mean well age had the most precise true positive detection rate estimates. The marginal increase in preciseness and accuracy of the multi-variable models containing mean well age over its single-variable model suggests the linear combinations of additional production parameters do not significantly improve the true positive detection rate estimate. Thus, although a model on well age alone only is only estimated to net a true positive detection rate of $60 \%$ (at best), this is the best classifier of species detection status out of the six production parameters considered in this study. 
The generally poor performance of these classifiers may indicate that an underlying model to predict liquid carry-over is more complex than the simple linear combinations investigated here, or more likely, that other factors are also important to consider. Some factors that might contribute to liquid carry-over are the design and sizing of the separator system, the upkeep and operational practices of the separator (e.g., frequency of emptying or dump-valve actuation to drain out liquids, damage or maintenance standard of equipment, etc.) or even weather at the time of measurement since the separation process may be sensitive to temperature and downstream pressure. Further investigation of these factors could be interesting to pursue, but regardless, these field experiments and analyses reveal that liquid carry-over into flare systems appears to be a common occurrence for flares in North Dakota, South Saskatchewan, and Ecuador. 


\section{Chapter 6 Conclusion}

A spectrometer system was developed to measure the spectral emission of flare flames from the boundaries of upstream oil and gas (UOG) facilities. Two versions of the spectrometer system were built, one capable of measuring sodium $(\mathrm{Na})$ atomic emission at 588.99 / $589.59 \mathrm{~nm}$; and the other capable of measuring potassium (K) atomic emission at 766.48 / $769.89 \mathrm{~nm}$ and broadband soot emission from $~ 500$ to $900 \mathrm{~nm}$ (which was used to calculate flame temperature). These two species were targeted because they are commonly found in water samples from oil and gas formations and, given their absence in natural gas, their presence in flare flames could be concluded as proof of carry-over produced water into flare systems. Moreover, the low boiling temperatures $(<2300 \mathrm{~K})$ of common salts such as sodium chloride $(\mathrm{NaCl})$ and potassium chloride $(\mathrm{KCl})$ meant that these species were most likely to reach the flare flame, become excited, and produce detectable emission signatures.

The spectrometer systems were deployed in two different field measurement campaigns to determine, for the first time ever, the frequency in which liquid carry-over occurs under field conditions at operational UOG facilities. The first measurement campaign targeted eighty-one flares in the Bakken region (i.e., the Williston basin) of North Dakota (76) and southern Saskatchewan (5) in November, 2019; the second campaign measured 14 flares in the Amazon basin region of Ecuador in February, 2020. Potential emission signatures of $\mathrm{Na}$ or $\mathrm{K}$ were subjected to three peak identification metrics whose purpose was to classify "successful" and "null" detections. To be classified as a successful detection, a potential emission signature had to have a signal-to-noise ratio greater than $3 \sigma$ of the background, have an integrated intensity ratio greater than 1.22 for $\mathrm{Na}$ emission and 0.9 for $\mathrm{K}$ emission, and pass an f-test $(\alpha=0.01)$ comparing the fit of an atomic emission model to that of a straight-line background model. Flares with at least one successful detection of $\mathrm{Na}$ or $\mathrm{K}$ were considered to have evidence of liquid carry-over at the time of measurement.

Using these criteria, $72 \%$ of all 95 measured flares in this study $\left(95^{\text {th }}\right.$ percentile confidence interval of 62 to $80 \%$ ) had detectable $\mathrm{Na}$ or $\mathrm{K}$ emission signatures indicating carry-over of 
produced water aerosols into flare flames. More specifically, $77 \%$ (67 to $85 \%$ ) of flares in the Bakken region of the Williston basin (including flares in southern Saskatchewan and North Dakota), and 43\% (21 to 71\%) of flares in the Amazon basin of Ecuador had detectable evidence of liquid carry-over. Notably, these results suggest that liquid carryover into flare systems is common at UOG production sites.

Potential indicators of liquid carry-over in North Dakota were compared using publicly accessible data for well age, production volumes, and flared / vented gas volumes. The results from Levene's test and the Mann-Whitney test showed that flares containing evidence of liquid carry-over were statistically associated with larger production volumes and flare /vented gas volumes, and younger wells. Using logistic regression techniques, attempts were made to determine the relative importance of these parameters and whether they might be used to predict liquid carry-over. Although all unique combinations of six potential parameters (total production volume of oil, water, gas; total volume of flared/vented gas; and mean and minimum well age) were considered, no model was estimated to predict with a true positive detection rate greater than $66 \%$. This generally poor performance suggests that the drivers of liquid carry-over are likely more complex than the considered simple linear relationships of production parameters and that other factors are likely important such as the design, sizing, or operation of on-site separation equipment.

Most importantly, the present results indicate that the carry-over of liquid into flare systems appears to be a frequent occurrence at upstream production sites and has now been observed at operational facilities in two continents (North and South America), three countries (Canada, U.S.A, and Ecuador) and as many or more jurisdictions. Given ongoing research highlighting adverse impacts of entrained salts on black carbon, CO, and VOC emissions from simulated flare flames (e.g., Roth et al. 2020), these results have profound implications for our understanding of the potential impacts of flare emissions globally.

\subsection{Future Considerations}

Although the present system was able to detect the presence of $\mathrm{Na}$ and $\mathrm{K}$ species in flare flames, it could not quantify their concentrations. Understanding the likely range of salt 
loading rates in flare flames under field conditions is critical to guiding lab-based experiments and bounding potential effects of increased pollutant emissions. Unfortunately at present, there is no known practical way to estimate salt loading rates from the field data of flare flames. It may be possible to match the intensities of emission signatures in field data to those obtained using a lab-scale flare flame with a known salt loading rate under similar measurement conditions. Exploratory sensitivity tests conduced at the Carleton University Flare Facility (CUFF) were discussed in Chapter 3, but due to the limited lab space, typical stand-off distances could not be tested. Future experiments should involve various salt loading rates and stand-off distances, and could possibly be used to generate a catalog of test conditions that could be matched with setup conditions in the field in an effort to estimate likely bounds on salt loading rates.

Moreover, to determine whether the detection rates of liquid carry-over are in fact representative of the global UOG industry, additional flare measurements should be performed in more oil producing regions. For example, augmented emissions from the Permian basin in Texas is important because it is one of the largest oil producing regions in North America. Similarly experiments in the West Siberian basin in Russia could be especially insightful given the suspected importance of Russian flare emissions on radiative forcing in the artic. 


\section{References}

Alkemade, C.T.J., Bleekrode, R., Burger, J.C., Butler, C.C., Cath, P.G., Fassel, V.A., Gilbert, P.T., Gillies, W., Herrmann, R., Kniseley, R.N., Mavrodineanu, R., Menis, O., Müller-Herget, W., Pinta, M., Rains, T.C., Smith, R., Willis, J.B., Winefordner, J.D., and Yamasaki, G.K. (1970). Analytical Flame Spectroscopy, Analytical Chemistry. Macmillan Education UK, London.

Alkemade, C.T.J., Hollander, T., Snelleman, W., and Zeegers, P.J.T. (1982). Metal Vapours in Flames. Pergamon Press.

Allen, M.A. (1997). Understanding Regression Analysis. Plenum Press, New York and London.

Altarawneh, M., Dlugogorski, B.Z., Kennedy, E.M., and Mackie, J.C. (2009). Mechanisms for formation, chlorination, dechlorination and destruction of polychlorinated dibenzo-p-dioxins and dibenzofurans (PCDD/Fs). Prog. Energy Combust. Sci., 35(3):245-274.

API (2020). API standards: International usage and Deployment. American Petroleum Institute (API).

API (2014). API Standard 521: Pressure-relieving and Depressuring Systems. American Petroleum Institute (API).

Bai, B., Goodwin, S., and Carlson, K. (2013). Modeling of frac flowback and produced water volume from Wattenberg oil and gas field. J. Pet. Sci. Eng., 108:383-392.

Belsley, D.A., Kuh, E., and Welsch, R.E. (1980). Regression Diagnostics — Identifying Influential Data and Sources of Collinearity. John Wiley \& Sons.

Blondes, M.S., Gans, K.D., Engle, M.A., Kharaka, Y.K., Reidy, M.E., Saraswathula, V., Thordsen, J.J., Rowan, E.L., and Morrissey, E.A. (2018). U.S. Geological Survey National Produced Waters Geochemical Database (ver. 2.3, January 2018).

Burtt, D., Corbin, D.J., Kopp, G., and Johnson, M.R. (2020). Investigation of Methane Emissions from Flares in a Turbulent Crosswind., in AGU Fall Meeting, San Francisco, CA.

Carinhana, D., Barreta, L.G., Rocha, C.J., Dos Santos, A.M., and Bertran, C.A. (2008). Determination of liquefied petroleum flame temperatures using emission spectroscopy. J. Braz. Chem. Soc., 19(7):1326-1335.

Chandra Sekharan, K. (2018). On the use of C2* emission spectroscopy for thermometry of flames in propulsion systems. Delft University of Technology.

Cleveland, W.S. and Devlin, S.J. (1988). Locally Weighted Regression : An Approach to Regression Analysis by Local Fitting. J. Am. Stat. Assoc., 83(403):596-610. 
Conrad, B.M. and Johnson, M.R. (2017). Field measurements of black carbon yields from gas flaring. Environ. Sci. Technol., 51(3):1893-1900.

Conrad, B.M., Thornock, J.N., and Johnson, M.R. (2020). The effect of multiple scattering on optical measurement of soot emissions in atmospheric plumes. $J$. Quant. Spectrosc. Radiat. Transf., 254:107220.

Cushing, L.J., Chau, K., Franklin, M., and Johnston, J.E. (2021). Up in smoke: Characterizing the population exposed to flaring from unconventional oil and gas development in the contiguous US. Environ. Res. Lett., 16(3).

Dekking, F.M., Kraaikamp, C., Lopuhaä, H.P., and Meester, L.E. (2005). A Modern Introduction to Probability and Statistics, Journal of Chemical Information and Modeling, Springer Texts in Statistics. Springer London, London.

Delichatsios, M.A., Orloff, L., Mutual, F., and Approach, E. (1984). Entrainment measurements in turbulent buoyant jet. Symp. A Q. J. Mod. Foreign Lit., .

Ding, S., Wang, J., and Xu, X. (2016). Polarimetric remote sensing in oxygen A and B bands: Sensitivity study and information content analysis for vertical profile of aerosols. Atmos. Meas. Tech., 9(5):2077-2092.

Efron, B. and Tibshirani, R.J. (1994). An Introduction to the Bootstrap, An Introduction to the Bootstrap. Springer Science and Business Media Dordrecht.

Fawcett, T. (2006). An introduction to ROC analysis. Pattern Recognit. Lett., 27(8):861874.

Fawcett, T. (2004). ROC graphs: Notes and practical considerations for researchers. Mach. Learn., 31(1):1-38.

Gaydon, A.G. (1974). The Spectroscopy of Flames, Endeavour. Springer Netherlands, Dordrecht.

Gibson, J.H., Grossman, W.E.L., and Cooke, W.D. (1963). Excitation Processes in Flame Spectrometry. Anal. Chem., 35(3):266-277.

Goldenstein, C.S., Miller, V.A., Mitchell Spearrin, R., and Strand, C.L. (2017).

SpectraPlot.com: Integrated spectroscopic modeling of atomic and molecular gases. J. Quant. Spectrosc. Radiat. Transf., 200:249-257.

Goldstein, S.A. and Walters, J.P. (1976). A review of considerations for high-fidelity imaging of laboratory spectroscopic sources-Part I. Spectrochim. Acta Part B At. Spectrosc., 31(4):201-220.

Goody, R.M. and Yung, Y.. (1995). Atmospheric Radiation: Theoretical Basis, 2nd ed, Quarterly Journal of the Royal Meteorological Society. Oxford University Press. 
Gordon, I.E., Rothman, L.S., Hill, C., Kochanov, R.V., Tan, Y., Bernath, P.F., Birk, M., Boudon, V., Campargue, A., Chance, K.V., Drouin, B.J., Flaud, J.-M., Gamache, R.R., Hodges, J.T., Jacquemart, D., Perevalov, V.I., Perrin, A., Shine, K.P., Smith, M.-A.H., Tennyson, J., Toon, G.C., Tran, H., Tyuterev, V.G., Barbe, A., Császár, A.G., Devi, V.M., Furtenbacher, T., Harrison, J.J., Hartmann, J.-M., Jolly, A., Johnson, T.J., Karman, T., Kleiner, I., Kyuberis, A.A., Loos, J., Lyulin, O.M., Massie, S.T., Mikhailenko, S.N., Moazzen-Ahmadi, N., Müller, H.S.P., Naumenko, O.V., Nikitin, A.V., Polyansky, O.L., Rey, M., Rotger, M., Sharpe, S.W., Sung, K., Starikova, E., Tashkun, S.A., Auwera, J. Vander, Wagner, G., Wilzewski, J., Wcisło, P., Yu, S., and Zak, E.J. (2017). The HITRAN2016 molecular spectroscopic database. J. Quant. Spectrosc. Radiat. Transf., 203:3-69.

Grauer, S.J., Conrad, B.M., Miguel, R.B., and Daun, K.J. (2018). Gaussian Model for Emission Rate Measurement of a Heated Plume using Hyperspectral Data. J. Quant. Spectrosc. Radiat. Transf., 206:125-134.

Hanson, R.K., Spearrin, R.M., and Goldenstein, C.S. (2016). Spectroscopy and Optical Diagnostics for Gases, 1st ed. Springer International Publishing, Cham.

Hart, A. (2001). Mann-Whitney test is not just a test of medians: differences in spread can be important. BMJ, 323:391-393.

Herschel Space Observatory (2013). PACS Observer's Manual (HERSCHEL-HSCDOC-0832, V. 2.5.1).

Höök, M., Davidsson, S., Johansson, S., and Tang, X. (2014). Decline and depletion rates of oil production: A comprehensive investigation. Philos. Trans. R. Soc. A Math. Phys. Eng. Sci., 372(2006).

Howell, J.R., Siegel, R., and Mengüç, M.P. (2016). Thermal Radiation Heat Transfer, 6th ed. CRC Press, Boca Raton, FL.

Jefferson, A.M. (2017). Exploratory Experiments to Determine Effects of Injected Aerosolized Water, Hydrochloric Acid, and Sodium Chloride Solutions on LabScale Flare Emissions. Carleton University, Ottawa, ON.

Johnson, M.R., Devillers, R.W., and Thomson, K.A. (2013). A generalized sky-LOSA method to quantify soot/black carbon emission rates in atmospheric plumes of gas flares. Aerosol Sci. Technol., 47(9):1017-1029.

Johnson, M.R. and Kostiuk, L.W. (2002). A parametric model for the efficiency of a flare in crosswind. Proc. Combust. Inst., 29(2):1943-1950.

Johnson, M.R. and Kostiuk, L.W. (2000). Efficiencies of low-momentum jet diffusion flames in crosswinds. Combust. Flame, 123(1-2):189-200.

Johnson, M.R., Wilson, D.J., and Kostiuk, L.W. (2001). A fuel stripping mechanism for wake-stabilized jet diffusion flames in crossflow. Combust. Sci. Technol., 169(1):155-174. 
Johnston, R., Jones, K., and Manley, D. (2018). Confounding and collinearity in regression analysis: a cautionary tale and an alternative procedure, illustrated by studies of British voting behaviour. Qual. Quant., 52(4):1957-1976.

Kaye, W. (1997). Emission Spectra of Argon, Helium, Krypton, Neon and Xenon PENRAY Lamps.

Kazemimanesh, M., Kuang, C., Kostiuk, L.W., and Olfert, J.S. (2020). Effect of sodium chloride on the evolution of size, mixing state, and light absorption of soot particles from a smoking laminar diffusion flame. Combust. Flame, 218:168-178.

Knighton, W.B., Herndon, S.C., Franklin, J.F., Wood, E.C., Wormhoudt, J., Brooks, W., Fortner, E.C., and Allen, D.T. (2012). Direct measurement of volatile organic compound emissions from industrial flares using real-time online techniques: Proton Transfer Reaction Mass Spectrometry and Tunable Infrared Laser Differential Absorption Spectroscopy. Ind. Eng. Chem. Res., 51(39):12674-12684.

Kramida, A., Ralchenko, Y., Reader, J., and NIST ASD Team (2020). NIST: Atomic Spectra Database (version 5.8). Available at https://physics.nist.gov/PhysRefData/ASD/Html/verhist.shtml (Accessed 9 April 2019).

Lajunen, L.H.J. (2004). Spectrochemical Analysis by Atomic Absorption and Emission, 2nd ed. The Royal Society of Chemistry.

Lewis, R.J. (2004). Sax's Dangerous Properties of Industrial Materials, 11th ed, Choice Reviews Online. John Wiley \& Sons, Inc., Hoboken, NJ, USA.

Lou, C., Chen, C., Sun, Y., and Zhou, H. (2010). Review of soot measurement in hydrocarbon-air flames. Sci. China Technol. Sci., 53(8):2129-2141.

Macheret, S.O., Losev, S.A., Chernyi, G.G., and Potapkin, B. V. (2002). Physical and Chemical Processes in Gas Dynamics. American Institute of Aeronautics and Astronautics, Reston, VA.

MacIsaac, D., Kanner, G., and Anderson, G. (1999). Basic physics of the incandescent lamp (lightbulb). Phys. Teach., 37(9):520-525.

Macskassy, S. a, Provost, F., Rosset, S., and Heights, Y. (2005). Pointwise ROC Confidence Bounds: An Empirical Evaluation., in The Twenty-Second International Conference on Machine Learning (ICLM), Bonn, Germany.

Maguire-Boyle, S.J. and Barron, A.R. (2014). (SI) Organic compounds in produced waters from shale gas wells. Environ. Sci. Process. Impacts, 16(10):2237-48.

Majnik, M. and Bosnić, Z. (2013). ROC analysis of classifiers in machine learning: A survey. Intell. Data Anal., 17(3):531-558.

Martinez, W. and Martinez, A. (2007). Computational Statistics Handbook with MATLAB, Second Edition (Computer Science and Data Analysis). 


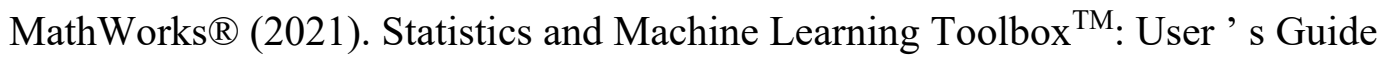
(R2012a).

Mekhrengin, M., Miroshnichenko, G., Chistiakov, A., Bolotov, D., Ashirov, A., Zubko, A., and Meshkovskiy, I. (2020). Combination of soot pyrometry and C2* emission spectroscopy for temperature measurement during combustion of hydrocarbons. Meas. J. Int. Meas. Confed., 166:108242.

Mekhrengin, M. V., Meshkovskii, I.K., Tashkinov, V.A., Guryev, V.I., Sukhinets, A. V., and Smirnov, D.S. (2019). Multispectral pyrometer for high temperature measurements inside combustion chamber of gas turbine engines. Meas. J. Int. Meas. Confed., 139:355-360.

Menard, S. (2002). Applied Logistic Regression Analysis, Serise: Quantitative applications in the Social Sciences. Sage Publications Inc., Thousand Oaks, CA.

Midi, H., Sarkar, S.K., and Rana, S. (2010). Collinearity diagnostics of binary logistic regression model. J. Interdiscip. Math., 13(3):253-267.

Modest, M.F. (2013). Radiative Heat Transfer, Third Edit. ed. Elsevier Inc., Oxford, UK.

Mokhatab, S., Poe, W.A., and Mak, J.Y. (2018). Handbook of natural gas transmission and processing: Principles and practices, Fourth. ed, Handbook of Natural Gas Transmission and Processing: Principles and Practices. Gulf Professional Publishing.

Motulsky, H. and Christopoulos, A. (2003). Fitting Models to Biological Data using Linear and Nonlinear Regression. Fitting Model. to Biol. Data Using Linear Nonlinear Regres., 296-297.

Motulsky, H.J. (2021). GraphPad Statistics Guide. Available at http://www.graphpad.com/guides/prism/7/statistics/index.htm

Navidi, W. (2010). Statistics for engineers and scientists, 3rd. ed. ed. McGraw-Hill.

North Dakota Industrial Commision Oil and Gas Division (2021). Well Index. Available at https://www.dmr.nd.gov/oilgas/feeservices/flatfiles/flatfiles.asp (Accessed 15 April 2021).

Potts, P.J. (1992). A Handbook of Silicate Rock Analysis. Springer US, Boston, MA.

Provost, F., Fawcett, T., and Kohavi, R. (1997). The Case Against Accuracy Estimation for Comparing Induction Algorithms., in Fifteenth International Conference on Machine Learning, San Francisco, CA.

Roth, C., Milani, Z.R., and Johnson, M.R. (2020). Emissions of Lab-Scale Flares with Entrained Salt Aerosols., in AGU Fall Meeting, San Francisco, CA.

Scheeline, A. (2017). How to Design a Spectrometer. Appl. Spectrosc., 71(10):22372252. 
Schnaiter, M., Horvath, H., Mohler, O., Naumann, K., Saathoff, H., and Schock, O. (2003). UV-VIS-NIR spectral optical properties of soot and soot-containing aerosols. J. Aerosol Sci., 34(10):1421-1444.

Shaban, H.I. (1995). A study of foaming and carry-over problems in oil and gas separators. Gas Sep. Purif., 9(2):81-86.

Snelling, D.R., Smallwood, G.J., Liu, F., Gülder, Ö.L., and Bachalo, W.D. (2005). A calibration-independent laser-induced incandescence technique for soot measurement by detecting absolute light intensity. Appl. Opt., 44(31):6773-6785.

Stanton, J.S., Anning, D.W., Brown, C.J., Moore, R.B., McGuire, V.L., Qi, S.L., Harris, A.C., Dennehy, K.F., McMahon, P.B., Degnan, J.R., and Böhlke, J.K. (2017). Brackish groundwater in the United States: U.S. Geological Survey Professional Paper 1833.

Stohl, A., Klimont, Z., Eckhardt, S., Kupiainen, K., Shevchenko, V.P., Kopeikin, V.M., and Novigatsky, A.N. (2013). Black carbon in the Arctic: the underestimated role of gas flaring and residential combustion emissions. Atmos. Chem. Phys., 13(17):88338855 .

Strosher, M.T. (2000). Characterization of emissions from diffusion flare systems J. Air Waste Manage. Assoc.,.

Torres, V.M., Herndon, S.C., Wood, E., Al-Fadhli, F.M., and Allen, D.T. (2012). Emissions of Nitrogen Oxides from Flares Operating at Low Flow Conditions. Ind. Eng. Chem. Res., 51(39):12600-12605.

Trivanovic, U., Baldelli, A., Kazemimanesh, M., Conrad, B.M., Jefferson, A.M., Corbin, J.C., Johnson, M.R., Olfert, J.S., and Rogak, S.N. (2019). The Effect of Inorganic Salts from Flowback Operations on the Size, Effective Density, Mixing State, and Optical Properties of Soot from Gas Flares., in Combustion Institute Canadian Section Spring Technical Meeting, Kelowna, BC, p. 6.

U.S. EIA (2019). Natural Gas Gross Withdrawls and Production: Vented and Flared. Available at https://www.eia.gov/dnav/ng/ng_prod_sum_a_EPG0_VGV_mmcf_a.htm

U.S. EPA (2021). Critera Air Pollutants. Available at https://www.epa.gov/criteria-airpollutants

U.S. EPA and OAQPS (2012). Parameters for Properly Designed and Operated Flares. United States Environmental Protection Agency (U.S. EPA) Office of Air Quality Planning and Standard (OAQPS).

van de Hulst, H.C. (1957). Light Scattering by Small Particles. Dover publications.

Veil, J. (2020). U.S. Produced Water Volumes and Management Practices in 2017. 
Voxman, W.L. and Goetschel Jr., R.H. (1981). Advanced Calculus: An Introduction to Modern Analysis. Marcel Dekker, New York, NY.

Weisstein, E.W. (2021). Gaussian Function. Available at https://mathworld.wolfram.com/GaussianFunction.html

Wimberly, B.J. (2015). Strontium Monoxide Measurements in Methane- air Flames. University of Tennessee - Knoxville.

Wines, T.H. and Brown, R.L. (1994). Scientific \& Technical Report: Recent Developments In Liquid / Gas Separation Technology., in Laurance Reid Gas Conditioning Conference, Pall Corporation, Norman.

Witte, M.J. (2015). Diatomic Carbon Measurements with Laser- Induced Breakdown Spectroscopy.

World Bank (2020a). An Overview of the Global Gas Flaring Reduction Partnership (GGFR). Available at https://www.worldbank.org/en/programs/gasflaringreduction\#1

World Bank (2020b). Global Gas Flaring Tracker Report, Global Gas Flaring Reduction Partnership. The World Bank. 


\section{Appendix A: Supplemental Derivations and Equations for the Atomic Emission, Molecular Absorption, and Soot Temperature Models}

This appendix explains the derivation of the forms of the radiative emission models for soot, atomic emission of $\mathrm{Na}$ or $\mathrm{K}$, and absorption by $\mathrm{O}_{2}$ that were used in this thesis. The derivation of the radiative emission models for soot were originally developed by Dr. Bradley Conrad, a post doctoral researcher in the Energy \& Emissions Research Lab at Carleton University.

\section{A.1 The Radiative Transfer Equation Derivation for Soot Emission from a Flare Flame Measured by the Spectrometer System}

Consider an optical axis transecting a flame and assume that ambient light is negligible in intensity relative to emitted light from soot in the flame. The measured spectral intensity of soot emission at the location of the spectrometer system $\left(I(\tilde{v})\left[\mathrm{W} \mathrm{m}^{-2} \mathrm{sr}^{-1}\left(\mathrm{~cm}^{-1}\right)^{-1}\right]\right)$, for an optical axis traversing a flame from 0 to $x$ can be determined using the simplified radiative transfer equation (RTE) (adapted from e.g., Howell et al. 2016; Modest 2013):

$$
I(\tilde{v})=\int_{0}^{x} I_{B B-P}\left(\tilde{v}, T\left(x^{\prime}\right)\right) \kappa_{a}\left(\tilde{v}, x^{\prime}\right) e^{-\int_{x^{\prime}}^{x} \kappa_{e}\left(\widetilde{v}, x^{\prime \prime}\right) d x^{\prime \prime}} d x^{\prime}
$$

where $I_{B B-P}(\tilde{v}, T)$ is the spectral blackbody intensity $\left[\mathrm{W} \mathrm{m}^{-2} \mathrm{sr}^{-1}\left(\mathrm{~cm}^{-1}\right)^{-1}\right]$ for temperature $T[\mathrm{~K}]$ (i.e., Planck's blackbody equation), and $\kappa_{a}$ and $\kappa_{e}$ are the spectral absorption and extinction coefficients $\left[\mathrm{m}^{-1}\right]$ that generally vary with $x$ due to variations in temperature and composition of optically active species in the spectrum of interest.

Light that is emitted from the flare flame and measured by the spectrometer system is dominated by soot emission. Introducing the spectral absorption and extinction crosssections of soot $\left(\sigma_{a}(\tilde{v})\right.$ and $\left.\sigma_{e}(\tilde{v})\left[\mathrm{m}^{2}\right]\right)$ - which, when multiplied with the local number density of soot $\left(n(x)\right.$ [particles $\left.\left.\mathrm{m}^{-3}\right]\right)$, yield the spectral absorption and extinction coefficients - gives: 


$$
I(\tilde{v})=\int_{0}^{x} I_{B B-P}\left(\tilde{v}, T\left(x^{\prime}\right)\right) \sigma_{a}(\tilde{v}) n\left(x^{\prime}\right) e^{-\int_{x^{\prime}}^{x} \sigma_{e}(\widetilde{v}) n\left(x^{\prime \prime}\right) d x^{\prime \prime}} d x^{\prime}
$$

Introducing spectral co-albedo of soot, which is the ratio of the spectral absorption-toextinction cross-sections $\left(\widetilde{\omega}(\tilde{v})=\sigma_{a}(\tilde{v}) / \sigma_{e}(\widetilde{v})[-]\right)$, the RTE can be re-formulated as:

$$
I(\tilde{v})=\widetilde{\omega}(\tilde{v}) \int_{0}^{x} I_{B B-P}\left(\tilde{v}, T\left(x^{\prime}\right)\right) \sigma_{e}(\tilde{v}) n\left(x^{\prime}\right) e^{-\int_{x^{\prime}}^{x} \sigma_{e}(\widetilde{v}) n\left(x^{\prime \prime}\right) d x^{\prime \prime}} d x^{\prime}
$$

Leveraging mean value theorem (e.g., Voxman and Goetschel Jr. 1981), the RTE can be further simplified by defining an effective temperature $\left(T_{g}\right)$ and removing the blackbody intensity from the integral, giving:

$$
I(\tilde{v})=I_{B B-P}\left(\tilde{v}, T_{g}\right) \widetilde{\omega}(\widetilde{v}) \int_{0}^{x} \sigma_{e}(\tilde{v}) n\left(x^{\prime}\right) e^{-\int_{x^{\prime}}^{x} \sigma_{e}(\widetilde{v}) n\left(x^{\prime \prime}\right) d x^{\prime \prime}} d x^{\prime}
$$

Using the first fundamental theorem of calculus, the remaining integral can be simplified via (e.g., Conrad et al. 2020; Grauer et al. 2018):

$$
\begin{aligned}
\int_{0}^{x} \sigma_{e}(\tilde{v}) n\left(x^{\prime}\right) e^{-\int_{x^{x}}^{x} \sigma_{e}(\tilde{v}) n\left(x^{\prime \prime}\right) d x^{\prime \prime}} d x^{\prime} & =1-e^{-\int_{0}^{x} \sigma_{e}(\tilde{v}) n\left(x^{\prime \prime}\right) d x^{\prime \prime}} \\
& =1-e^{-\sigma_{e}(\tilde{v}) \bar{n} L}
\end{aligned}
$$

where $\bar{n}$ is the path-averaged number density of soot [particles $\mathrm{m}^{-3}$ ] and $L$ is the path length through the flame [m]. Substituting Eq. (A.5) this into Eq.(A.4), and re-introducing the absorption cross-section gives a simplified form of the RTE:

$$
I(\tilde{v})=I_{B B-P}\left(\tilde{v}, T_{g}\right) \widetilde{\omega}(\tilde{v})\left(1-e^{-\frac{\sigma_{a}(\tilde{\mathcal{v}})}{\tilde{\omega}(\tilde{n})} \bar{n}}\right)
$$

In the limiting case of sufficiently low temperature and high wavenumber, the exponential operator in the denominator of Planck's blackbody equation is much greater than unity. This permits simplification of the spectral blackbody intensity to Wien's Approximation (e.g., Howell et al. 2016):

$$
I(\widetilde{v})=I_{B B-W}\left(\tilde{v}, T_{g}\right) \widetilde{\omega}(\widetilde{v})\left(1-e^{-\frac{\sigma_{a}(\tilde{v})}{\tilde{\omega}(\tilde{v})} \bar{n} L}\right)
$$


which approximates Planck's blackbody equation to within 1\% for adiabatic flame temperatures typical of upstream oil and gas flares (i.e., $<2300[\mathrm{~K}]$ ) and the wavenumber ranges relevant to this study, 11111 to $25000 \mathrm{~cm}-1$ (or, 400 to $900 \mathrm{~nm}$ ) (Alkemade et al. 1982).

For flame-generated soot, spectral variability of absorption is characterized by a powerlaw using the "Absorption Ångström Exponent" ( $A A E)$, such that $\sigma_{a}(\tilde{v}) \approx \tilde{v}^{A A E}$ (Schnaiter et al. 2003). Introducing this into Eq. (A.7) gives:

$$
I(\tilde{v})=I_{B B-W}\left(\tilde{v}, T_{g}\right) \widetilde{\omega}(\tilde{v})\left(1-e^{-\frac{\widetilde{v}^{A A E}}{\widetilde{\omega}(\widetilde{v})} \bar{n} L}\right)
$$

such that typical literature values for the $A A E$ of over-flame soot with an assumed $10 \%$ standard deviation may be defined by: $A A E \sim \mathrm{N}(1,0.1)$ (van de Hulst 1957).

The spectral variability of the co-albedo is not well-discussed in the literature; however, optical and morphological properties of soot from flare-like flames are well-defined (Johnson et al. 2013). Leveraging the published probability distributions for the fundamental properties of flare-generated soot (Johnson et al. 2013), the spectral co-albedo ratio generally follows an exponential function such that $\widetilde{\omega}(\tilde{v}) \approx e^{B \tilde{v}}$. Introducing this into Eq. (A.8) gives:

$$
I(\tilde{v})=I_{B B-W}\left(\tilde{v}, T_{g}\right) e^{B \tilde{v}}\left(1-e^{-\frac{\widetilde{v} A A E}{e^{B \tilde{v}}} \bar{n} L}\right)
$$

such that $B[-]$ is a constant characterizing the spectral variability in the fraction of soot extinction due to absorption. The distribution of $B$ was estimated by a Monte-Carlo framework that computed the co-albedo of soot assuming that the refractive index of soot was spectrally constant, and by using soot properties from Johnson et al. (2013) such that: $B \sim \mathrm{N}\left(-1.585 \times 10^{-5}[\mathrm{~cm}], 4.161 \times 10^{-6}[\mathrm{~cm}]\right)$.

Eq. (A.9) can be reduced to two equations that model the flare as optically thin (OTN) or thick (OTK), representing the lower and upper limit bounds of $L$. This is important because the values of $T, L$, and $\bar{n}$ are unknown, meaning that to solve for any one of them via least squares regression would require solving for all of them simultaneously. Applying the 
OTN assumption to Eq. (A.9) brings $L$ and $\bar{n}$ outside of the exponent such that they can then be eliminated by normalization, whereas applying the OTK assumption to Eq. (A.9) directly eliminates $L$ and $\bar{n}$. The actual $T_{g}$ of soot emission that is measured by the spectrometer system is assumed to lay in between $T_{\text {OTN }}$ and $T_{\text {OTK }}$.

As described above, the OTN limit $(L \rightarrow 0)$ leverages the assumption:

$$
e^{-\frac{\widetilde{v}^{A A E}}{e^{B \tilde{v}}} \bar{n} L} \approx 1-\frac{\tilde{v}^{A A E}}{e^{B \tilde{v}}} \bar{n} L
$$

where the spectral radiance of soot emission from an OTN flame incident to the linear detector of the spectrometer system is:

$$
I_{\text {OTN }}(\tilde{v})=I_{B B-W}\left(\tilde{v}, T_{O T N}\right)\left(\tilde{v}^{A A E} \bar{n} L\right)
$$

whereas the OTK limit $(L \rightarrow \infty)$ leverages this assumption:

$$
e^{-\frac{\widetilde{v}^{A A E}}{e^{B \widetilde{v}}} \bar{n} L} \approx 0
$$

where the spectral radiance of soot emission from an OTK flame incident to the linear detector of the spectrometer system is:

$$
I_{O T K}(\tilde{v})=I_{B B-W}\left(\tilde{v}, T_{O T K}\right) e^{B \tilde{v}}
$$

To isolate for $T_{O T N}$ and $T_{O T K}$ in Eq. (A.11) and (A.13), they were first normalized against some reference wavenumber $\tilde{v}_{o}$ :

$$
\begin{gathered}
\frac{I_{\text {OTN }}(\tilde{v})}{I_{\text {OTN }}\left(\tilde{v}_{O}\right)}=\frac{I_{B B-W}\left(\tilde{v}, T_{\text {OTN }}\right)}{I_{B B-W}\left(\tilde{v}_{O}, T_{\text {OTN }}\right)}\left(\frac{\tilde{v}}{\tilde{v}_{O}}\right)^{A A E} \\
\frac{I_{\text {OTK }}(\tilde{v})}{I_{\text {OTK }}\left(\tilde{v}_{O}\right)}=\frac{I_{B B-W}\left(\tilde{v}, T_{O T K}\right)}{I_{B B-W}\left(\tilde{v}_{O}, T_{O T K}\right)}\left(e^{-B \tilde{v}_{o}(1-\tilde{v})}\right)
\end{gathered}
$$

The ratio of Wien's Approximation in Eq. (A.14) and (A.15) may be, in general, reduced according to:

$$
\frac{I_{B B-W}(\tilde{v}, T)}{I_{B B-W}\left(\tilde{v}_{o}, T\right)}=\left(\frac{\tilde{v}}{\tilde{v}_{o}}\right)^{3} e^{\left(\frac{h c \widetilde{v}_{o}}{k_{B} T}\left(1-\frac{\widetilde{v}}{\tilde{v}_{O}}\right)\right)}
$$

Substituting Eq. (A.16) in Eq. (A.14) and (A.15) leads to the general form of soot emission from an OTN and OTK flame was: 


$$
\frac{I(\tilde{v})}{I\left(\tilde{v}_{o}\right)}=\left(\frac{\tilde{v}}{\tilde{v}_{o}}\right)^{\zeta} e^{\left(\eta\left(1-\frac{\tilde{v}}{\tilde{v}_{o}}\right)\right)}
$$

where, for an OTN flame $(\zeta, \eta, T)=\left([3+A A E],\left[\frac{h c \tilde{v}_{o}}{k_{B} T}\right], T_{\text {OTN }}\right)$ and for an OTN flame $(\zeta, \eta, T)=\left(3,\left[\frac{h c \tilde{v}_{o}}{k_{B} T}-B \tilde{v}_{o}\right], T_{O T K}\right)$.

\section{A.2 The Gaussian Function with Standard Deviation Replaced by Full-Width Half-Max}

The functional form of the Gaussian kernel that is used to describe the spectral broadening caused by the dispersion of light onto the spectrometer's linear detector (i.e., the instrument profile) is (the general Gaussian function is from Weisstein (2021):

$$
\Phi(\tilde{v}, \gamma)=A(\gamma) e^{-4 \ln 2 \frac{\left(\widetilde{v}-\widetilde{v}_{\left.o, i_{j}\right)^{2}}\right.}{\gamma^{2}}}
$$

where standard deviation $(\sigma[-])$ was replaced by full-width half-max (FWHM, $\gamma[-])$ via the relationship, $\sigma=\frac{\gamma}{2 \sqrt{2 \ln 2}}$, and $A$ is the normalized height of the Gaussian profile [-] (Weisstein 2021):

$$
A=\frac{2 \sqrt{2 \ln 2}}{\gamma \sqrt{2 \pi}}
$$

\section{A.3 The Spectral Absorption Coefficient Function used to Model Absorption and Emission Lines}

The functional form of the spectral absorption coefficient used to model absorption and emission lines is described by Goldenstein et al. (2017):

$$
k_{i_{j}}=\sum_{j} S_{j} n x_{i} \phi_{j}
$$

where $S_{i_{j}}$ is linestrength $\left.\left[\mathrm{cm}^{3} \text { (atom or molecule) }\right)^{-1}\right]$ of a atomic or molecular line (for functional form see Goldenstein et al. (2017)), $n$ is the number density of the gas (e.g., number density of the flame or ambient air in-between flare flame and spectrometer system), $x_{i}$ is the volume fraction of the given species, and $\phi_{j}$ is the lineshape function 
which models spectral broadening $[\mathrm{cm}]$ that occurs during the radiative process. Goldenstein et al. (2017) describes $\phi$ as having a Voigt profile which is a convolution of Lorentzian and Gaussian profiles. Respectively, these profiles describe the Doppler and collisional broadening that occurs during the line's radiative process (Goldenstein et al. 2017). 


\section{Appendix B: Age Distribution of Active Oil and Gas Wells in North Dakota}

Figure B.1 shows the age distribution of active oil and gas wells in North Dakota during the field measurement campaign in North Dakota in November 2019. These data were extracted from the publicly accessible "Well Index" database maintained by the North Dakota Industrial Commission Oil and Gas Division (North Dakota Industrial Commision Oil and Gas Division 2021). For the purpose of this figure, well age was calculated for each well by subtracting the reported date of its initial production test from the first day of the measurement campaign, November $8^{\text {th }}, 2019$.

Both the well population (orange histogram) and the wells linked to the flares measured in this thesis (blue histogram) have bimodal shapes that align closely. However, $\sim 11 \%$ of the wells in North Dakota are $>11$ years old, none of which were linked to any flare measured for this study. Results from Levene and Mann-Whitney tests suggest that the age of wells linked to measured flares tends to be statistically younger than the general well population. Importantly, since not all wells would be tied to flares, it is unclear whether this difference means there would be a corresponding difference between ages of wells in the present sample and ages of all wells tied to flares in North Dakota. If there is a difference, it is possible that there may have been some unintended influence in the way flares were selected for measurement in the field, as larger more readily identifiable flares at more accessible locations were favoured. Since larger flames are typically the result of higher gas production volumes, this may also correlate with younger wells. That said, even if flare selection was partial to younger wells, neither well age nor production volume data were determined to be good predictors of liquid carry-over meaning that several other factors are likely relevant.

Some other factors that might influence liquid carry-over include the design and sizing of the separator system, the upkeep and operational practices of the separator, or even weather at the time of measurement since the separation process may be sensitive to temperature and downstream pressure. Moreover, since flare volumes are almost certainly correlated 
with hydrocarbon production volumes which tend to decline with well age, it is arguably more important to sample flares with younger wells ( $<11$ years old) since they would likely be responsible for the majority of flare emissions.

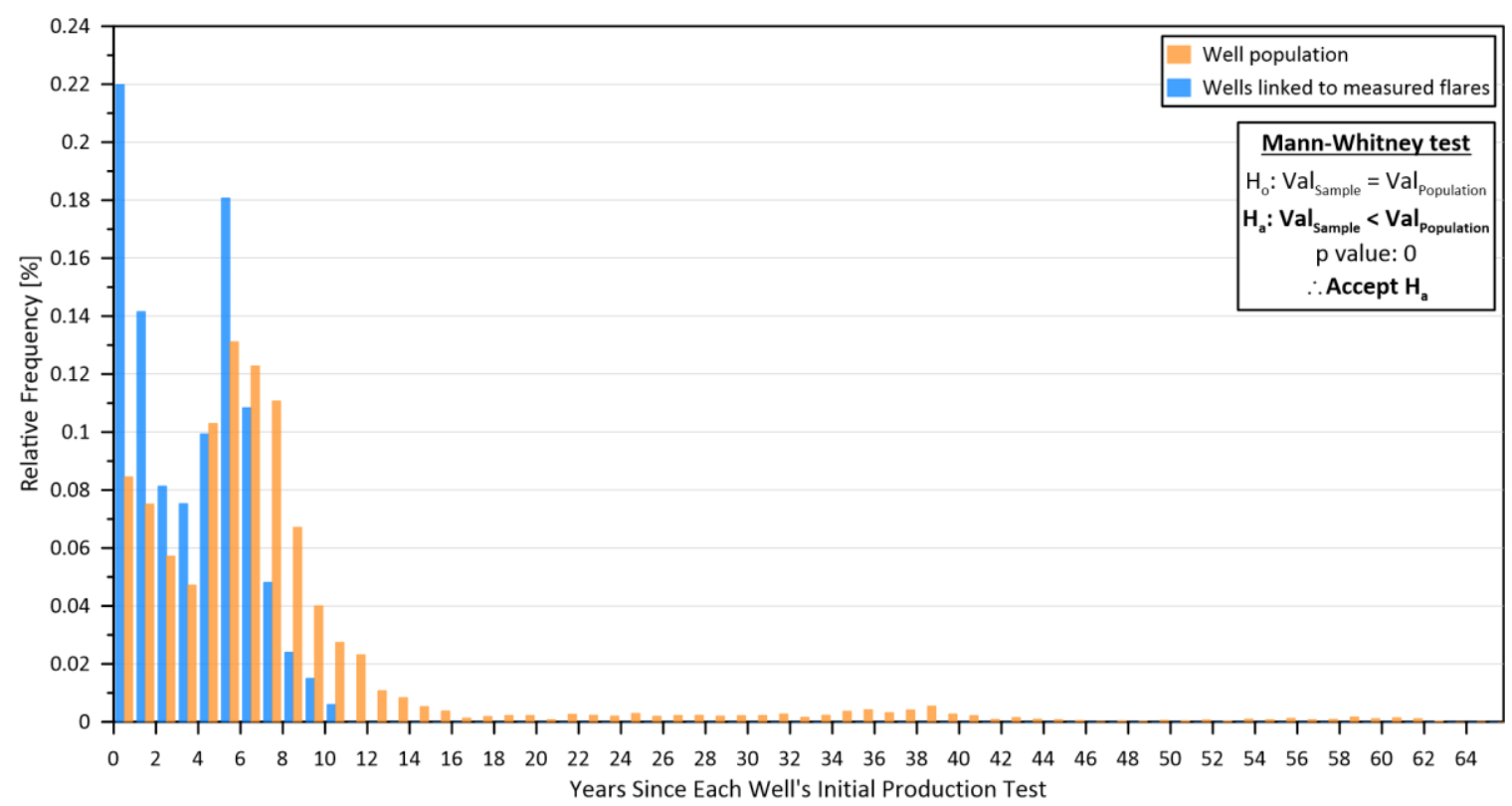

Figure B.1: The age distributions of all active oil and gas well in North Dakota on November $8^{\text {th }}, 2019$ and the subset of wells that were linked to measured flares. 